\title{
MESSENGER observations of Mercury's nightside magnetosphere under extreme solar wind conditions: reconnection-generated structures and steady convection
}

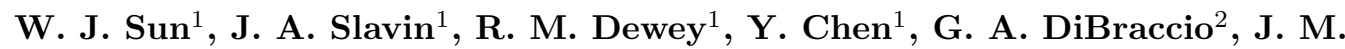 \\ Raines $^{1}$, J. M. Jasinski ${ }^{3}$, X. Jia ${ }^{1}$, M. Akhavan-Tafti ${ }^{1}$ \\ ${ }^{1}$ Department of Climate and Space Sciences and Engineering, University of Michigan, Ann Arbor, \\ Michigan 48109, USA. \\ ${ }^{2}$ Solar System Exploration Division, NASA Goddard Space Flight Center, Greenbelt, Maryland, USA. \\ ${ }^{3}$ NASA Jet Propulsion Laboratory, California Institute of Technology, California, USA.
}

\section{Key Points:}

- Coronal mass ejections drive more intense nightside reconnection than high speed streams

- Under extreme conditions, magnetic reconnection produces a distorted Hall magnetic field pattern in the plasma sheet.

- Continued intense solar wind forcing does not produce substorm magnetic flux loading and unloading of tail lobe instead steady convection

This is the author manuscript accepted for publication and has undergone full peer review but has not been through the copyediting, typesetting, pagination and proofreading process, which

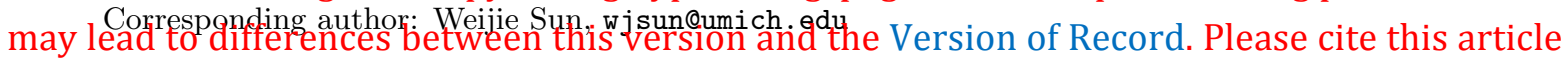
as doi: 10.1029/2019JA027490 


\begin{abstract}
Mercury's nightside magnetosphere is investigated under the impact of a coronal mass ejection (CME) and a high-speed stream (HSS) with MESSENGER observations. The CME was shown to produce a low plasma $\beta$ (ratio of thermal pressure to magnetic pressure) magnetosheath, while the HSS creates a higher $\beta$ magnetosheath. Reconnection at the dayside magnetopause was found to be stronger during the CME than the HSS but both were stronger than the average condition (Slavin et al., 2014, doi:10.1002/2014JA020319). Here we show that the CME and HSS events produced large number of flux ropes and dipolarization fronts in the plasma sheet, respectively. The occurrence rates for the structures were approximately two orders of magnitude higher than under average conditions with the rates during CME's being twice that of HSS's. The flux ropes appeared as quasiperiodic flux rope groups. Each group lasted approximately one minute, and had few large flux ropes followed by several smaller flux ropes. The lobe magnetic flux accounted for around half of the Mercury's available magnetic flux with the flux during CME's being larger than that of HSS's. The CME produced a more dynamic nightside magnetosphere than the HSS. Further, for the CME event, the tail magnetic reconnection produced a distorted Hall magnetic field pattern and the X-line had a dawn-dusk extent of $20 \%$ of the tail width. No magnetic flux loading and unloading events were observed suggesting that, during these intense driving conditions, Mercury's magnetosphere responded with a type of quasi-steady convection as opposed to the tail flux loading-unloading events seen at Earth.
\end{abstract}

\title{
1 Introduction
}

Mercury is the smallest and the innermost planet in the Solar System with an aphelion of $\sim 0.47 \mathrm{AU}$ and a perihelion of $\sim 0.31 \mathrm{AU}$, in which $\mathrm{AU}$ is the distance from Earth to the Sun. Three Mercury's flybys by Mariner 10 in the 1970s discovered the planet's intrinsic magnetic field (Ness et al., 1974), which is in the same magnetic polarity as that of Earth's but is much weaker in magnitude. Later studies, especially those based on measurements from MErcury Surface, Space ENviroment, GEochemistry, and Ranging (MESSENGER) (Solomon et al., 2001), show that the intrinsic magnetic field is highly dipole and closely aligns $\left(<0.8^{\circ}\right)$ with the planet's rotation axis. The magnetic equator has a northward offset of $\sim 0.2 R_{M}$ (where $R_{M}$ is Mercury's mean radius, one $R_{M}$ is $\sim 2440 \mathrm{~km}$ ) and the dipole moment is $\sim 190 n T \cdot R_{M}^{3}$ (Alexeev et al., 2008; Anderson et al., 2010, 2012). In Mercury's orbit, strong interplanetary magnetic field (IMF) intensity and high solar wind density result in low Alfvén Mach number (e.g., Russell et al., 1988; Sarantos \& Slavin, 2009), which favors thick plasma depletion layers to form in front of the dayside magnetopause and lead to high dayside dimensionless reconnection rate $(\sim 0.15)$ (Scurry et al., 1994; DiBraccio et al., 2013; Gershman et al., 2013; Slavin et al., 2009, 2014). The small dipole moment and the strong solar wind dynamic pressure make the planet occupies a large portion of the magnetosphere with the average standoff distance from the dipole center to the subsolar magnetopause of $\sim 1.5 R_{M}$ (Ness et al., 1976; Slavin et al., 2010; Winslow et al., 2013).

Magnetic reconnection at the dayside magnetopause (e.g., Mercury and Earth) creates open field lines with one end connecting to the planets and the other end to the IMF. The open field lines enable shocked solar wind plasma to enter magnetospheres. As the open field lines convect anti-sunward, plasma populations in the open flux tubes connecting the cusp may precipitate or mirror away and then transport to nightside lobes and form plasma mantle. There are observations on the plasma mantle in Earth's magnetosphere (Rosenbauer et al., 1975; Sckopke et al., 1976) and Mercury's magnetosphere (DiBraccio, Slavin, Raines, et al., 2015; Jasinski et al., 2017). The open field lines in the lobes convect toward the magnetic equatorial plane and are closed by magnetic reconnection in the cross-tail current sheet, and then convect to the dayside magnetosphere where it can be opened again through the dayside magnetopause reconnection. The cir- 
culation of plasma, magnetic flux and energy in this process constitutes the Dungey cycle (Dungey, 1961).

In Earth's magnetosphere, the solar wind-magnetosphere-ionosphere coupling produces several magnetospheric modes. The magnetospheric modes include substorms (e.g., Akasofu, 1964; Baker et al., 1996; McPherron et al., 1973), steady magnetospheric convection events (SMCs) (e.g., Pytte et al., 1978; Sergeev et al., 1996), and sawtooth oscillations (e.g., Belian et al., 1995). During the substorms, magnetic flux loads into the tail lobes and then unloads through magnetotail reconnection, which corresponds to the magnetic flux loading-unloading. Substorms normally persist $\sim 1$ to 3 hours. In the SMCs, magnetic reconnection continue to occur in the magnetotail, but magnetic field intensity in the lobes remain stable. SMCs often last a period of several substorms $(>5$ to 10 hours). In the SMC, flux transfer rates in and out of the magnetotail should be equal, which also termed as continuous magnetospheric dissipation (CMD) (Tanskanen et al., 2005). The sawtooth oscillations are consisted of quasi-periodic Dungey cycles with magnetic flux amplitude in the tail lobes much stronger than that of isolated substorms (Huang et al., 2003; Henderson et al., 2006). Isolated substorms refer to substorms without neighboring substorms within few hours. Solar wind drivers are distinct for the magnetospheric modes at Earth. SMC events and sawtooth oscillations require solar wind speed and IMF southward $B_{z}$ to be steady in a period of several substorms, and the intensity of the drivers of sawtooth oscillations are stronger than those of SMC events. However, isolated substorms do not require steadiness and intensity of the drivers (O'Brien et al., 2002; DeJong et al., 2009; Pulkkinen et al., 2007; Partamies et al., 2009). On the other hand, several studies show that the ionosphere could play a role in the sawtooth oscillations (Brambles et al., 2011; Zhang et al., 2016).

Magnetic reconnection-related structures, including magnetic flux ropes and dipolarization fronts, are often observed in Mercury's magnetotail (Slavin et al., 2009; Slavin, Anderson, et al., 2012; DiBraccio, Slavin, Imber, et al., 2015; Sun et al., 2016, 2018; Dewey et al., 2017, 2018; Zhao et al., 2019). The occurrence rates of the reconnection-related structures are around an order of magnitude higher at Mercury than at Earth implying a more dynamic plasma sheet in Mercury's magnetotail (Sun et al., 2016; Smith et al., $2017,2018)$. The reconnection-related structures are more frequently observed on the dawnside plasma sheet than on the duskside (Sun et al., 2016, 2017; Smith et al., 2017), which is different to the duskside prominent reconnection features in Earth's plasma sheet (e.g., Nagai et al., 1998; Slavin et al., 2005; Imber et al., 2011). Recent three dimensional PIC simulations suggest that the short dawn-dusk extent of Mercury's magnetotail accounts for the difference in the dawn-dusk distributions of the magnetotail reconnection between the two planetary magnetospheres (Y.-H. Liu et al., 2019; Y. Chen et al., n.d.). In a possible encounter of magnetic reconnection diffusion region in Mercury's magnetotail, Zhong et al. (2018) reported a dimensionless reconnection rate of $\sim 0.2$ from the magnetic field measurements. In recent Magnetospheric Multiscale (MMS) studies in the Earth's cross-tail current sheet, dimensionaless reconnection rates range from $\sim 0.1$ to 0.2 (Genestreti et al., 2018; Nakamura et al., 2018).

In the studies by Slavin et al. (2014) and Jia et al. (2019), the authors investigated the characteristics of dayside magnetosphere under impacts of solar wind extreme events, including thick plasma depletion layer, low-altitude subsolar magnetopause and induction currents in Mercury's interior. Slavin et al. (2014) investigated three events, which are two Coronal Mass Ejection (CME) on 23 November 2011 and on 8 May 2012 and one high-speed stream (HSS) on 11 May 2012. The inferred solar wind pressures for the three events are from 45 to $60 \mathrm{nPa}$, and the distance of the subsolar magnetopause location is reduced from $\sim 1000 \mathrm{~km}$ to $\sim 100 \mathrm{~km}$ above the planet's surface. In these extreme solar wind dynamic pressure events, the reconnection rate on the dayside magnetopause is higher than the average condition. Therefore, more magnetic flux would be transferred from dayside to the nightside. 
In this study, we analyze the response of Mercury's nightside magnetosphere to two extreme solar wind events. Both extreme events are from Slavin et al. (2014). We focus on the CME on 23 November 2011 and the HSS on 11 May 2012, whose trajectories are close to the noon-midnight meridian. The trajectory of MESSENGER during the CME on 8 May 2012 in Slavin et al. (2014) deviated greatly from the noon-meridian plane towards the dawnside $\left(Y_{M S M}^{\prime}<-1.2 R_{M}\right)$, therefore, we ignored this event. The CME on 23 November 2011 produced a large number of tailward traveling flux ropes and the HSS on 11 May 2012 produced a large number of planetward traveling dipolarization fronts. Both types of structures occurred at occurrence rates approximately two orders of magnitude larger than the average occurrence rates for them in Mercury's magnetotail, implying the extremely dynamic plasma sheet. Open magnetic flux in the lobe corresponds to around half of Mercury's available magnetic flux confirming the extreme condition of Mercury's magnetosphere. However, the lobe magnetic field intensity was steady and lasted periods of several of Mercury's Dungey cycles, indicating that the magnetosphere experienced the quasi-steady convection. The low solar wind Alfvén Mach number and the absence of steady ring current and ionosphere at Mercury produce unique properties for steady convection events in Mercury's magnetosphere compared to Earth's magnetosphere.

\section{Overview of Extreme Nightside Magnetosphere}

\subsection{Data and Instrument}

The study utilizes particles and fields measurements from MESSENGER (Solomon et al., 2001). The ion measurements were provided by Fast Imaging Plasma Spectrometer (FIPS) (Andrews et al., 2007), which measures ions in an energy range from $\sim 50 \mathrm{eV} / \mathrm{q}$ to $\sim 13.3 \mathrm{keV} / q$ with an effective field of view of $\sim 1.15 \pi \mathrm{sr}$. The scan time of FIPS is $\sim 10$ seconds when inside of the magnetosphere, and is $\sim 1$ minute when outside of the magnetosphere. FIPS can distinguish ion species through the time-of-flight measurements. Magnetic field vectors were provided by the magnetometer (MAG) at a time resolution of 20 vectors per second (Anderson et al., 2007). In this study, the magnetic field data are shown in the Mercury solar magnetospheric coordinates (MSM) unless noted. In the MSM, the $\hat{x}_{M S M}$ is sunward, the $\hat{z}_{M S M}$ is northward and parallels to the dipole axis, and the $\hat{y}_{M S M}$ completes the right-handed coordinate system. The MSM coordinate shifts northward of $\sim 0.2 R_{M}$ from the center of Mercury due to the offset of the magnetic dipole (Anderson et al., 2010). Spacecraft position is provided with the same time resolution as the magnetic field data (20 samples per second), which is aberrated to be anti-parallel to the solar wind by rotating the $\hat{x}_{M S M}-\hat{y}_{M S M}$ plane.

During the CME on 23 November 2011 and the HSS on 11 May 2012, averaging over upstreams of outbound bow shock gave solar wind speed of $\sim 450 \mathrm{~km} / \mathrm{s}$ and $\sim$ $425 \mathrm{~km} / \mathrm{s}$, respectively (Slavin et al., 2014). Orbital speeds of Mercury were $\sim 53 \mathrm{~km} / \mathrm{s}$ on 23 November 2011 and $\sim 47 \mathrm{~km} / \mathrm{s}$ on 11 May 2012, the aberration angles were calculated to be $\sim 6.76^{\circ}$ and $\sim 6.31^{\circ}$, respectively.

\subsection{CME on 23 November 2011}

The crossing of Mercury's magnetosphere under the CME impact was the first periapsis pass of MESSENGER on 23 November 2011. The blue lines in Figure 1 exhibit MESSENGER's orbit in the $\hat{x}_{M S M^{-}}^{\prime} \hat{y}_{M S M}^{\prime}$ and $\hat{y}_{M S M^{-}}^{\prime} \hat{z}_{M S M}$ planes and the black curves are the magnetopause locations, which is obtained from a magnetopause model (Shue et al., 1998; Winslow et al., 2013) with a subsolar standoff distance $\left(R_{s s}\right)$ of $1.13 R_{M}$ (Slavin et al., 2014). The dashed black curves are the average magnetopause locations (Winslow et al., 2013). The black curves were closer to the planet, indicating that Mercury's magnetosphere was clearly compressed during the extreme events. MESSENGER moved northward through the tail southern magnetopause at $X_{M S M}^{\prime} \sim-3.8 R_{M}$ and 

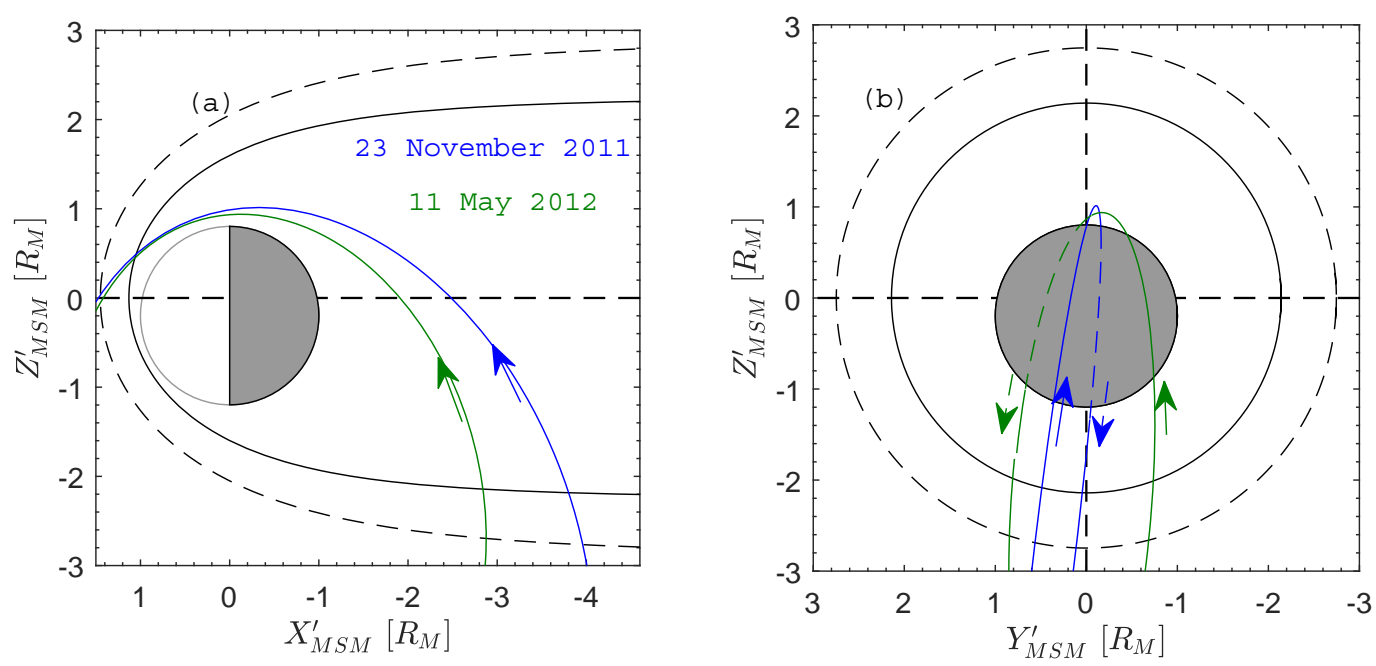

Figure 1. MESSENGER trajectories for the three extreme events (blue lines for a Coronal Mass Ejection (CME) on 23 November 2011 and green lines for a high-speed stream (HSS) on 11 May 2012) on the aberrated $\hat{x}_{M S M}^{\prime}-\hat{y}_{M S M}^{\prime}$ (a) and $\hat{y}_{M S M}^{\prime}-\hat{z}_{M S M}$ (b) planes. The black curve in (a) indicates the magnetopause location with a subsolar standoff $\left(R_{s s}\right)$ distance of $1.13 R_{M}$, which was determined by Slavin et al. (2014) for the three events. The black circular in (b) is the magnetopause location at $X_{M S M}^{\prime} \sim-2.8 R_{M}$. The dashed curve in (a) and the dashed circle in (b) represent the average magnetopause locations $\left(R_{s s}=1.45 R_{M}\right)$ determined by Winslow et al. (2013).

entered the southern lobe of the magnetotail. It then crossed the magnetic equatorial plane at $X_{M S M}^{\prime} \sim-2.5 R_{M}$ and entered the northern hemisphere. MESSENGER reached the periapsis at the northern high-latitude region on the dayside and crossed the cusp and then the dayside magnetopause. The trajectory during the CME (the blue line in Figure 1b) was on the pre-midnight sector in the nightside magnetosphere and was close to the noon-midnight meridian $\left(\left|Y_{M S M}^{\prime}\right|<0.5 R_{M}\right)$.

An overview of ion and magnetic field measurements across the nightside magnetosphere during the CME is displayed in Figure 2 (For more information on the CME, see Slavin et al. (2014) or Winslow et al. (2015)). The encounter of high-latitude tail magnetopause was determined to be at $\sim$ 08:28:00 UTC (the first vertical dashed red line) when rotation in $B_{x}$ was observed. The tail magnetopause was $\sim 2.23 R_{M}$ away from the $\hat{x}_{M S M}^{\prime}$ axis, which was close to the distance of $\sim 2.19 R_{M}$ in the magnetopause model (Figure $1 \mathrm{~b}$ ). In the following calculations, the radius $\sim 2.23 R_{M}$ determined from insitu measurements was used as the radius of the magnetotail for this event. Therefore, the width of the magnetotail $\left(d_{\text {Tail }}\right)$ was $4.46 R_{M}$. In the magnetosheath from 08:05:00 UTC to 08:11:00 UTC, the average IMF was $[-19.4,70.9,-36.7] n T$, which was predominately in duskward and southward directions with a magnetic shear angle of $\sim 117^{\circ}$. The flux transfer events (FTEs) were frequently observed around the magnetopause, which were identified based on their bipolar signatures coincident with enhancements in the magnetic field intensity.

The southern lobe was identified to be the region between the first $(\sim$ 08:28:00 UTC) and second $(\sim 09: 22: 10 \mathrm{UTC})$ vertical dashed lines. The solar-wind-originated proton (Figures $2 \mathrm{a}$ and $2 \mathrm{~b}$ ) and $\mathrm{He}^{++}$(Figure 2c) continuously appeared after crossing the tail magnetopause, and the proton flux (Figure 2a) and observed density of $\mathrm{He}^{++}$(Figure 2c) (Raines et al., 2013) smoothly decreased farther away from the magnetopause, which 


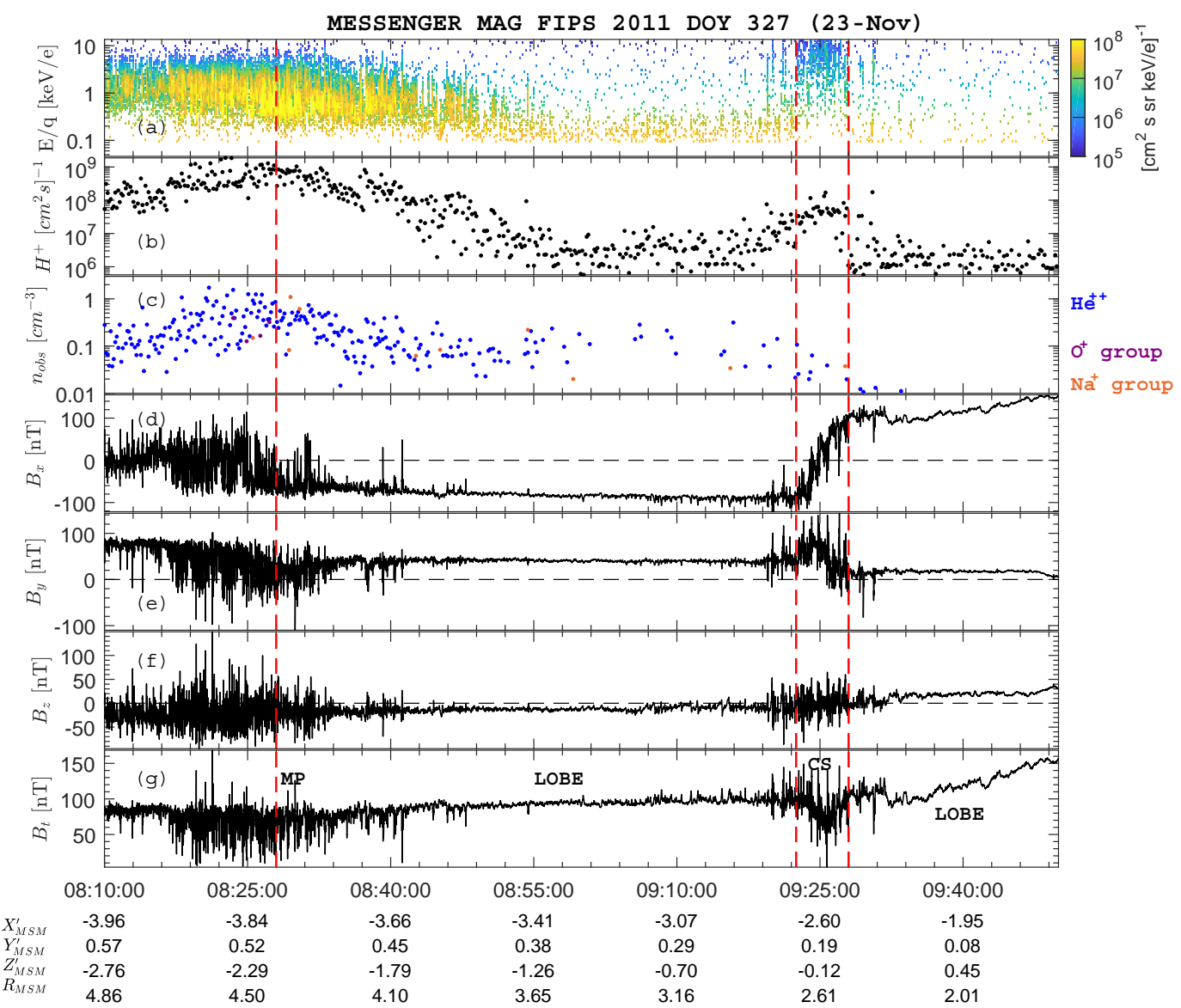

Figure 2. Overview of ion and magnetic field measurements across the nightside Mercury's magnetosphere from 08:10:00 UTC to 09:50:00 UTC on 23 November 2011. (a) Proton differential particle flux versus energy per charge. (b) Proton particle flux integrated in the energy range of FIPS ( $\sim 46 \mathrm{eV}$ to $\sim 13.3 \mathrm{keV})$. (c) Observed density of $\mathrm{He}^{++}$(in blue), $\mathrm{O}^{+}$group $(\mathrm{m} / q=14$ to 20 , in purple), and $N a^{+}$group $\left(m / q=21\right.$ to 30 , in gold). (d) $B_{x}$. (e) $B_{y}$. (f) $B_{z}$. (g) magnetic field intensity $\left(B_{t}\right)$. The first vertical dashed red line indicates the average magnetopause location. The second and third vertical dashed lines indicate the south and north boundaries of the plasma sheet, respectively. Magnetopause (MP), Lobe and Current sheet (CS) are labeled. 


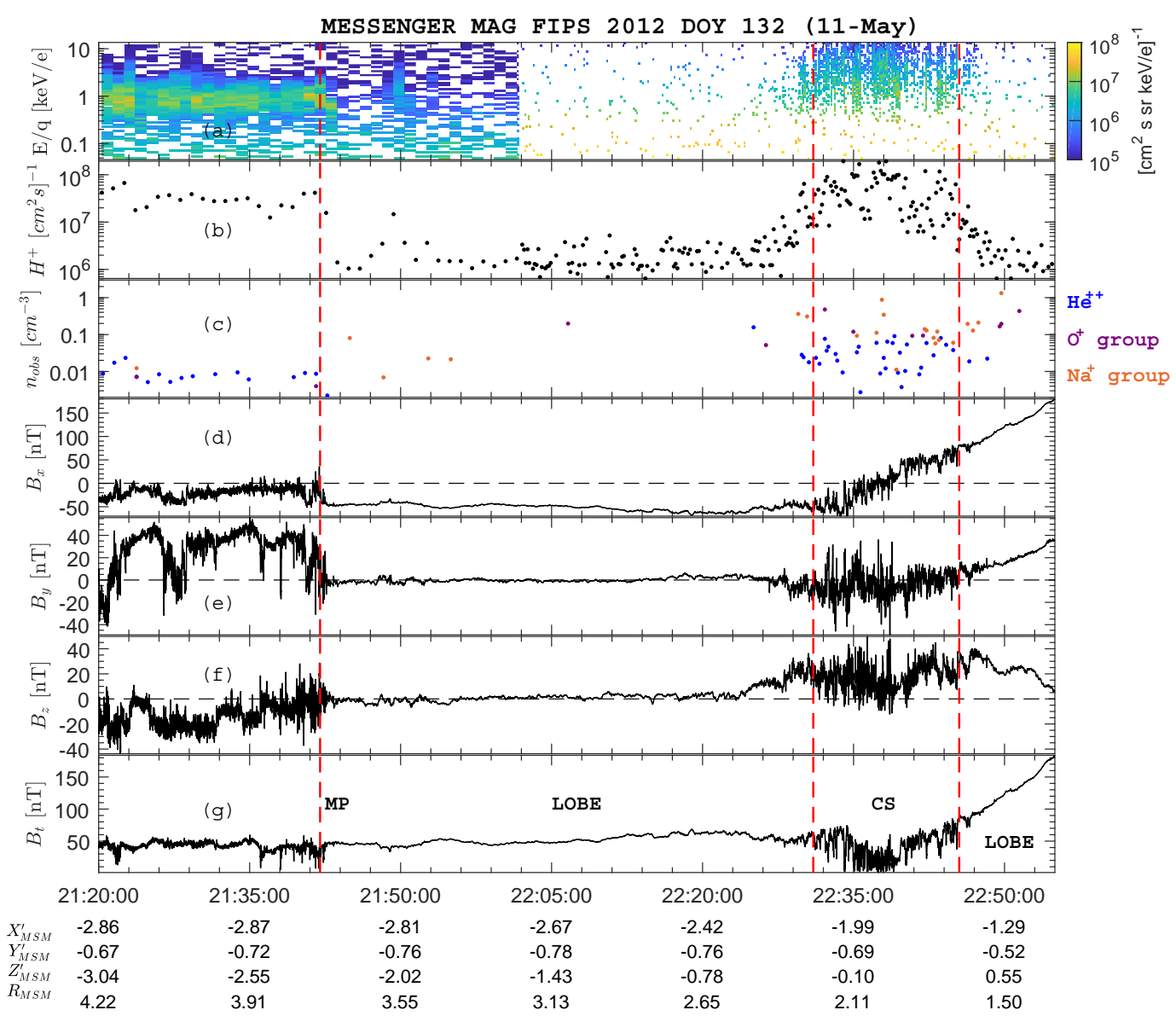

Figure 3. Overview of ion and magnetic field measurements across the nightside Mercury's magnetosphere from 21:20:00 UTC to 22:55:00 UTC on 11 May 2012. This figure is in the same format as Figure 2. FIPS was operating at a $60 \mathrm{~s}$ cadence through the first half of this period, up to about 22:02 UTC. It operated at $\sim 10$ s for the remainder of the interval.

indicates an encounter of plasma mantle. In the southern lobe, the magnetic field was steady with the field orientation primarily in the tailward and duskward directions (Figures $2 \mathrm{~d}$ to $2 \mathrm{~g}$ ). There were no signatures of magnetic flux loading and unloading. The plasma sheet was identified between the second and third vertical dashed lines as a depression in the magnetic field intensity (Figure 2g), an increase in the proton flux (Figures $2 \mathrm{a}$ and $2 \mathrm{~b}$ ), and reversal of the $B_{x}$ (Figure $2 \mathrm{~d}$ ). The plasma sheet contained an amount of supra-thermal protons $\left(>3 \times 10^{7}\left[\mathrm{~cm}^{2} \mathrm{~s}\right]^{-1}\right)$ (Sun et al., 2017). Frequent and largeamplitude increases in the magnetic field intensity were also observed in the plasma sheet.

\subsection{HSS on 11 May 2012}

Figure 3 shows the ion and magnetic field measurements on 11 May 2012, which was the third periapsis pass of MESSENGER on that day. Green lines in Figure 1 represent the trajectory of MESSENGER, which deviated from the meridian plane but within $Y_{M S M}^{\prime}<-0.8 R_{M}$. The high-latitude tail magnetopause was crossed at $\sim 21: 42: 00$ UTC (the first vertical dashed line), and was $\sim 2.43 R_{M}$ away from the $\hat{x}_{M S M}^{\prime}$ axis. FTEs were frequently observed around the magnetopause, and the average IMF was $[-12.5,37.3,-12.1] n T$ from 21:29:00 UTC to 21:39:00 UTC, which was southward but contained a large duskward 
component with a magnetic shear angle of $\sim 108^{\circ}$. In the southern lobe (the region between the first and second vertical dashed lines), magnetic field intensity displayed some amplitude variations, but did not show continuous magnetosheath proton and solar wind $\mathrm{He}^{++}$. There was a brief magnetosheath proton enhancement at $\sim$ 21:50:00 UTC, which might be the encounter of the plasma mantle. The plasma sheet (between the second and third vertical dashed lines) was full of magnetic field fluctuations and contained large amounts of high-energy proton particle flux. There were planetary $\mathrm{O}^{+}$and $\mathrm{Na}^{+}$evident in the plasma sheet (Figure 3c). In contrast, they were not present in the plasma sheet during the CME on 23 November 2011.

In the extreme solar wind events, the nightside magnetosphere was highly compressed. On the CME on 23 November 2011, magnetic shear angle outside the tail magnetopause was determined to be $\sim 117^{\circ}$, and on the HSS on 11 May 2012, the shear angle was $\sim$ $108^{\circ}$. The shear angles are similar to each other. However, magnetic shear angle at the dayside magnetopause was $\sim 60^{\circ}$ on the CME event and was $\sim 160^{\circ}$ on the HSS event. In addition, the dayside magentosheath $\beta$ was determined to be $\sim 0.06$ on the CME and was $\sim 2.67$ on the HSS (Slavin et al., 2014). Protons in the plasma sheet were energized, and the plasma sheet contained frequent and large-amplitude magnetic field fluctuations. In the next section, we analyze the reconnection-generated magnetic structures in the plasma sheet, including flux ropes (the CME on 23 November 2011) and dipolarization fronts (the HSS on 11 May 2012). The quadrupole Hall magnetic field associated with magnetic reconnection and the dimensionless reconnection rate on the CME event (23 November 2011) are also analyzed. Section 4 shows analyses on the southern lobes. The open magnetic flux in the southern lobe does not show magnetic flux loading and unloading, suggesting that the magnetosphere experienced the quasi-steady convection. The cross-polar Cap Potential (CPCP) was calculated from the plasma mantle measurements on the CME event, which is a few times the average value in Mercury's magnetosphere. In section 5, we discuss features of steady convection in Mercury's magnetosphere and compare them with the SMCs in Earth's magnetospheres, including the solar wind drivers and the steady convection properties. The dawn-dusk extent of the magnetic reconnection during the CME event is also discussed. Section 6 gives the conclusion.

\section{Plasma Sheet Observations}

\subsection{Local Coordinate System for the Cross-tail Current Sheet}

The local coordinate system (LMN) is crucial in analyzing magnetic structures in the cross-tail current sheet. In the case of the magnetotail magnetic reconnection, $\vec{L}$ is along the reconnecting component of the magnetic field, $\vec{N}$ is normal to the current sheet and $\vec{M}$ is directed along the reconnection X-line. Several techniques have been developed to determine the LMN coordinate of magnetic structures. Here we apply two of these techniques to the MESSENGER magnetic field measurements. The first is the minimum, or maximum, variance analysis (MVA) (Sonnerup \& Cahill, 1967; Sonnerup \& Scheible, 1998). The second is the cross product of the magnetic field vectors on the two sides of magnetic layers (Sonnerup \& Scheible, 1998). The MVA was applied to a series of measurements containing the structure. It gives three eigenvalues (the maximum $\lambda_{\max }$, intermediate $\lambda_{i n t}$ and minimum $\lambda_{\text {min }}$ eigenvalues), which correspond to three eigenvectors (the maximum, intermediate and minimum eigenvectors). The maximum, intermediate and minimum eigenvectors correspond to the $\overrightarrow{L_{M V A}} \overrightarrow{\text {, the }} M_{M V A}$, and the $N_{M V A}$, respectively. Ratios between the neighboring eigenvalues imply the accuracy of the eigenvectors, in which a small value would degenerate the corresponding eigenvectors. In this study, we require the ratios to be greater than 3 .

In the second technique, the LMN is obtained by analyzing magnetic field vectors on the two sides of the cross-tail current sheet, that is, the southern and northern lobes. The direction of magnetic reconnection line (Sonnerup, 1974), that is, the $\vec{M}$ direction, 
could be calculated from

$$
\overrightarrow{M_{\text {vectors }}}=\left(\overrightarrow{B_{S L}} \times \overrightarrow{B_{N L}}\right) \times\left(\overrightarrow{B_{S L}}-\overrightarrow{B_{N L}}\right) /\left|\left(\overrightarrow{B_{S L}} \times \overrightarrow{B_{N L}}\right) \times\left(\overrightarrow{B_{S L}}-\overrightarrow{B_{N L}}\right)\right|
$$

, where $\overrightarrow{B_{S L}}$ and $\overrightarrow{B_{N L}}$ represent magnetic field vectors in southern and northern lobes, respectively. Since magnetic field in the lobes are expected to be predominately in a plane parallel to the cross-tail current sheet, the normal of the current sheet, that is, the $\vec{N}$, could be obtained from

$$
N_{\text {vectors }}=\left(\overrightarrow{B_{S L}} \times \overrightarrow{B_{N L}}\right) /\left|\left(\overrightarrow{B_{S L}} \times \overrightarrow{B_{N L}}\right)\right|
$$

, and then the reconnecting direction, that is, the $\vec{L}$, is

$$
\overrightarrow{L_{\text {vectors }}}=\left(\overrightarrow{B_{S L}}-\overrightarrow{B_{N L}}\right) /\left|\left(\overrightarrow{B_{S L}}-\overrightarrow{B_{N L}}\right)\right|
$$

, which is $\overrightarrow{M_{\text {vectors }}} \times \overrightarrow{N_{\text {vectors }} \text {. }}$

Magnetic field measurements from 09:19:00 UTC to 09:34:00 UTC on 23 November 2011 and from 22:26:00 to 22:47:00 UTC on 11 May 2012 were taken out to apply the MVA separately to obtain the LMN coordinate for the cross-tail current sheets. On 23 November 2011, the ratios between the maximum eigenvalue and the intermediate eigenvalue was $\sim 23.8$, and the intermediate eigenvalue and the minimum eigenvalue was $\sim 3.4$, indicating that the $\overrightarrow{L_{M V A}}$, the $M_{M V A}^{\overrightarrow{ }}$, and the $N_{M V A}$ were well determined. In the MSM coordinate system, $\vec{L}_{M V A}=(0.98,-0.20,0.07), M_{M V A}=(0.19,0.98,0.10)$, and $\vec{N}_{M V A}=(-0.08,-0.08,0.99)$, which were close to $\hat{x}_{M S M}, \hat{y}_{M S M}$ and $\hat{z}_{M S M}$ axes, respectively. On 11 May 2012, the ratios between the maximum eigenvalue and the intermediate eigenvalue was $\sim 32.0$, and the intermediate eigenvalue and the minimum eigenvalue was $\sim 1.1$, indicating that the $L_{M V A}$ was well determined, but the $M_{M V A} \vec{A}$ and the $N_{M V A}$ were becoming degenerate. The $L_{M V A}=(0.994,0.08,0.07)$ was close to the $\hat{x}_{M S M}$.

In the CME event on 23 November 2011, magnetic field vectors in the southern and northern lobes were $\overrightarrow{B_{S L}}=(-87.38,37.67,-9.92) \mathrm{nT}$ and $\overrightarrow{B_{N L}}=(102.08,18.49,15.98)$ $\mathrm{nT}$, which were averaged between 09:16:00 UTC and 09:19:00 UTC and between 09:29:00 UTC and 09:32:00 UTC, respectively. The magnetic shear angle was $\sim 147^{\circ}$ between the $\overrightarrow{B_{S L}}$ and the $\overrightarrow{B_{N L}}$, indicating a guide field in the plasma sheet. Applications of equations (1), (2) and (3) gave $L_{\text {vectors }}=(0.98,-0.10,0.14), M_{\text {vectors }}=(0.09,0.99,0.08)$, and $N_{\text {vectors }}=(-0.14,-0.07,0.99)$, which were $5.87^{\circ}, 5.56^{\circ}, 4.51^{\circ}$, respectively, away from the LMN determined by the MVA. This implies a very good agreement.

In the HSS event on 11 May 2012, the $\overrightarrow{B_{S L}}=(-60.57,1.87,2.09) \mathrm{nT}$ and the $\overrightarrow{B_{N L}}$ $=(82.34,10.07,30.13) \mathrm{nT}$, which were averaged between 22:20:30 UTC and 22:24:00 UTC, and between 22:45:20 UTC and 22:46:10 UTC, respectively. MESSENGER was located at $\sim(-1.51,-0.58,0.39) R_{M}$ in the northern lobe close to the planet, it was necessary to remove the dipole magnetic field components in the $\overrightarrow{B_{N L}}$, which resulted in $\overrightarrow{B_{N L}^{\prime}}=$ $(60.69,1.88,-3.40) \mathrm{nT}$. Application of equations (1), (2) and (3) gave $\overrightarrow{L_{\text {vectors }}}=(0.999$, $0.0,0.045), M_{\text {vectors }}=(-0.015,0.94,-0.33)$, and $N_{\text {vectors }}=(0.04,0.33,0.94)$. The $L_{\text {vectors }}$ was separated from the $\overrightarrow{L_{M V A}}$ by $\sim 8.18^{\circ}$, which, again, is a very good agreement. The local coordinates obtained from MVA and the cross product of the north and south lobes for both events are summarized in Table 1. Since the $M_{M V A}$ and $N_{M V A}$ determined by MVA were degenerate in the HSS event, the LMN coordinates determined from the cross product analysis of the lobe fields were employed for both CME and HSS events in the following analysis on the plasma sheet.

\subsection{Quasi-periodic Flux Rope Groups during 23 November 2011 CME}

An overview of the proton and magnetic field measurements in the plasma sheet on 23 November 2011 is displayed in Figure 4. The plasma sheet is evident in the enhanced proton flux from $\sim 1$ to $10 \mathrm{keV}$ (Figure 4a) and a depression of magnetic field 


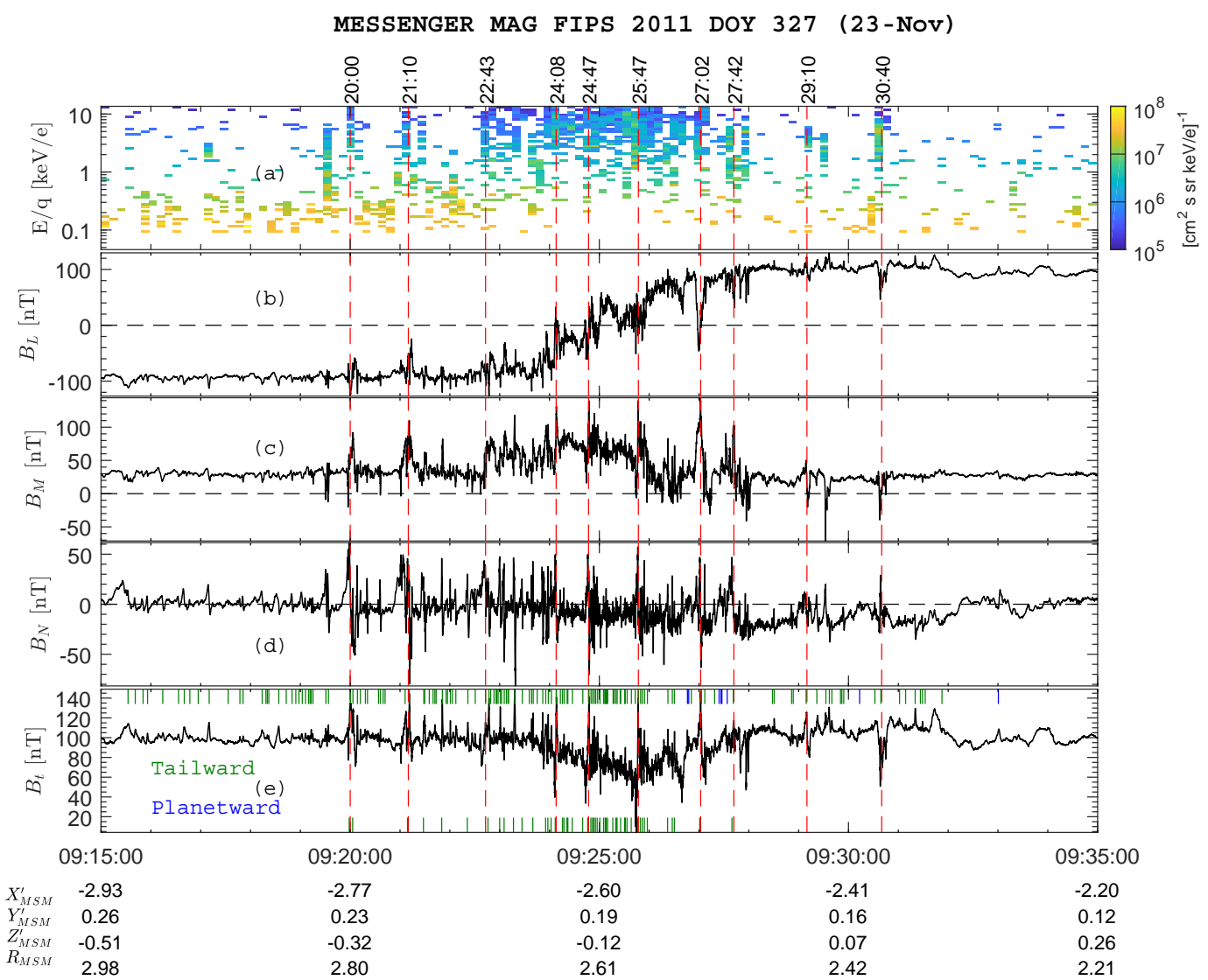

Figure 4. Overview of proton and magnetic field measurements of the plasma sheet from 09:15:00 UTC to 09:35:00 UTC on 23 November 2011. (a) Proton differential particle flux versus energy per charge $(E / q)$. (b) $B_{L}$. (c) $B_{M}$. (d) $B_{N}$. (e) magnetic field intensity $B_{t}$. LMN represents the local coordinate of the cross-tail current sheet, in which $\vec{L}$ is the magnetic field maximum variance direction, $\vec{M}$ is the intermediate variance direction, and $\vec{N}$ is the minimum variance direction. Red dashed lines indicate the starts of flux rope groups. The green and blue ticks at the top of (e) represent the flux ropes and traveling compression regions (TCRs) with green being tailward traveling and blue being planetward traveling. The flux ropes among them are marked by additional green ticks at the bottom. 

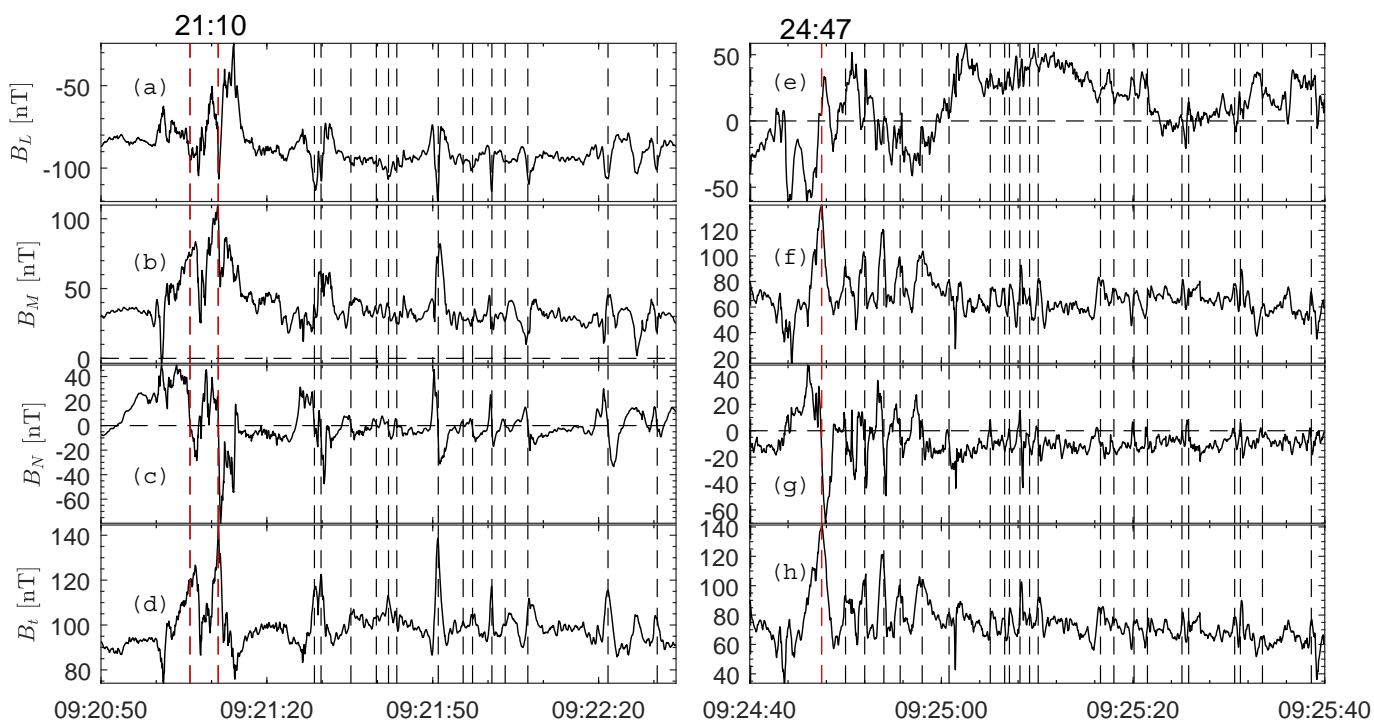

Figure 5. Overview of two flux rope groups in the LMN local coordinate system. The LMN is the same as Figure 4. The left column is the flux rope group of 09:21:10, and the right column is the group of 09:24:47. (a) and (e) $B_{L}$. (b) and (f) $B_{M}$. (c) and (g) $B_{N}$. (d) and (h) $B_{t}$. The vertical dashed lines represent the flux ropes and TCRs with the leading larger-scale flux ropes being marked in red.

intensity (Figure 4e). MESSENGER crossed the center of the plasma sheet at $\sim$ 09:24:45 UTC where $B_{L}$ reversed direction (Figure $4 \mathrm{~b}$ ). The plasma sheet contained many suprathermal protons with energies higher than $\sim 5 \mathrm{keV}$ (Figure 4a) (Sun et al., 2017), indicating that the plasma sheet protons were energized. The FIPS angular flux maps (see supplementary material), including energy scans from 09:24:40 UTC to 09:27:40 UTC, showed that most of the protons were tailward propagating. This indicates that MESSENGER traversed the cross-tail current sheet tailward of the Near-Mercury Neutral Line (NMNL). An integration over the plasma sheet crossing yielded a proton number density $\left(n_{p}\right)$ of $\sim 2.33 \mathrm{~cm}^{-3}$ and proton temperature $\left(T_{p}\right)$ of $\sim 32.5 \mathrm{MK}$ (see supplementary material). Meanwhile, the plasma sheet was full of large-amplitude magnetic field fluctuations (Figures $4 \mathrm{~b}$ to $4 \mathrm{e}$ ), which we will now show to be flux ropes.

At 09:21:10 UTC, when MESSENGER located near the southern boundary of the plasma sheet, two large-amplitude, long-duration tailward traveling flux ropes were observed (marked in red vertical lines), which is shown on the left column of Figure 5. The deflection in the $B_{N}$ were used to determine the travelling directions of the flux ropes and travelling compression regions (TCRs). Planetward travelling structures correspond to $B_{N}$ changes from negative to positive $\left(\mp B_{N}\right)$, and tailward travelling structures correspond to $B_{N}$ changes from positive to negative $\left( \pm B_{N}\right)$. The TCRs are the locally compressed fields draping around the flux ropes (Slavin, Imber, et al., 2012; Slavin et al., 1993). In the two flux ropes, $B_{N}$ changed from $\sim 50 \mathrm{nT}$ to $-20 \mathrm{nT}\left(\Delta B_{N} \sim 70 \mathrm{nT}\right)$ and $\sim 40$ $\mathrm{nT}$ to $-70 \mathrm{nT}\left(\Delta B_{N} \sim 110 n T\right)$ in $\sim 3.5$ seconds $(\Delta t)$ and $\sim 1.5$ seconds and the maximum magnetic field intensity $\left(B_{t}\right)$ was $\sim 125 n T$ and $145 \mathrm{nT}$, respectively. The flux ropes were followed by a prolonged negative $B_{N}$, that is, the southward $B_{z}$, with an interval of $\sim 75 \mathrm{~s}$. This prolonged negative $B_{N}$ could mean that magnetic reconnection remained occur, which is in analogous to the post-plasmoid plasma sheet (PPPS) (DiBraccio, Slavin, Imber, et al., 2015; Richardson et al., 1987). Fourteen relatively smaller-amplitude $\left(\Delta B_{N}<70 n T\right)$ and shorter-duration $(\Delta t \sim 1 s)$ tailward flux ropes and TCRs were 
observed in the prolonged negative $B_{N}$ until another large-amplitude and long-duration flux rope appeared at $\sim$ 09:22:43 UTC. The right column in Figure 5 displays another flux rope group from 09:24:40 UTC to 09:25:40 UTC, in which few large-amplitude, longduration flux ropes were observed at the start time and were followed by prolonged negative $B_{N}$ and several relatively smaller-amplitude and shorter-duration flux ropes. Twentythree flux ropes and TCRs were observed. The two flux rope groups displayed similar features, which was led by a few large-amplitude and long-duration flux ropes followed by a prolonged negative $B_{N}$ and tens of relatively smaller-amplitude and shorter-duration flux ropes. This kind of flux rope group quasi-periodically appeared between 09:20:00 UTC and 09:30:40 UTC and the vertical dashed red lines in Figure 4 represent the start times of each group. There were ten this kind of flux rope groups with a mean duration of $\sim 71 \mathrm{~s}$

If we considered the average Alfvén speed in the plasma sheet to be the plasma flow speed, the scale of the flux ropes could be estimated. The average $n_{p}$ is $2.33 \mathrm{~cm}^{-3}$ and the average $B_{t}$ is $\sim 85 n T$ from 09:24:00 to 09:26:00 UTC, the Alfvén speed is calculated to be $\sim 800 \mathrm{~km} / \mathrm{s}$. The large scale flux rope of $3.5 \mathrm{~s}$ corresponds to a radius of $\sim 2800$ $\mathrm{km}$. The small scale flux rope of $1 \mathrm{~s}$ suggests that the radius of flux rope is around 400 $\mathrm{km}$. Proton inertial length is $\sim 150 \mathrm{~km}$. The large scale flux rope is tens of the proton inertial length, and the small scale flux rope is only several times the inertial length, which suggests that the small scale flux ropes are ion-scale..

Green and blue ticks in Figure 4e marked the flux ropes and TCRs identified between 09:15:00 UTC and 09:35:00 UTC, which were 153 in a total number. These flux ropes and TCRs were visually identified through applying MVA on each event. In Figure 4, the green ticks represented tailward traveling flux ropes and TCRs, and the blue ticks represented planetward traveling flux ropes and TCRs. Most of the flux ropes and TCRs were tailward traveling with only $8(\sim 5 \%)$ being planetward traveling. This indicates that the spacecraft stayed mostly in the tailward of the NMNL, which was consistent with proton distributions from FIPS. However, because the NMNL was located between a pair of neighboring tailward and planetward flux ropes, the planetward traveling flux ropes suggested that the spacecraft should cross the NMNL a few times. The first planetward flux rope was detected at $\sim$ 09:26:50 UTC where MESSENGER was located at $X_{M S M}^{\prime} \sim-2.53 R_{M}$. The last planetward TCR was detected at $\sim$ 09:33:00 UTC when MESSENGER was located at $X_{M S M}^{\prime} \sim-2.28 R_{M}$. There were $\sim 20$ flux ropes both planetward traveling and tailward traveling in between. This indicates a movement of the NMNL, which should be located at $X_{M S M}^{\prime}$ from $-2.53 R_{M}$ to $-2.28 R_{M}$.

Figure 6 shows the statistical properties of the flux ropes (red dots, a and b) and the TCRs (black dots, $\mathrm{c}$ and $\mathrm{d}$ ). Amplitude $\left(\Delta B_{N}\right)$ and duration $(\Delta t)$ of the structures was determined from the extrema in $B_{N}$ variations. Core field $\left(B_{\text {core }}\right)$ was the maxima of $B_{t}$ in the structures. The flux ropes had mean $\Delta B_{N}$ of $\sim 52.4 n T$, mean $B_{\text {core }}$ of $\sim$ 107.0 $n T$, and mean $\Delta t$ of $\sim 0.93 \mathrm{~s}$. The TCRs had mean $\Delta B_{N}$ of $\sim 19.7 n T$, mean magnetic field enhancement $\left(\Delta B_{t} / B_{\text {Lobe }}\right)$ of $\sim 6 \%$, and mean $\Delta t$ of $\sim 1.62 \mathrm{~s}$. The relative amplitude of the TCRs, $\sim 6 \%$, is comparable to that seen at Earth (Slavin et al., 1993). The mean duration of the TCRs was longer than that of the flux ropes. TCRs have been well studied at Earth and it has been shown that they are due to the draping of lobe magnetic field lines around flux ropes (Slavin, Imber, et al., 2012; Slavin et al., 1993). Hence, they are useful proxies for flux ropes.

The $\Delta t$ represent scales of flux ropes along $L$ direction. The $\Delta B_{N}$ are the amplitudes of the flux ropes, which represents the curvature radius of the magnetic field lines and therefore the scale of flux ropes along $N$ direction (Zhao et al., 2019). Figure 6a shows a good positive correlation between $\Delta t$ and $\Delta B_{N}$ for the flux ropes, which indicates that the longer of the flux ropes along $L$ the larger of the flux ropes in $N$. In Figure $6 \mathrm{~b}$, the $\Delta t$ and the $B_{\text {core }}$ are also positively correlated, which indicates that the larger the scale of flux ropes the stronger the core field. The $\Delta B_{N}$ and $B_{\text {core }}$ are also positively corre- 

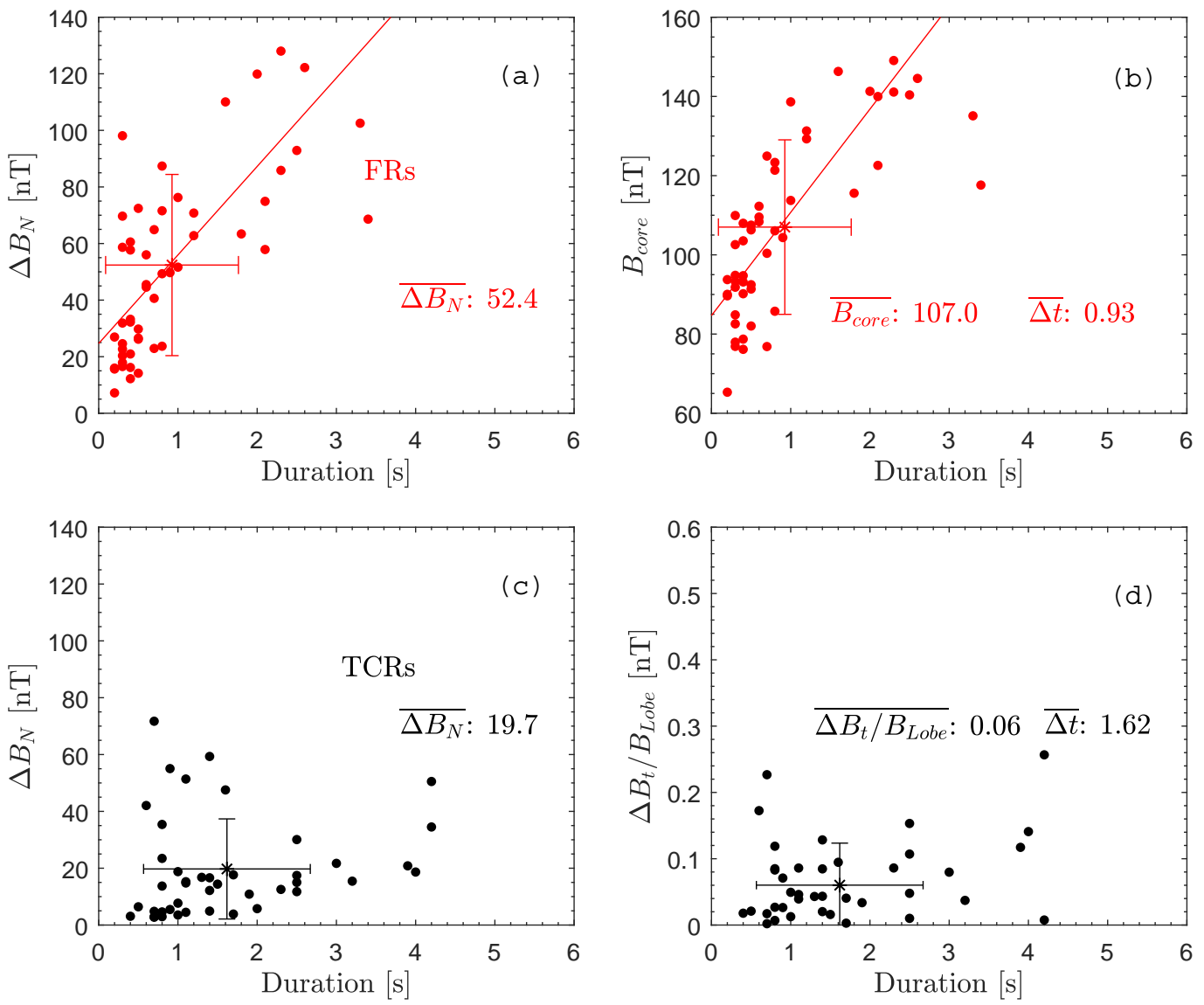

Figure 6. Scatter of the flux ropes (FRs, red dots, a and b) and traveling compression regions (TCRs, black dots, c and d) in the plasma sheet on 23 November 2011. (a) The amplitude $\left(\Delta B_{N}\right)$ versus flux rope duration $(\Delta t)$. (b) The core field ( $\left.B_{\text {core }}\right)$ versus $\Delta t$. (c) The amplitude $\left(\Delta B_{N}\right)$ versus TCR duration $(\Delta t)$. (d) The $\Delta B_{t} / B_{\text {Lobe }}$ versus TCR duration. For each flux rope or TCR, the $\Delta B_{N}$ and $\Delta t$ of the structures was determined from the extrema in the $B_{N}$ variations, and the $B_{\text {core }}$ was the maxima in $B_{t}$. Red lines are linear fitting of the flux ropes (red dots). 
lated (not shown). Several studies propose that core magnetic field strengthens along with growth of flux rope scales (Ma et al., 1994; Y. Chen et al., 2017; Akhavan-Tafti et al., 2018; Sun et al., 2019). The distribution in Figure 6b agrees with this scenario. Further, the positive correlations among the $\Delta B_{N}$, the $\Delta t$, and $B_{\text {core }}$ might indicate that the larger flux ropes would contain more magnetic flux.

In the plasma sheet from 09:22:40 UTC to 09:28:00 UTC, a number of 74 flux ropes were identified corresponding to mean separation of $\sim 4.3 \mathrm{~s}$. The occurrence rate $(\sim 14$ events per minute) of flux ropes during this CME was approximately 600 times the average occurrence rate $(\sim 0.022$ events per minute (Sun et al., 2016)) in the Mercury's plasma sheet. In Earth's plasma sheet, multiple flux ropes and TCRs were also observed, such as in Slavin et al. (1993, 2005); Zong et al. (2004). From those studies, separations between the neighboring flux ropes at Earth could be $\sim 1$ to 2.5 minutes, which is much longer than the $\sim 4.3 \mathrm{~s}$ in Mercury's plasma sheet.

\subsection{Dipolarization fronts during 11 May 2012 HSS}

Figure 7 shows proton dynamic spectra and magnetic field measurements in the plasma sheet on 11 May 2012. Similar to the plasma sheet on 23 November 2011, the plasma sheet was evident in the suprathermal proton enhancement (Figure 7a) and magnetic field intensity depression (Figure 7e). The integration over the plasma sheet period from 22:29:40 UTC to 22:45:20 UTC of FIPS measurements gave proton number density of $\sim 0.90 \mathrm{~cm}^{-3}$ and proton temperature of $20.9 \mathrm{MK}$ (see supplementary material for the 3D FIPS angular flux map and 1D phase space density). Different from the plasma sheet on 23 November 2011, this plasma sheet contained many heavy ions, including solar wind $\mathrm{He}^{++}$and planetary $\mathrm{Na}^{+}$. The integration gives $\mathrm{He}^{++}$density of $\sim 0.13 \mathrm{~cm}^{-3}$ and temperature of $47.4 \mathrm{MK}$ and $\mathrm{Na}^{+}$density of $\sim 0.12 \mathrm{~cm}^{-3}$ and temperature of $30.7 \mathrm{MK}$. The density of $\mathrm{Na}^{+}$in this plasma sheet was around an order of magnitude larger than the average $\mathrm{Na}^{+}$density of $\sim 0.01 \mathrm{~cm}^{-3}$ on the dawnside plasma sheet (Raines et al., 2013), while the density of $\mathrm{He}^{++}$was comparable to the average density on the dawnside plasma sheet.

Several TCRs were observed when MESSENGER was located in the southern lobe as marked in Figure 7f. At first, the TCRs were tailward traveling and then became planetward traveling, indicating the pass of the NMNL. As already mentioned, the NMNL should be located between the neighboring planetward and tailward traveling TCRs and flux ropes. The first neighboring planetward and tailward TCRs was located at $X_{M S M}^{\prime} \sim$ - $2.45 R_{M}$, and the last neighboring TCRs was $X_{M S M}^{\prime} \sim-2.34 R_{M}$. Therefore, the NMNL should be located between $X_{M S M}^{\prime} \sim-2.45 R_{M}$ and $\sim-2.34 R_{M}$. Several minutes later, the spacecraft started to enter the plasma sheet. Because MESSENGER moved closer to the planet, it crossed the planetward side of the NMNL reconnection site. MESSENGER crossed the center of the plasma sheet at $X_{M S M}^{\prime} \sim-1.8 R_{M}$ on the HSS event, which was closer to Mercury than 23 November 2011 CME plasma sheet traversal ( $X_{M S M}^{\prime} \sim$ $\left.-2.6 R_{M}\right)$.

Figures 8a to 8d show magnetic field measurements in the central plasma sheet from 22:35:00 UTC to 22:36:30 UTC. The vertical dashed lines marked the planetward traveling dipolarization fronts. Dipolarization fronts consists of sharp increase in $B_{N}$, that is, the northward magnetic field component $\left(B_{z}\right)$, and $B_{t}$, which are preceded by decrease in $B_{N}$ and are followed by $B_{t}$ enhancements (e.g., Ohtani et al., 2004). The $B_{t}$ enhanced region is called plasma bubble or dipolarizing flux bundle (DFB) (e.g., C. X. Chen \& Wolf, 1999; J. Liu et al., 2013), which is believed to be generated by magnetic reconnection (e.g., Angelopoulos et al., 2013). In Figure 8, a number of 13 dipolarization fronts were identified in $90 \mathrm{~s}$. The mean separation of individual dipolarization front was $\sim 7 s$, which was twice the separation of $\sim 4.3 s$ of tailward flux ropes during the CME event. The occurrence rate $(\sim 8.6$ events per minute) of dipolarization front during this HSS was 


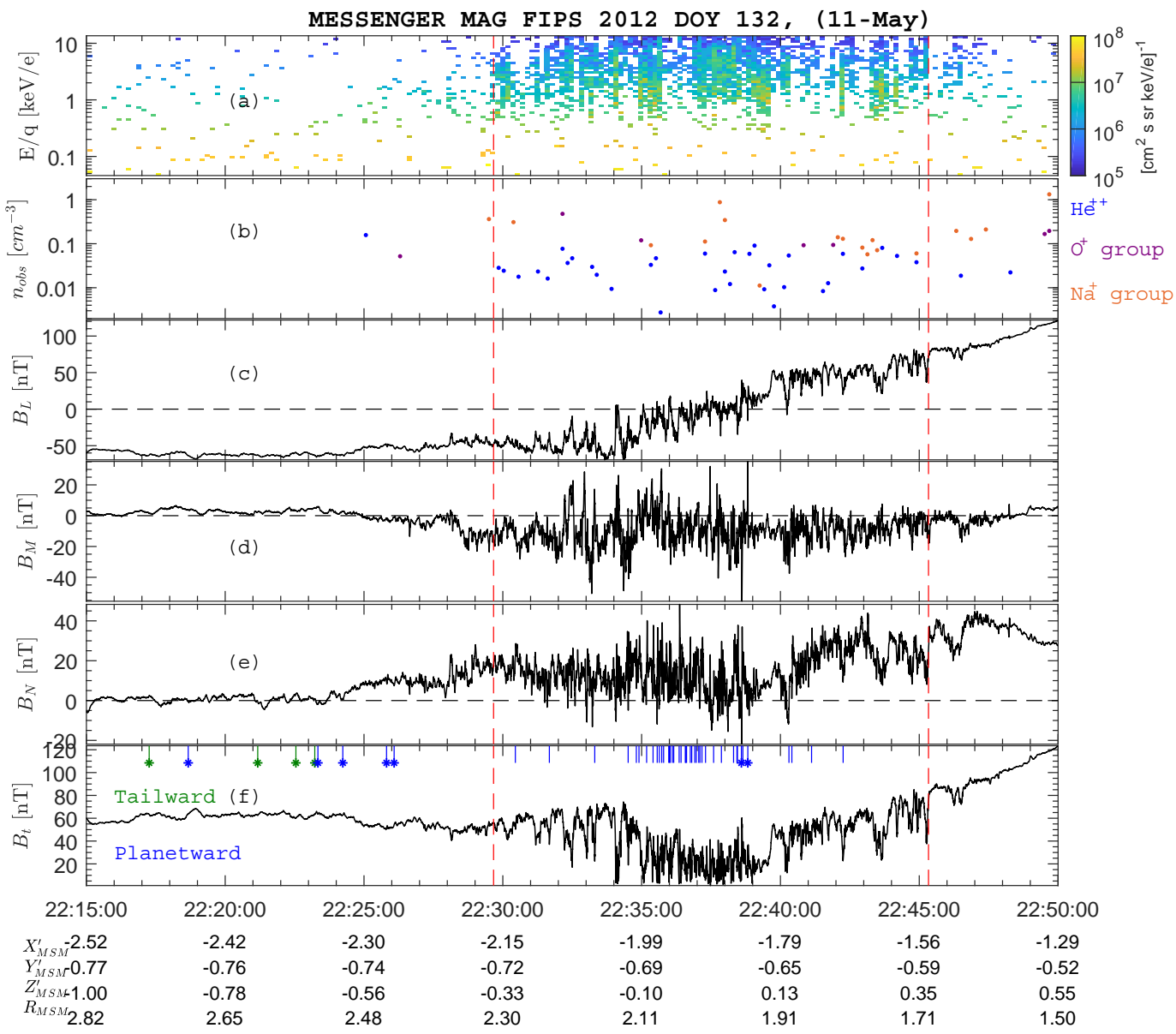

Figure 7. Overview of proton and magnetic field measurements of the plasma sheet from 22:20:00 UTC to 22:50:00 UTC on 11 May 2012. (a) Proton differential particle flux versus energy per charge $(E / q)$. (b) Observed density of $\mathrm{He}^{++}$(in blue), $\mathrm{O}^{+}$group (m/q $=14$ to 20, in purple), and $N a^{+}$group (m/q $=21$ to 30, in gold). (c) $B_{L}$. (d) $B_{M}$. (e) $B_{N}$. (f) magnetic field intensity $B_{t}$. LMN represents the local coordinate of the cross-tail current sheet. Red dashed lines represent the plasma sheet boundaries. The blue ticks in (f) represent the planetward traveling dipolarization fronts. The blue ticks ending with asterisks represent planetward traveling flux ropes and TCRs. The green ticks ending with asterisks represent tailward traveling TCRs. 

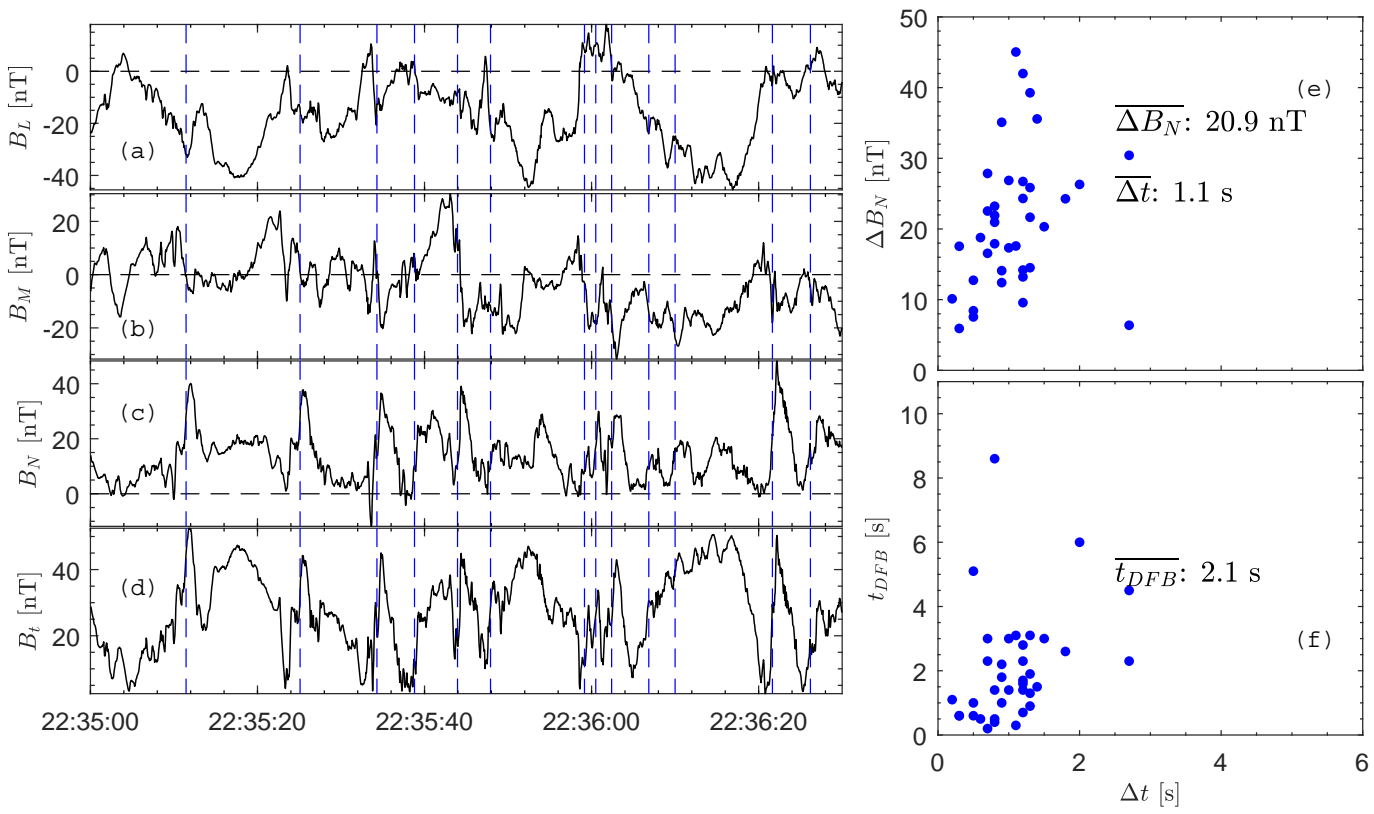

Figure 8. Dipolarization fronts observed in the plasma sheet on 11 May 2012. (a) $B_{L}$. (b) $B_{M}$. (c) $B_{N}$. (d) $B_{t}$. LMN is the local coordinate system, which is the same as Figure 7 . Blue vertical dashed lines mark the centers of dipolarization fronts. (e) dipolarization front duration $(\Delta t)$ versus amplitudes $\left(\Delta B_{N}\right)$. (f) dipolarization front duration versus dipolarizing flux bundles (DFBs) duration.

$\sim 200$ times the average occurrence rate $(\sim 0.044$ events per minute $)$ in Mercury's plasma sheet (Sun et al., 2016).

In Figure $7 \mathrm{f}$ between the two vertical dashed red lines, blue ticks marked the dipolarization fronts and blue ticks ending with asterisks marked the flux ropes. In the central plasma sheet between 22:34:00 UTC and 22:39:00 UTC, 37 dipolarization fronts and two flux ropes were identified. These magnetic structures were planetward traveling, confirming that the spacecraft crossed the planetward plasma sheet of the NMNL. Statistical properties of the dipolarization fronts, including duration $\Delta t$ and amplitude $\Delta B_{N}$ and duration of the DFB $\left(t_{D F B}\right)$, were shown in Figures $8 \mathrm{e}$ and $8 \mathrm{f}$. The $\Delta t$ and $\Delta B_{N}$ of the dipolarization fronts were determined from the extrema in $B_{N}$. The $t_{D F B}$ was the duration of the enhanced $B_{N}$ region. The dipolarization fronts had mean $\Delta t$ of $1.1 s$ and mean $\Delta B_{N}$ of $20.9 n T$, which was comparable to the values obtained in the closer planet tail regions (Sundberg et al., 2012) ( $X_{M S M}^{\prime}$ was from $\sim-1.8$ to $-2.0 R_{M}$ in this study, and was from -1.5 to $-1 R_{M}$ in Sundberg et al. (2012)). Mean $\overline{t_{D F B}}$ was $2.1 s$ which was smaller than the values in the closer planet region. Figures $8 \mathrm{e}$ and $8 \mathrm{f}$ also showed that the $\Delta t$ and the $\Delta B_{N}$, the $\Delta t$ and the $t_{D F B}$ were positively correlated, which indicated that the larger scale of the dipolarization fronts $(\Delta t)$, the stronger the amplitudes $\left(\Delta B_{N}\right)$ and the larger scales of the DFB $\left(t_{D F B}\right)$.

Similar to the flux rope analysis on the CME event, we can use the background average Alfvén speed in the plasma sheet to estimate the scale of the dipolarization fronts and the DFBs. The average $B_{t}$ is $24 \mathrm{nT}$ from 22:34:00 to 22:42:00 UTC. After considering the densities of proton, $\mathrm{He}^{++}$, and $\mathrm{Na}^{+}$, the Alfvén speed is calculated to be 400 $\mathrm{km} / \mathrm{s}$. Therefore, the dipolarization fronts have an average scale of $440 \mathrm{~km}$, and the DFBs are $840 \mathrm{~km}$. Proton inertial length in the plasma sheet is estimated to be $240 \mathrm{~km}$, which indicates that both the dipolarization fronts and the DFBs are ion-scale. 

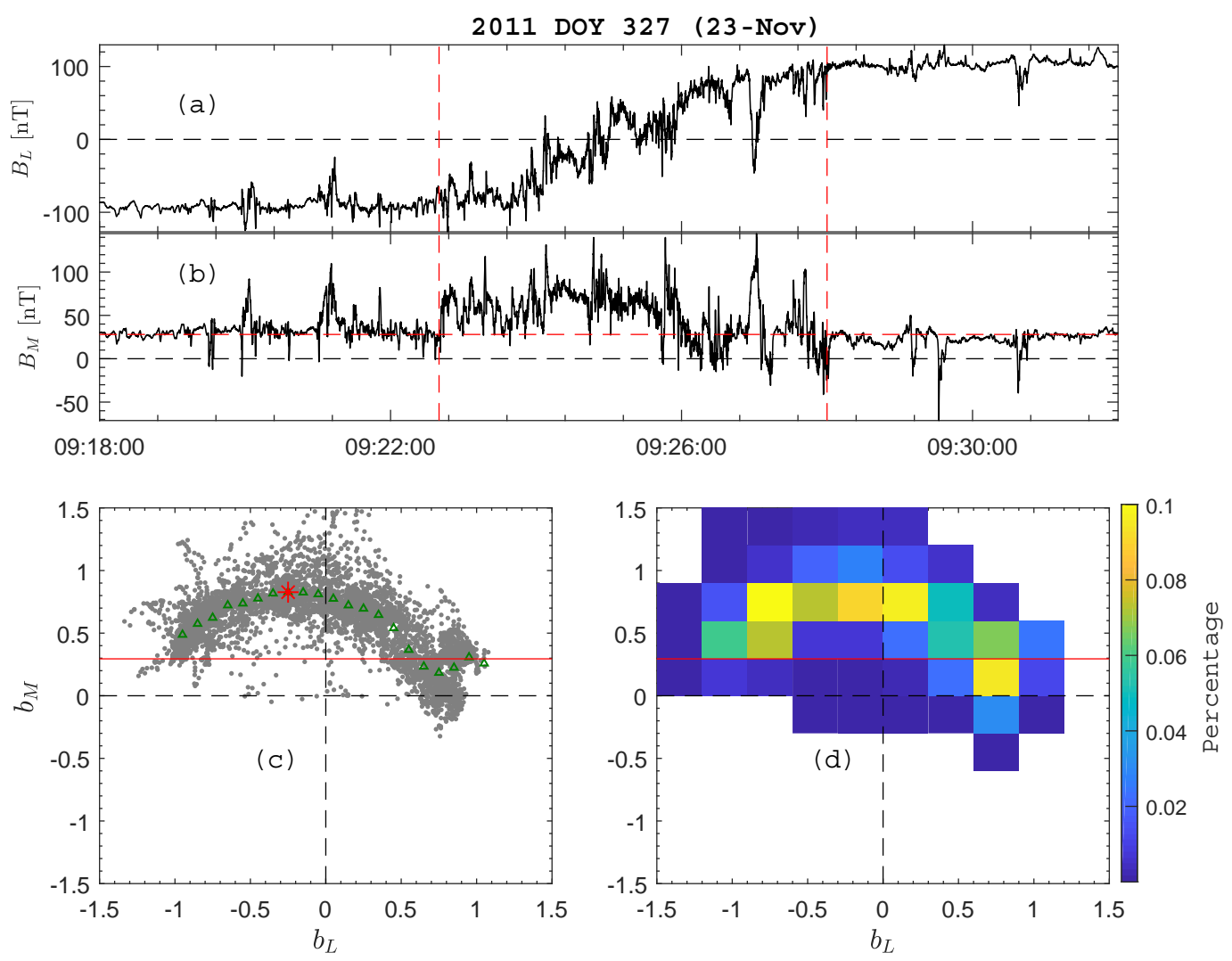

Figure 9. Analysis of the out-of-plane magnetic field component $\left(B_{M}\right)$ in the plasma sheet on 23 November 2011. (a) $B_{L}$, (b) $B_{M}$, (c) $b_{L}$ versus $b_{M}, b_{L}$ is $B_{L} / B_{\text {Lobe }}, b_{M}$ is $B_{M} / B_{\text {Lobe }}$. Green triangles indicate the averages of $b_{M}$ in $0.1 b_{L}$ bins. The red asterisk corresponds to the maximum $b_{M}$. (d) colormap for $b_{L}$ versus $b_{M}$, color represent the percentage of data points in each bin.

\subsection{Reconnection Features in the Plasma Sheet on 23 November 2011 CME}

\subsubsection{Hall Magnetic Field in Magnetic Reconnection with Strong Guide Field}

Figure 9 shows the analysis of the out-of-plane magnetic field component $\left(B_{M}\right)$ in the plasma sheet on 23 November 2011. The spacecraft entered the plasma sheet at $\sim$ 09:22:40 UTC from the southern lobe (the first vertical dashed line in Figures 9a and $9 \mathrm{~b}$ ) when $\left|B_{L}\right|$ (Figure 9a) and the magnetic field intensity started to decrease (Figure $4 \mathrm{e}$ ) and proton flux started to enhance (Figure 4a). The spacecraft traveled northward and moved out of the plasma sheet into the northern lobe at $\sim$ 09:28:00 UTC (the second vertical dashed line in Figures $9 \mathrm{a}$ to $9 \mathrm{~b}$ ) when $B_{L}$ became stable and positive and proton flux decreased. The red horizontal dashed line in Figure $9 \mathrm{~b}$ represents the $B_{\text {guide }}$, which was determined from the $B_{M}$ averaged between 09:18:00 UTC and 09:19:00 UTC in the southern lobe. The intensity of $B_{\text {guide }}$ was $\sim 28.0 n T$, which was $\sim 0.29$ when normalize to $B_{\text {Lobe }}(\sim 95.0 n T)$. It can be seen that $B_{M}$ were generally along the red horizontal dashed line in the southern and northern lobes, implying that the guide field was stable without large variations during this period. 
The $B_{M}$ (Figure $9 \mathrm{~b}$ ) in the plasma sheet with a vertical scale of $\sim 0.20 R_{M}$ showed an increase first and then a decrease relative to the guide field, which indicated a crossing of quadrupole Hall magnetic field associated with magnetic reconnection (Sonnerup, 1979). However, the $B_{M}$ was asymmetric comparing to the guide field with most of the $B_{M}$ being larger than the $B_{\text {guide }}$. In Figures $9 \mathrm{c}$ and $9 \mathrm{~d}, b_{M}$ (the $B_{M}$ normalized to the $\left.B_{\text {Lobe }}, B_{M} / B_{\text {Lobe }}\right)$ was shown as a function of $b_{L}\left(B_{L} / B_{\text {Lobe }}\right)$, in which the measurements in the flux ropes were excluded. The $b_{M}$ was asymmetric relative to the guide field (horizontal red line) and the largest $b_{M}$ was $\sim 1$, which means that the largest $B_{M}$ was comparable to the $B_{\text {Lobe }}$. Most of the $b_{M}$ was larger than the $B_{\text {guide }}$ and only a small portion of $b_{M}$ was smaller than the $B_{\text {guide }}$, which was concentrated on the region where the $b_{L}$ was larger than 0.5 corresponding to the outer part of the northern plasma sheet. In Figure $9 \mathrm{c}$, the green triangles were averaged $b_{M}$ in 0.1 bins of $b_{L}$. The maximum $b_{M}$ of green triangles was $\sim 0.83$ when $b_{L}$ was $-0.25 \pm 0.05$. The $b_{M}$ started to become lower than $0.29\left(B_{\text {guide }} / B_{\text {Lobe }}\right)$ when $b_{L}$ was larger than $0.65 \pm 0.05$ (0.05 is the half width of the bin). The minimum $b_{M}$ was $\sim 0.184$ when $b_{L}$ was $0.75 \pm 0.05$.

The analysis in Section 3.2 has shown that flux ropes continuously appeared implying that magnetic reconnection kept on occurring, and MESSENGER crossed the tailward of the NMNL in most of the times. This observation of enhanced $B_{M}$ region (positive perturbation) was much wider than weaken $B_{M}$ region (negative perturbation) suggesting a distorted pattern of the quadrupole Hall magnetic field, which could be generated by magnetic reconnection with a strong guide field. Several studies demonstrate that the Lorentz force could displace electron motion in the current sheet normal direction and cause asymmetrical Hall currents and therefore, distorts the quadrupole magnetic field in the magnetic reconnection region (Pritchett \& Coroniti, 2004; Huba, 2005; Eastwood et al., 2010). Consequently, the region of Hall magnetic field in the same direction of the guide field would be enlarged and the other region with opposite directed Hall magnetic field would be shrink (a scenario shown in Figure 10a). Huba (2005) predicted that when the guide field became larger than 0.34, the quadrupole Hall magnetic field profile would be eliminated and the Hall magnetic field would only point in one direction. In this case, the $B_{\text {guide }} / B_{\text {Lobe }}$ was $\sim 0.29$, only a small portion of Hall magnetic field was observed to be negative ( $\sim 3.3 \%$ of the data points), which was consistent with this conclusion.

In Figures $9 \mathrm{c}$ and $9 \mathrm{~d}$, a few $b_{M}$ grey points were smaller than the guide field in the southern part of the plasma sheet $\left(b_{L}<0\right)$. This arose from the situation that when flux rope passed over the spacecraft the leading part of the tailward traveling flux ropes contained the negative Hall magnetic field perturbations (a scenario shown in Figure 10b). In Figure 5, the region ahead of the leading flux ropes $(\sim$ 09:21:10 UTC and $\sim$ 09:24:47 UTC) contained periods of $B_{M}$ smaller than $B_{\text {guide }}(28 \mathrm{nT})$ corresponding to this scenario.

\subsubsection{Reconnection Rate}

Figure 11 shows the Harris current sheet fitting on the cross-tail current sheet and the $B_{N}$ inside the current sheet. The one-dimensional Harris current sheet model (Harris, 1962) is:

$$
B_{L}(z)=B_{L B} \tanh \left(\frac{z-z_{0}}{L_{c s}}\right)
$$

, where $B_{L}$ is the $L$ component of magnetic field in the magnetotail, $B_{L B}$ is lobe magnetic field intensity, $L_{C S}$ is half thickness of current sheet, $z$ is position of each $B_{L}$ measurement, $z_{0}$ is position of current sheet center. We employed a similar procedure as Sun et al. (2017) in the fitting. A parameter $\chi^{2}$ is introduced: 
(a)

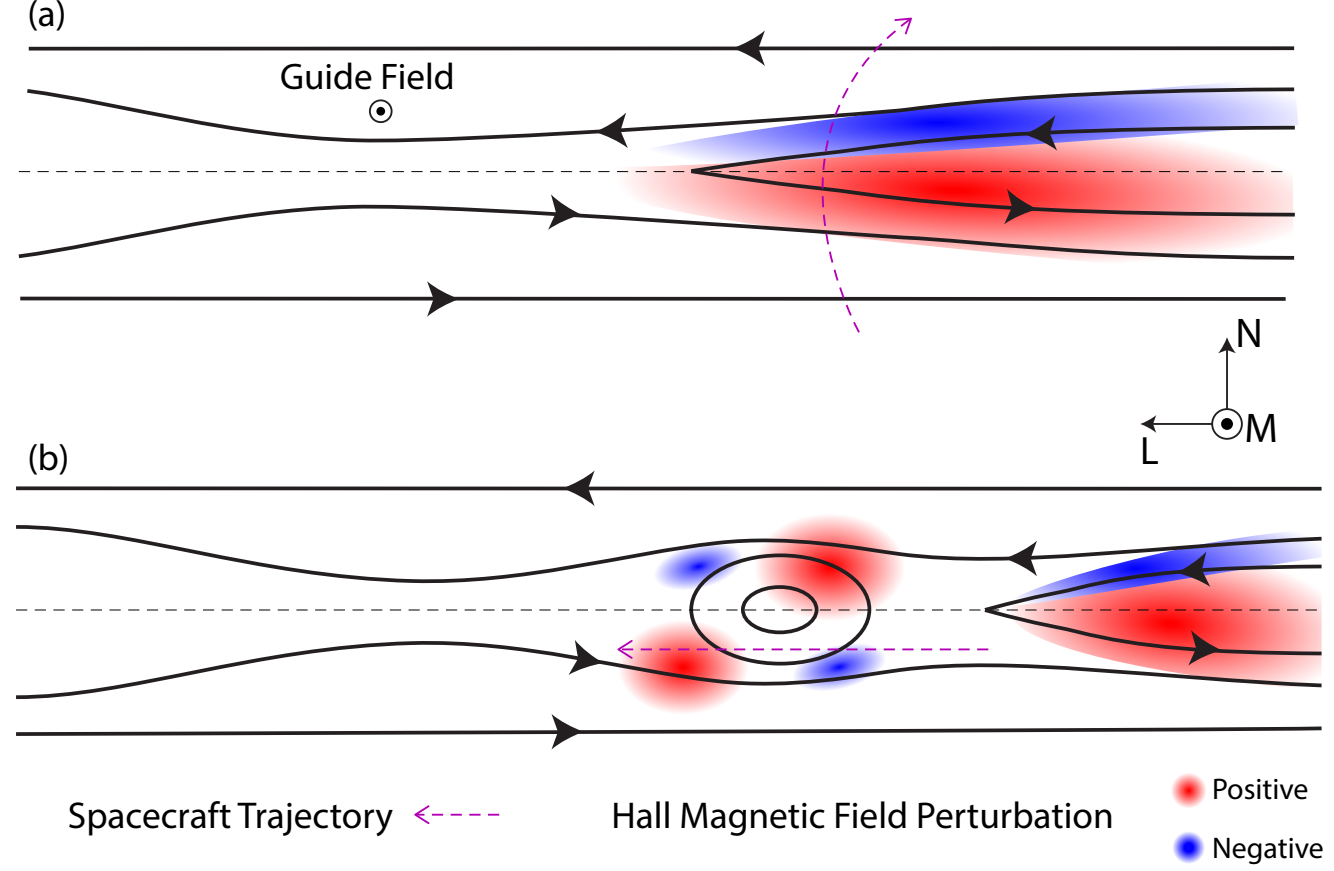

Figure 10. Schematic of the Hall magnetic field and Hall current associated with the guide field magnetic reconnection in the plasma sheet. (a), guide field magnetic reconnection with a single X-line. (b), the situation when a magnetic flux rope was formed.

$$
\chi^{2}=\frac{1}{N_{\text {point }}} \sum_{i=1}^{N_{\text {point }}}\left(\frac{\left|B_{L}^{H C S}(i)-B_{L}(i)\right|}{B_{L}^{H C S}(i)}\right)^{2}
$$

, in which $N_{\text {point }}$ is number of data points, $B_{L H C S}$ is magnetic field resulted from the Harris current sheet model, $B_{L}$ is measured $L$ component of magnetic field. The $B_{L}$ was averaged in a $40 \mathrm{~s}$ sliding window prior to the fitting to remove field fluctuations. In Figure 11a, blue curve is fitted $B_{L}$ from the Harris current sheet model, which is similar to the measured magnetic field shown in black. In the fitting, the $\chi^{2}$ is $\sim 1.22 \times$ $10^{-3}$ indicating a good fit. The $B_{L B}$ is $95.0 \mathrm{nT}$ and $L_{C S}$ is $0.046 R_{M}$. Current density $\left(J_{M}\right)$ resulted from the Harris current sheet model is shown in Figure 11b, and the maximum current density is $\sim 670 \mathrm{nA} / \mathrm{m}^{2}$. Average current sheet parameters in Mercury's tail (Poh et al., 2017a, 2017b) have a lobe magnetic field intensity of $\sim 41.0 \mathrm{nT}$, half thickness of $\sim 0.19 R_{M}$, and cross-tail current density of $\sim 92 \mathrm{nA} / \mathrm{m}^{2}$. This cross-tail current sheet had much thinner thickness, stronger current density and larger lobe field intensity. As shown in section 3.2, the gyroradius estimating from the thermal temperature is $\sim 100 \mathrm{~km}$. This value was comparable to the half thickness of the current sheet $\left(L_{C S}, 112 \mathrm{~km}\right)$, which indicates that majority of protons should undergo meandering motion in the current sheet.

The dimensionless magnetic reconnection rate could be calculated in several ways, including the ratio of reconnection inflow velocity to outflow velocity, the aspect ratio of reconnection diffusion region, normalized out-of-plane electric field, and the ratio of normal magnetic field component to reconnecting magnetic field in inflow region (Sonnerup, 1974; Sonnerup et al., 1981; Cassak \& Fuselier, 2016). MESSENGER could not directly resolve reconnection-associated plasma flows and did not provide measurements of elec- 
2011 DOY 327 (23-Nov)

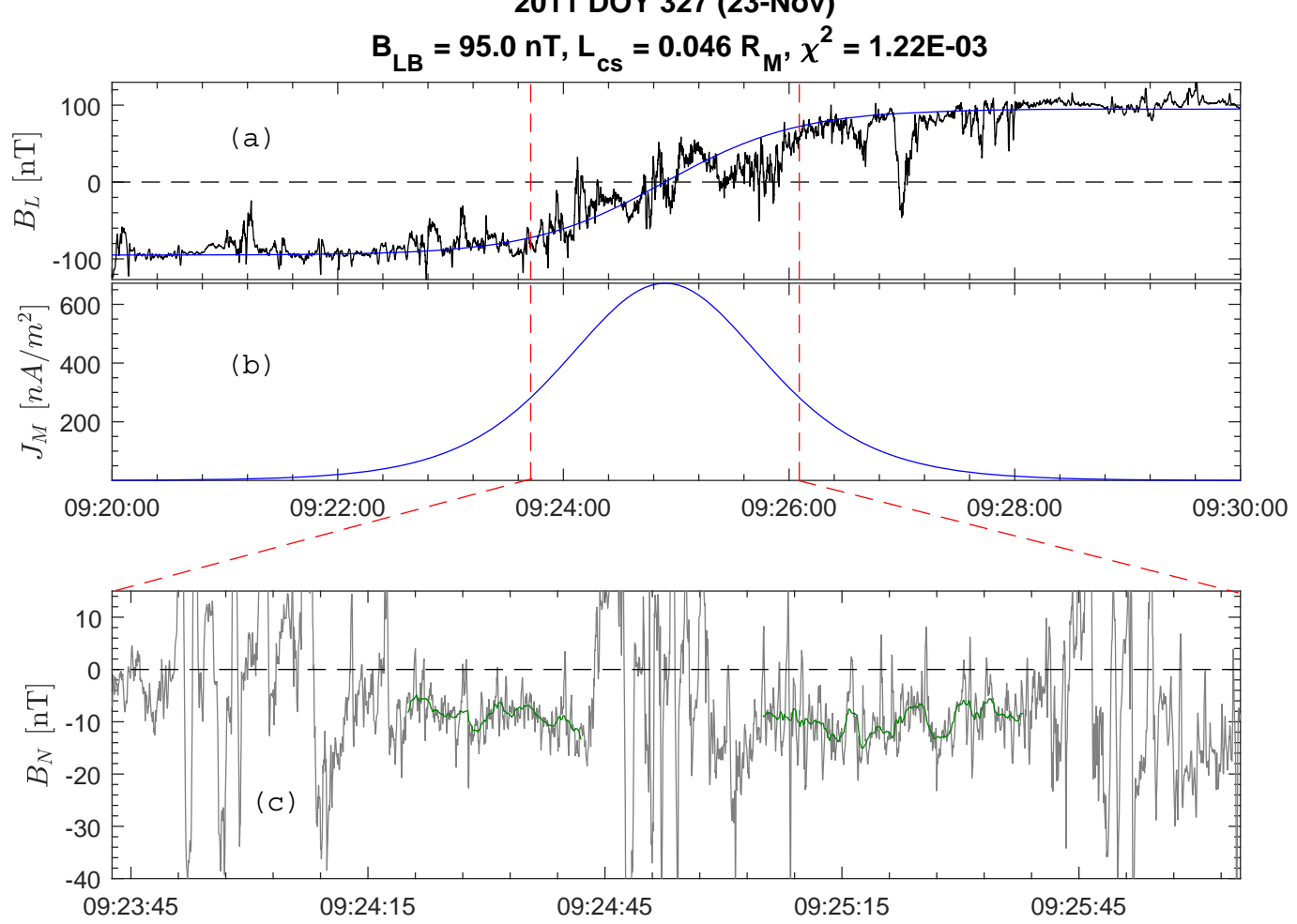

Figure 11. Harris current sheet fit on the cross-tail current sheet and the $B_{N}$ in the current sheet. (a) $B_{L}$, black line is from the measurements, blue line is from the fitting of Harris current sheet model. (b) $J_{M}$, the current density in $M$ direction obtained from Harris current sheet model. (c) $B_{N}$ in the current sheet. Green line is the sliding average of $B_{N}$ in $2 \mathrm{~s}$. 
tric fields. Therefore, we employ the ratio of normal magnetic field component to the lobe reconnecting magnetic field $\left(B_{N} / B_{L B}\right)$ to calculate the dimensionless reconnection rate, similar to previous MESSENGER studies (e.g., DiBraccio et al., 2013; Slavin et al., 2014; Zhong et al., 2018). Figure 11c shows the $B_{N}$ inside the current sheet. A large number of flux ropes showed up in the current sheet. To obtain the $B_{N}$ of the magnetic reconnection, the duration of the large scale flux ropes should be excluded. Two durations marked by the green lines contain relatively stable $B_{N}$ were selected. They were the prolonged negative $B_{N}$ in two flux rope groups, which started at 09:24:08 UTC and the 09:24:47 UTC. The green lines are the sliding average of $B_{N}$ in $2 \mathrm{~s}$. The average $B_{N}$ from the two durations was $\sim-8.85 \pm 2.4 n T$, where $2.4 n T$ is one standard deviation. Because the $B_{L B}$ was $95.0 \mathrm{nT}$, the dimensionless reconnection rate $\left(R_{M R}\right)$ was calculated to be $\sim$ $0.093 \pm 0.025$. However, the $B_{N}$ showed perturbations in the current sheet, and the flux ropes were frequently observed, which was suggested to be able to modulate the reconnection rate (e.g., Karimabadi et al., 2007). The value of $\sim 0.093$ should be an average dimensionless magnetic reconnection rate in this plasma sheet.

We performed the similar analysis of the out-of-plane and normal magnetic field components in the plasma sheet on the 11 May 2012 HSS event (not shown here). We did not see clear Hall magnetic field pattern as observed on 23 November 2011. This could be due to several reasons. In the plasma sheet on the 11 May 2012 HSS event (Figure 7), large number of dipolarization fronts appeared. First of all, the strong field-aligned currents associated with the dipolarization fronts, which was revealed in the Earth's study (J. Liu et al., 2013; Sun et al., 2013), could influence the out-of-plane magnetic field component. Secondly, the plasma sheet crossing was $\sim 0.6 R_{M}$ planetward of the NMNL as determined in Section 3.3. The reconnection-generated magnetic structures, mostly dipolarization fronts, could be largely influenced by the dipole field during their planetward travelling and would deform the reconnected fields.

\section{Southern Lobe Observations}

\subsection{Lobe Magnetic Field on 23 November 2011 CME}

The magnetic field measurements in the magnetotail on 23 November 2011 (the CME event) are shown in Figure 12 (black lines). As a comparison, the magnetic field intensity in the neighboring magnetotail crossing on 22 November 2011 from $\sim$ 20:00:00 to 21:50:00 UTC are shown in blue dashed lines in Figure 12d, which represent the magnetic field intensity of an average magnetotail.

We analyze the lobe region between $\sim$ 08:45:00 UTC and $\sim$ 09:19:00 UTC for the CME event. The lobe region contained many prominent plasma filaments before 08:45:00 UTC, which will be further discussed in Section 4.3., and the spacecraft started to enter the plasma sheet after 09:19:00 UTC. There were full of small-amplitude $B_{t}$ peaks during this period. The $B_{t}$ peaks became more prominent when MESSENGER closer to the plasma sheet. They were identified as TCRs, which last few seconds and contains asymmetric bipolar in $B_{z}$ and enhancements in $B_{x}$ and $B_{t}$. The TCRs should associate with the flux ropes in the plasma sheet and the magnetopause. Magnetic field measurements in blue dashed lines do not show these many $B_{t}$ peaks confirming a relatively quiet magnetotail.

Other than the small scale plasma filaments and TCRs, the magnetic field intensity was steady with an average value of $\sim 92.8 \pm 4.8 n T\left(B_{\text {Lobe }}\right)$ (4.8 nT was one standard deviation) without signatures of magnetic flux loading-unloading. Magnetic field line elevation angle is calculated from

$$
\theta_{B}=\arctan \left(\frac{\sqrt{B_{y}^{2}+B_{z}^{2}}}{\left|B_{x}\right|}\right)
$$


2011 DOY 327 (23-Nov)

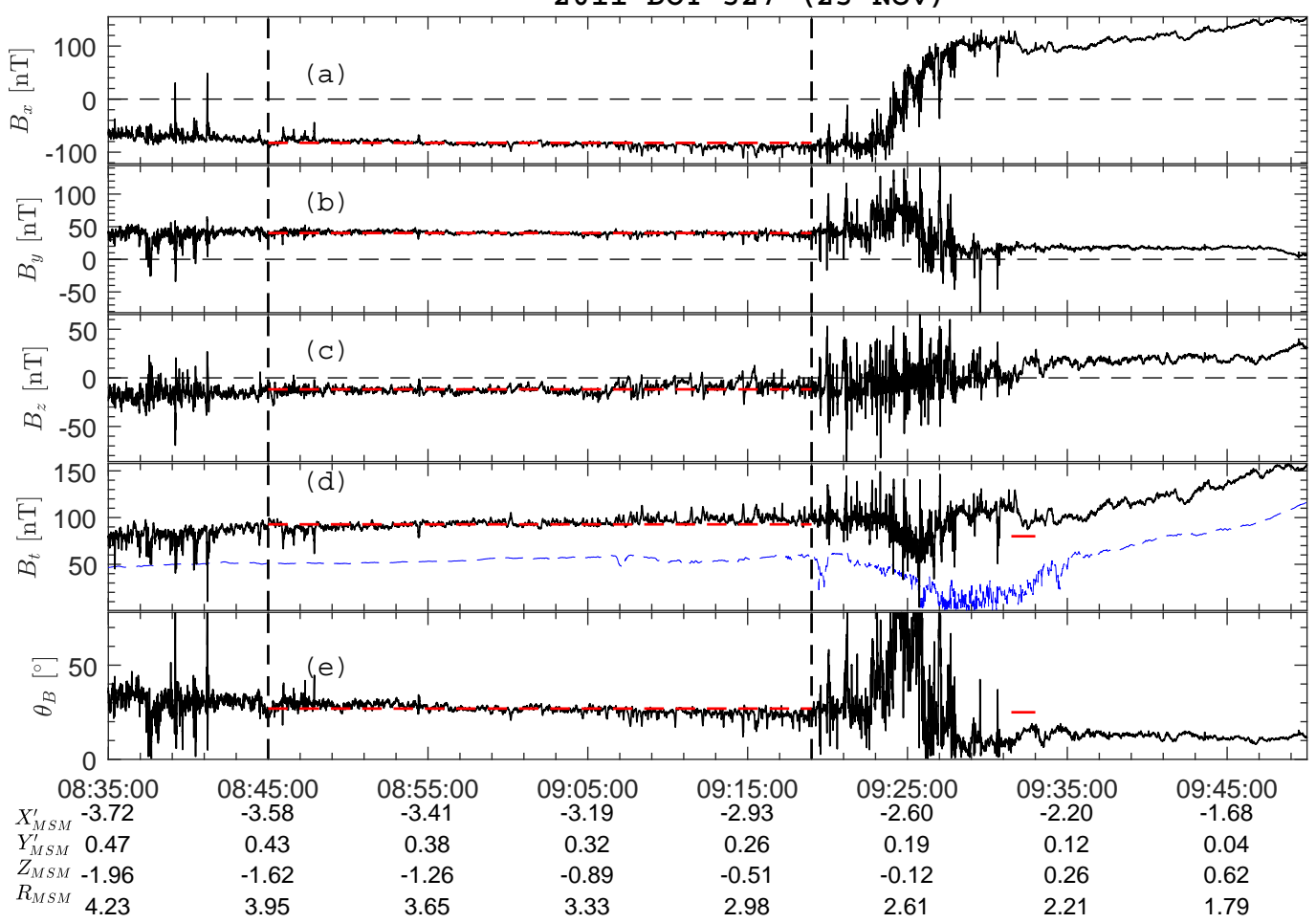

Figure 12. Overview of the magnetic field measurements in the magnetotail from 08:35:00 UTC to 09:50:00 UTC on 23 November 2011 during the CME (Black lines). (a) $B_{x}$. (b) $B_{y}$. (c) $B_{z}$. (d) magnetic field intensity $\left(B_{t}\right)$. (e) magnetic field line elevation angle $\left(\theta_{B}\right)$. Blue dashed line in (d) represent the measurements from the neighboring magnetotail crossing on 22 November 2011 prior to the CME impact. Red dashed horizontal lines represent the averages of each quantity in the period between the two vertical black dashed lines in the southern lobe. The red ticks in (d) and (e) mark a magnetic field decrease at 09:32:00 UTC in the northern lobe. 
, which is shown in Figure 12e. It was stable confirming that the magnetic field lines was steady without signatures of magnetotail reconfiguration. Mercury's magnetosphere was under the impact of a CME. The IMF was observed to be southward before MESSENGER crossed the tail magnetopause. The average magnetic field intensity in the lobe was more than twice the average magnetic field intensity $(\sim 41 n T)$ in Mercury's lobe at downtail distance of $\sim 3.5 R_{M}$ (Slavin, Anderson, et al., 2012; Poh et al., 2017b), and highfrequency reconnection-related TCRs were observed throughout the lobe. Also, the plasma sheet contained continuous flux ropes and negative $B_{N}$. All these features demonstrated that the magnetotail was extremely active. The time duration between the two vertical dashed lines ( $\sim 34$ minutes) in Figure 12 was a lower limit for the preserving of this feature, which corresponded to a duration of more than ten Dungey Cycles at Mercury (a mean value of $\sim 195$ second) (Slavin et al., 2010; Sun et al., 2015; Imber \& Slavin, 2017).

When MESSENGER entered into the northern lobe, there was one clear magnetic field decrease from 09:31:30 UTC to 09:33:00 UTC as marked by the red ticks in Figures $12 \mathrm{~d}$ and $12 \mathrm{e}$. This magnetic field decrease did not correspond to $\theta_{B}$ decrease but increase, which was likely caused by total pressure decrease outside the magnetosphere but not a magnetic flux unloading (see, Imber \& Slavin, 2017). After this magnetic field decrease, the total magnetic field gradually increase accompanying with decrease of $\theta_{B}$, which should be contributed by the dipole magnetic field as MESSENGER getting closer to the planet.

Based on the above features, we concluded that Mercury's magnetosphere was under the quasi-steady convection, in which the rates of magnetic flux into and out of the magnetotail should be comparable. This quasi-steady convection perhaps analogous to the steady magnetospheric convection (SMC) at Earth (Pytte et al., 1978), or possibly the continuous magnetospheric dissipation (CMD) studied by Tanskanen et al. (2005).

The open flux content of the tail lobe $\left(\Phi_{\text {Lobe }}\right)$ in this event is calculated according to the expression,

$$
\Phi_{\text {Lobe }}=B_{\text {Lobe }}\left(\frac{\pi R_{\text {Tail }}^{2}}{2}-d_{c s} R_{\text {Tail }}\right)
$$

, where $B_{\text {Lobe }}$ is the average magnetic field intensity in the lobe, $R_{\text {Tail }}$ is the magnetotail radius, $d_{c s}$ is the thickness of the cross-tail current sheet. The cross-sectional area of one hemisphere of Mercury's tail was calculated from $R_{\text {Tail }}$ by assuming that the magnetotail was a semicircle. Subtracting the half cross-sectional area of the plasma sheet, in which the plasma sheet was assumed to be a rectangle, the cross-sectional area of the lobe could be obtained. Multiplying the $B_{\text {Lobe }}$ and the cross-sectional area of the lobe obtained the open flux content of the lobe $\left(\Phi_{\text {Lobe }}\right)$. The $B_{\text {Lobe }}$ was assumed to be uniform in the lobe, which was $\sim 92.8 n T$, and the steady magnetic field measurements in Figure 12 consisted with this assumption. The $R_{\text {Tail }}$ was determined to be $\sim 2.23 R_{M}$ in section 2.2. The $d_{c s}$ was obtained through Harris current sheet fitting on the crosstail current sheet, which was $\sim 0.092 R_{M}$ as shown in Figure 11.

The $\Phi_{\text {Lobe }}$ was calculated to be $\sim 4.20 \mathrm{MWb}$, which was much higher than the mean open flux content in the lobe $(\sim 62 \%$ higher than the 2.6 MWb in Johnson et al. (2012) and $\sim 68 \%$ higher than the $2.5 \mathrm{MWb}$ in Imber and Slavin (2017)). In Figure 12d, the magnetic field intensity in the CME event (the black line) is significantly larger than (almost twice) the average magnetotail (the blue dashed line), which is consistent with the conclusion that the CME event contains extreme large open flux. Using the dipole moment of $190 n T \cdot R_{M}^{3}$ (Anderson et al., 2012), the magnetic flux closed outside Mercury's surface was $\sim 7.25 \mathrm{MWb}$. This value is obtained through integrating the magnetic field in the magnetic equatorial plane outside the $\sim 0.98 R_{M}$. The $0.98 R_{M}$ corresponds to Mercury's surface in the magnetic equatorial plane, which was obtained based on the northward offset of Mercury's dipole $\left(\sim 0.2 R_{M}\right)$ from the center of the planet. The amount of magnetic flux in the southern lobe $(\sim 4.2 \mathrm{MWb})$ implied that $\sim 56 \%$ of the magnetic 
2012 DOY 132 (11-May)

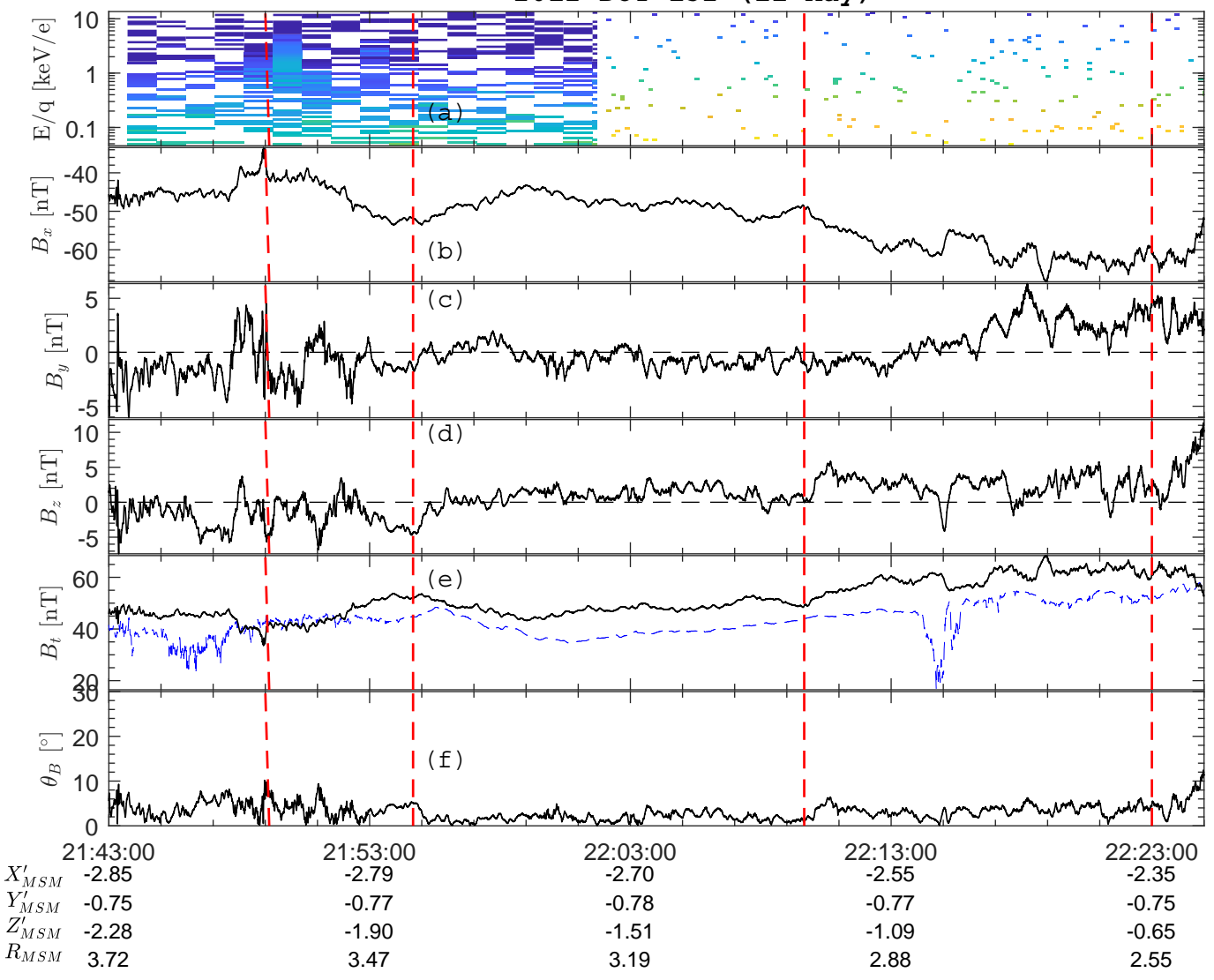

Figure 13. Overview of the proton and magnetic field measurements in the southern lobe from 21:43:00 UTC to 22:25:00 UTC on 11 May 2012 during the HSS impact (black lines). (a) Proton differential particle flux. (b) $B_{x}$, (c) $B_{y}$, (d) $B_{z}$, (e) $B_{t}$, (f) magnetic field line elevation angle $\theta_{B}$. Blue dashed lines in each panel represent the measurements from the neighboring magnetotail crossing prior to the HSS impact. Red vertical dashed lines represent the duration of magnetic field increases.

flux in Mercury's magnetosphere was open. However, because this event is during the impact of a CME, the studies of Slavin et al. (2014) and (Jia et al., 2019) show that magnetic flux contributed by induction currents in Mercury's interior cannot be neglected. They determine that the effective magnetic moment for Mercury during this CME impact was $\sim 216 n T \cdot R_{M}^{3}$. The total magnetic flux closed outside Mercury's surface is then calculated to be $\sim 8.25 \mathrm{MWb}$. The $\sim 4.2 \mathrm{MWb}$ corresponded to $\sim 51 \%$ of the total magnetic flux. On the other hand, the open flux in the lobe $(\sim 4.2 \mathrm{MWb})$ during the steady convection is $\sim 42.4 \%$ larger than the maximum open magnetic flux during Mercury's Dungey cycle ( 2.95 $M W b)$ (Imber \& Slavin, 2017).

For comparison, the magnetic flux closed outside Earth's surface was $\sim 8 G W b$ (Milan et al., 2004), the polar cap open flux was smaller than $1 \mathrm{GWb}(\sim 12.5 \%)$ even during the intense substorms (AE > $1000 \mathrm{nT}$ ) (Petrinec \& Russell, 1996; Milan et al., 2004; DeJong et al., 2007) and the open flux for SMCs and isolated substorms were comparable (DeJong et al., 2007; Tanskanen et al., 2005). 


\subsection{Lobe Magnetic Field on 11 May 2012 HSS}

Proton and magnetic field measurements in the southern lobes on 11 May 2012 are displayed in Figure 13 black lines. Magnetic field intensity from the neighboring magnetotail crossing on the same day from 13:30:00 UTC to 15:00:00 UTC are shown as blue dashed line in Figure 13e. The magnetic field intensity (Figure 13e) for the HSS event was not as steady as the lobe magnetic field intensity on the CME event (Figure 12d). We identified magnetic field enhancements with duration $>30 s$ in both cases. Two magnetic field intensity enhancements were identified on 11 May 2012 as shown in Figure 13. The first enhancement between the first and second vertical dashed lines was actually a magnetic depression comparing with the surroundings. The magnetic depression was possibly caused by a diamagnetic effect, in which particle flux was enhanced in the leading part (around the first vertical dashed line). The second enhancement between the third and fourth vertical dashed lines did not correspond to clear $\theta_{B}$ increase but slightly decrease, which was likely a consequence of total pressure enhancement outside Mercury's magnetosphere (see, Imber \& Slavin, 2017) but was not a magnetic flux loading. Further, as can be seen in Figure 7f, magnetic field intensity increased after MESSENGER crossed the current sheet ( 22:45:00 UTC) comparing with the magnetic field intensity before the current sheet crossing $(\sim 22: 30: 00$ UTC). This feature is similar to the CME event, which is because MESSENGER getting closer to the planet and the dipole magnetic field becoming stronger. Therefore, no clear magnetic flux loading was observed in the lobe 11 May 2012 ( 42 minutes) and Mercury's magnetosphere was under the steady convection. The supplementary material provides magnetic field measurements on the northern lobe for the HSS event.

On 11 May 2012, the $B_{\text {Lobe }}$ was $\sim 59.1 n T$, which was averaged over 22:05:00 UTC to 22:23:00 UTC and the radius of magnetotail was $2.43 R_{M}$ (determined in Section 2.3). Assuming that thickness of the plasma sheet was $0.1 R_{M}$, the lobe open flux was calculated to be $\sim 3.2 \mathrm{MWb}$, which indicated that $43.8 \%$ of the planet's magnetic flux was open. The lobe open flux was $\sim 27.2 \%$ larger than the average value of $2.5 \mathrm{MWb}$, and was $\sim 7.8 \%$ larger than the maximum open magnetic flux $(\sim 2.95 \mathrm{MWb})$ during Mercury's Dungey cycle (Imber \& Slavin, 2017). In Figure 13e, the magnetic field intensity (blue dashed line) during the neighboring magnetotail crossing is much smaller $(\sim 40 n T)$ than the HSS event, consisting with that the HSS event contains much larger lobe open flux than the average magnetotail.

\subsection{Plasma Mantle on 23 November 2011 CME}

The proton and magnetic field measurements of the plasma mantle on 23 November 2011 and the calculation of cross-polar cap potential (CPCP) are shown in Figure 14. Following the crossing of the tail magnetopause (the first vertical dashed blue line, $\sim$ 08:28:00 UTC), the proton flux (Figure 14b) continuously decreased from $\sim 3 \times 10^{8} \mathrm{~cm}^{-2} \mathrm{~s}^{-1}$ to $\sim 6 \times 10^{6} \mathrm{~cm}^{-2} \mathrm{~s}^{-1}$ (the second vertical dashed blue line, $\sim$ 08:57:00 UTC), accompanying with a dispersion in the proton dynamic spectra (Figure 14a). The spectra show energy dispersion with the upper bound of the proton energy decreasing as distance from the tail magnetopause increases, which is the main feature of plasma mantle. The plasma in the mantle has characteristics similar to the magnetosheath plasma as expected (see, DiBraccio, Slavin, Raines, et al., 2015; Jasinski et al., 2017). The region of plasma mantle close to the tail magnetopause from $\sim$ 08:05:00 UTC to $\sim$ 08:42:00 UTC frequently observed discrete diamagnetic field decreases or increases, i.e., the plasma filaments and the TCRs. Those plasma filaments are the magnetospheric extensions of FTEs (Slavin et al., 2014; Poh et al., 2016), which contain magnetosheath plasma as is evident in Figure 14 .

Measurements in plasma mantle could be used to estimate the cross-magnetosphere electric field and then the CPCP. The calculation of the cross-magnetosphere electric field 
MESSENGER MAG FIPS 2011 DOY 327 , (23-Nov)

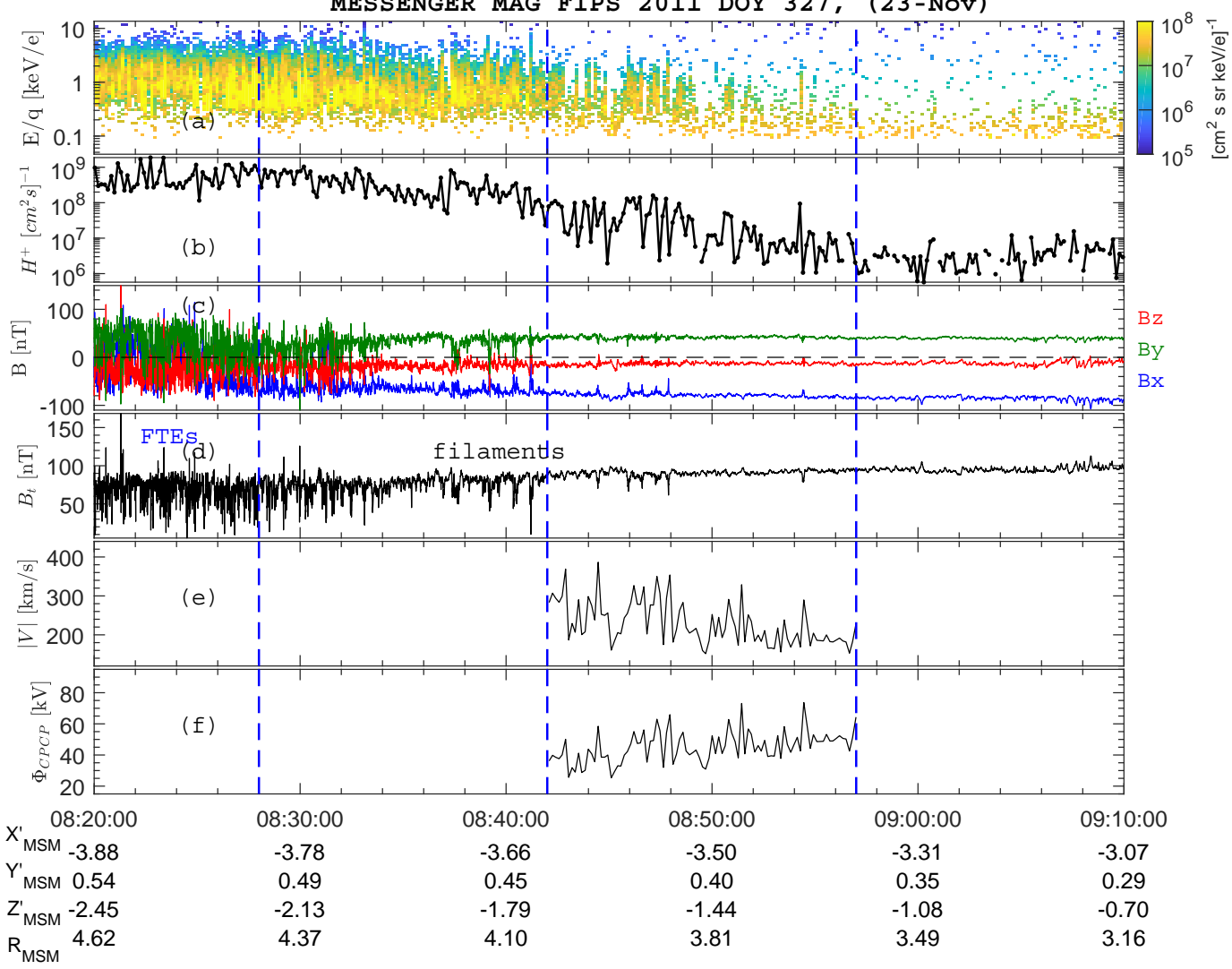

Figure 14. The proton and magnetic field measurements of the plasma mantle and calculation of the cross-polar cap potential (CPCP) from 08:20:00 UTC to 09:10:00 UTC on 23 November 2011. (a) Proton dynamic spectra. (b) Proton particle flux integrated over the FIPS energy range $(\sim 46 \mathrm{eV}$ to $\sim 13.3 \mathrm{keV})$. (c) $B_{x}$ (blue), $B_{y}$ (green), $B_{z}$ (red). (d) Magnetic field intensity $\left(B_{t}\right)$, FTEs are the flux transfer events. (e) Proton bulk velocities $(V)$. (f) the values of CPCP. The first vertical dashed blue line indicates the average magnetopause location. The second and third vertical dashed lines mark the start and end times of the calculations of CPCP.
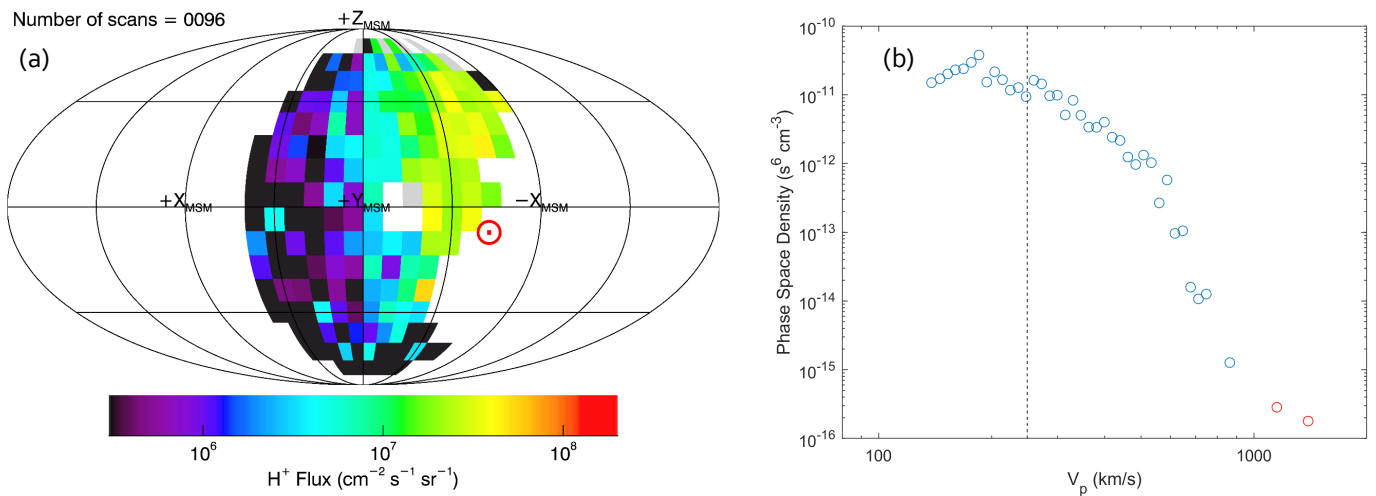

Figure 15. Proton distributions from FIPS in the plasma mantle from 08:42:00 UTC to 08:57:00 UTC on 23 November 2011. (a) Accumulated three-dimensional proton distribution in the MSM coordinate. The red circle indicate the magnetic field direction. (b) Averaged phase space density versus proton thermal velocity. The vertical dashed line represent the average thermal velocity of the observed protons. 
requires proton bulk velocity $(V)$ and dispersion edge angle $(\Theta)$ of the plasma mantle. The proton bulk velocities ( $V$, Figure 14e) were obtained from the FIPS measurements. The dispersion wedge angle $\left(\Theta_{\text {dispersion }}\right)$ of the plasma mantle could be determined from the distances between the observation point and the location of magnetopause $(\sigma)$, and the observation point and the terminator $(L)$, which was based on the assumption that the plasma mantle was originated at the terminator $\left(X_{M S M}^{\prime}=0\right)$. We calculated the $\left|V_{E \times B}\right|$ from $V \sin \Theta_{\text {dispersion }}$, and then we used $\left|V_{E \times B}\right| B$ to calculate the cross-magnetosphere electric field, and $\Phi_{C P C P}$ (Figure 14f) from $\left|V_{E \times B}\right| B d_{\text {Tail }}$, where $d_{\text {Tail }}$ was the width of magnetotail in dawn-dusk direction and was determined to be $\sim 4.46 R_{M}$. This calculation process was adopted from DiBraccio, Slavin, Raines, et al. (2015). However, we only performed these calculations to the plasma mantle portion deeper in the magnetosphere to mitigate the influences from FTEs (from $~$ 08:42:00 UTC to $\sim$ 08:57:00 UTC, Figures $14 \mathrm{e}$ to $14 \mathrm{f})$. The average magnetic field during this period was $[-80.7,40.7,-13.4]$ $\mathrm{nT}$. The cross-magnetosphere electric field in the plasma mantle was averaged to be $4.58 \pm$ $1.0 \mathrm{mV} / \mathrm{m}$ (duskward), and the $\Phi_{C P C P}$ was $45.1 \pm 9.8 \mathrm{kV}$. The $1.0 \mathrm{mV} / \mathrm{s}$ and $9.8 \mathrm{kV}$ are the standard deviations. This potential value was almost triple the average value of $\sim 16 \mathrm{kV}$ of Mercury's magnetosphere (Jasinski et al., 2017), which further confirmed that Mercury's nightside magnetosphere was under extreme driving from the solar wind.

The accumulated three-dimensional proton distribution from FIPS in the plasma mantle (from $\sim$ 08:42:00 UTC to $\sim$ 08:57:00 UTC) is shown in Figure 15. The angular map (Figure 15a) showed the integrated proton flux in the MSM coordinate, in which the direction of the magnetic field was located near the edge of the field-of-view of FIPS. The protons peaked around the magnetic field direction, which closed to the anti-sunward direction, and there were fewer particles in the directions further away from the magnetic field direction. This observation suggested that the protons were mostly moving antisunward, which was consistent with the flow pattern in the plasma mantle. The average proton phase space density from $\sim$ 08:42:00 UTC to $\sim$ 08:57:00 UTC was shown in Figure 15b, in which the average weighted velocity was determined to be $\sim 249 \mathrm{~km} / \mathrm{s}$.

Several uncertainties arose during the calculation of the CPCP, which include that the original position of plasma mantle could be away from the terminator, $|V|$ estimated from the FIPS might be affected by the field-of-view limitation, and the actual width of the magnetopause was unknown (DiBraccio, Slavin, Raines, et al., 2015). We note that the twist of magnetotail could also influence the calculation. The magnetotail often tilted towards dawn or dusk due to the non-zero Y (dawn-dusk) component in the IMF (Cowley, 1981; Owen et al., 1995). The average IMF outside the magnetopause had a large $Y$ component in this event as shown in Section 2.2, which might cause a tilt of the tail. In observations, deviation of the cross-tail current sheet normal from $\hat{z}_{M S M}^{\prime}$ might be used to indicate the overall twist of the magnetotail. The cross-tail current sheet normal, which was $\mathrm{N}=(-0.14,-0.07,0.99)$ (from Section 3.1 ), had a tilt angle of $\sim 4.0^{\circ}$ and the southern lobe tilts towards the dusk and the northern lobe tilts towards the dawn, which should only have a small influence on the calculations. Therefore, we ignored this effect in the calculation.

\section{Discussion}

\subsection{Response to the Dayside Magnetosphere Variations}

Slavin et al. (2014) studied the same CME and HSS impacts on Mercury's dayside magnetosphere as we consider here, but they only analyzed the dayside interaction. The solar wind dynamic pressures are similar for these two periods $(\sim 50 \mathrm{nPa})$. The CME event produces low $\beta(\sim 0.06)$ and thick plasma depletion layer in the magnetosheath, which leads to high reconnection rate despite of a small magnetic shear angle across the magnetopause $\left(\sim 60^{\circ}\right)$. The HSS event produces a relatively high $\beta$ magnetosheath but has a large shear angle $\left(\sim 160^{\circ}\right)$. The net effect of the high $\beta$ and large shear angle, which 
were opposing effects, is a lower dayside magnetopause reconnection rate for the HSS than the value for the CME. This assessment is supported by the deeper and broader cusp confirming the stronger magnetopause reconnection during the CME impact (Slavin et al., 2014).

In this study of the nightside magnetosphere, the lobe open flux was $\sim 32.1 \%$ higher during the CME event than the lobe open flux in the HSS event. The occurrence rate of flux ropes during the CME impact observed tailward of the NMNL was twice that of dipolarization fronts observed planetward of the NMNL during the HSS. Mercury's nightside magnetosphere during the CME event was clearly more active than during the HSS. These features strongly suggest that the magnetosheath $\beta$ controlled the magnetospheric activity in the tail. The low magnetosheath $\beta$ could produce strong plasma depletion layer at the dayside magnetopause and make the magnetopause reconnection rate independent of the magnetic shear angles (Sonnerup, 1974; Scurry et al., 1994). In this manner, the higher reconnection rate of the magnetopause appears to larger magnetic flux transfer from the dayside magnetosphere to the nightside magnetosphere, resulting in enhanced reconnection in the tail current sheets.

\subsection{Steady Convection in Mercury's magnetosphere}

On 23 November 2011, the lobe magnetic field intensity was steady and no clear magnetic flux loading and unloading events were observed, which sustained a period of at least ten Mercury's Dungey cycles ( 34 minutes) (Section 4.1). On 11 May 2012, the lobe also did not contain clear magnetic flux loading and unloading in $\sim 40$ minutes (Section 4.2). They both demonstrated that magnetic flux transfer rates in and out of the magnetotail were comparable on timescales of at least ten Dungey cycles for these two intense solar wind events. Therefore, Mercury's magnetosphere appears to have been under quasi-steady convection possibly in analogous to the SMC in Earth's magnetosphere. In Slavin, Anderson, et al. (2012), the authors analyzed another possible steady convection event during the second Mercury's flyby by MESSENGER. In that event, the IMF was steady southward and the IMF intensity was close to the average value in Mercury's orbit. The continuous appearance of flux ropes and TCRs suggested that the magnetic reconnection kept on occurring in the magnetotail without clear magnetic flux loadingunloading in $\sim 20$ minutes ( $\sim 7$ Mercury's Dungey cycles). The NMNL was determined to be located at $\sim X_{M S M}^{\prime} \sim-2.8 R_{M}$ (Slavin, Anderson, et al., 2012). The lobe magnetic field was $\sim 37 \mathrm{nT}\left(X_{M S M}^{\prime} \sim-3 R_{M}\right)$ and the open flux content was calculated to be $\sim 2.46 \mathrm{MWb}$ with tail radius of $2.7 R_{M}$ and plasma sheet thickness of $0.1 R_{M}$.

Table 2 summaries the location of the NMNL, the lobe open flux, and the solar wind condition for the steady convection events on 6 October 2008, 23 November 2011 and 11 May 2012. The steady convection on 6 October 2008 corresponded to the average solar wind condition. The steady convection events on 23 November 2011 and 11 May 2012 corresponded to extreme solar wind driving (a CME and a HSS). The lobe open flux on 6 October 2008 was $\sim 2.46 \mathrm{MWb}$, which was similar to the average lobe open flux $(\sim$ $2.5 \mathrm{MWb}$ ), and the location of the NMNL was $\sim-2.8 R_{M}$ also similar to the average location of NMNL (Poh et al., 2017b; Slavin, Anderson, et al., 2012). The lobe open flux was $\sim 4.2 \mathrm{MWb}$ and $\sim 3.2 \mathrm{MWb}$ on 23 November 2011 and 11 May 2012, which was $~$ $68 \%$ and $\sim 27.2 \%$ larger than the average lobe open flux, respectively. The locations of the NMNL in both events were closer to the planet than that on 6 October 2008.

There are several key aspects of SMC events in Earth's magnetosphere. Firstly, SMC events at Earth are associated with steady solar wind long period of southward IMF (> 5 to 10 hours) but only a modest negative IMF $B_{z}$ such as produces isolated substorms (O'Brien et al., 2002). Secondly, the lobe open flux during the SMC events (0.6 GWb) was comparable to the average lobe open flux and was slightly smaller than during the isolated substorms (DeJong et al., 2007; Milan et al., 2004). Thirdly, the near-Earth neu- 
tral line (NENL) was believed to be located in the mid-tail region at $>$ tens of $R_{E}$ (Sergeev et al., 1996; Yang et al., 2010). These aspects are also summarized in Table 2. The steady convection on 6 October 2008 in Mercury's magnetosphere (Slavin, Anderson, et al., 2012) was similar to the first two aspects in Earth's magnetosphere. It happened during a steady and long period of southward IMF and the lobe open flux $(\sim 2.46 \mathrm{MWb})$ was smaller than the maximum lobe open flux during Mercury's Dungey cycle ( $\sim 2.95 \mathrm{MWb})$. However, the NMNL was located at $\sim 2.8 R_{M}$ downtail, which corresponds to $22.4 R_{E}$ if one took a scaling factor of $\sim 8$ from Mercury to Earth (Siscoe et al., 1975), which was much closer to the planet than that at Earth. The steady convection events during the CME and the HSS at Mercury were different from SMC events in Earth's magnetosphere in all the three aspects. First of all, the steady convection event occurred under the impact of a CME or a HSS, which are extreme solar wind conditions. Hence, Mercury's magnetosphere was under much stronger solar wind driving than the SMC events in Earth's magnetosphere. Secondly, the lobe open flux was $\sim 68 \%$ and $\sim 27.2 \%$ larger than the average lobe open flux. Thirdly, the locations the NMNL was much closer to the planet than that in Earth's magnetosphere.

The above analysis suggested several unique features of the steady convection events in Mercury's magnetosphere. Firstly, the locations of NMNL were relatively closer to the planet than the locations of NENL during Earth's SMC. The closer NMNL locations to the planet could be a consequence of solar wind driving and the absence of steady ring current. On one hand, Mercury is closer to the Sun, corresponding to a lower solar wind Alfvén Mach number $(<5)$ than those of Earth $(\sim 7-10)$. Flux pileup and plasma depletion are commonly observed in front of the dayside magnetopause (Gershman et al., 2013), which would produce a low plasma $\beta$ environment and cause high dimensionless reconnection rate (Slavin \& Holzer, 1979; Slavin et al., 2009; DiBraccio et al., 2013). Comparing to Earth's dayside magnetopause, magnetic field intensity $\left(B_{S H}\right)$ is stronger and Alfvén speed $\left(V_{A S H}\right)$ is faster in the magnetosheath adjacent to Mercury's dayside magnetopause. Magnetic flux was transferred in a continuous manner during the steady convection, magnetotail reconnection needed to balance the high reconnection rate $\left(R_{M R} V_{A S H} B_{S H}\right)$ of the dayside magnetopause. Therefore, the magnetic reconnection needed to occur in the closer planet tail region, since the lobe field intensity was stronger and Alfvén speed was faster than those in the downtail region. On the other hand, the ring current in Earth's magnetosphere could enhance the dipole magnetic field in the downtail region, which would push reconnection further downtail. Because of the absence of a steady ring current in Mercury's magnetosphere, this effect was eliminated and could result in reconnection closer to the planet.

Secondly, steady convection event could happen in average solar wind condition (the observation on 6 October 2008) and extreme solar wind condition (CME impact on 23 November 2011 and HSS impact on 11 May 2012) in Mercury's magnetosphere. However, the SMC events are observed during average and steady solar wind conditions in Earth's magnetosphere (O'Brien et al., 2002; Partamies et al., 2009). In Earth's magnetosphere, a sawtooth event containing quasi-periodic magnetic flux loading-unloading in the lobe and energetic particle injections in the geosynchronous orbit is observed under the impact of a CME (see., Huang et al., 2003; Henderson et al., 2006).

The absence of ionosphere and inner magnetosphere in Mercury's magnetosphere might account for this unique property. In Earth's magnetosphere, ionospheric outflows would be enhanced during strong solar wind driving (e.g., Lennartsson \& Shelley, 1986; Moore et al., 1999; Echer et al., 2008). On one hand, as suggested by Brambles et al. (2011), ionospheric outflows could fill the inner magnetosphere, and then distend the nightside magnetic field line, which could push the X-line downward and resulted in quasiperiodic substorms. On the other hand, ionospheric outflows in the plasma sheet could slow the reconnection rate (Shay \& Swisdak, 2004; Zhang et al., 2016). Magnetic flux would be piled up in the lobe causing magnetic flux loading and push reconnection site 
tailward moving. As a consequence, a closer planet reconnection occurs and release the loaded magnetic flux resulting in unloading, and would also eventually cause quasi-periodic substorms, that is sawtooth event. However, Mercury's magnetosphere does not experience these influences from the inner magnetosphere and the ionosphere.

Thirdly, the steady magnetospheric convection at Mercury seems does not strongly depend on the polarity of IMF. In the CME event, the magnetic shear angle was $\left(\sim 117^{\circ}\right)$ before MESSENGER entered the tail magnetopause. On the dayside magnetopause, the magnetic shear angle became much smaller $\left(\sim 60^{\circ}\right)$, however, the plasma $\beta$ was low $(\sim$ 0.06) (Slavin et al., 2014). This suggests that the steady magnetospheric convection does not strongly rely on the magnetic shear angle, which should be due to the fact that the formation of thick plasma depletion layer, especially during the CME event, make the dayside magnetopause reconnection occur regardless of the magnetic shear angle (DiBraccio et al., 2013; Slavin et al., 2014; Scurry et al., 1994). This feature is different from the Earth's SMC, which requires weak negative $B_{z}$ (O'Brien et al., 2002; Partamies et al., 2009).

\subsection{Dawn-Dusk Extent of Magnetic Reconnection in the Plasma Sheet on 23 November 2011}

During the steady convection, the magnetic flux transported to the plasma sheet from lobes should be equal to the magnetic flux transported by reconnection outflows. On 23 November 2011, the CPCP was determined to be $\sim 45.1 \mathrm{kV}$ (shown in Section 4.3) on the basis of our analysis of FIPS measurements in the high-latitude mantle. Given this fact, the dawn-dusk extent of the tail magnetic reconnection could be easily derived. This implies a magnetic flux transport rate from the lobe to the cross-tail current sheet of $45.1 \mathrm{kWb} / \mathrm{s}$. The speed of reconnection outflow should be the Alfvén speed in the inflow region $\left(V_{A L}\right)$, which was calculated to be $\sim 2090 \mathrm{~km} / \mathrm{s}$ based on a density of $1.01 \mathrm{~cm}^{-3}$ and the $B_{L B}$ of $95 \mathrm{nT}$. Magnetic flux transported by the magnetic reconnection in the plasma sheet can be calculated through $R_{M R} V_{A L} B_{L B} Y_{\text {extent }}$, where $R_{M R}$ was the dimensionless reconnection rate, $Y_{\text {extent }}$ was the extent of X-line in the dawn-dusk direction. The average $R_{M R}$ was determined to $\sim 0.093$ (Section 3.4). Therefore, the $Y_{\text {extent }}$ was $\sim 2441 \mathrm{~km}\left(\sim 1 R_{M}\right)$. This indicates that $\sim 20.7 \%$ of the cross-tail current sheet needs to reconnect to balance the transport of magnetic flux in Mercury's magnetotail. The X-line dawn-dusk extent obtained here should be an average value. The variations of the magnetic reconnection rate would result in changes in the X-line extent. Table 3 summarizes the features of magnetic reconnection in the plasma sheet on 23 November 2011 , including the guide field, maximum $b_{M}$, reconnection rate and dawn-dusk extent of the X-line.

\section{Conclusion}

This study investigated and compared the dynamics of Mercury's nightside magnetosphere during the impact of a CME and a HSS. Our analysis of Mercury's magnetotail and the comparisons with tail dynamics in Earth's magnetosphere resulted in several important conclusions.

(1) The CME on 23 November 2011 produced quasi-periodic flux rope groups with a mean duration of 70 seconds on the tailward side of the NMNL. The flux rope groups contained large-scale flux ropes (tens of proton inertial lengths) in the leading part followed by smaller-scale flux ropes (several proton inertial lengths). The HSS on 11 May 2012 produced dipolarization fronts on the planetward side of the NMNL. These reconnectiongenerated magnetic structures are separated by only several seconds and they had length scales comparable with a proton inertial length. The occurrence rate for the flux ropes and dipolarization fronts are two orders of magnitude higher than the occurrence rate averaged over all plasma sheet observations (Sun et al., 2016). 
(2) The open magnetic flux in the tail lobes during these extreme solar wind events was around half of the Mercury's total available magnetic flux, i.e., 58\% for the CME and $\sim 44 \%$ for the HSS. These open magnetic flux are also much larger than the maximum lobe open magnetic flux during Mercury's Dungey cycle, i.e., $\sim 42 \%$ for the CME and $\sim 7.8 \%$ for the HSS.

(3) The occurrence rate of the reconnection-generated magnetic structures during low $\beta$ magnetosheath CME event is twice that during the high $\beta$ magnetosheath HSS event. Further, the lobe open magnetic flux during the CME event (4.2 MWb) is much larger than that $(3.2 \mathrm{MWb})$ during the HSS event. These results suggest that enhanced reconnection due to low magnetosheath $\beta$ may lead directly to more reconnection in Mercury's tail consistent with the suggestions of earlier studies (Slavin et al., 2014; DiBraccio et al., 2013).

(4) In the CME event, magnetic reconnection produces a distorted Hall magnetic field (the out-of-plane component) pattern in the plasma sheet. The MESSENGER measurements suggests a strong guide field $\left(B_{\text {guide }} / B_{\text {Lobe }} \sim 0.29\right)$. The cross magnetosphere potential drop $(45 \mathrm{kV})$ is around three times the average value $(15 \mathrm{kV})$, and the dawndusk extend of the X-line corresponds to $20 \%$ of the tail width.

(5) No tail lobe magnetic flux loading and unloading events similar to substorms or sawtooth events were observed suggesting that rates of magnetic flux into and out of the magnetotail were similar on time scales at least ten Mercury's Dungey cycles (half an hour) during these CME and HSS events. Mercury's nightside magnetosphere was under a type of quasi-steady convection during these extreme solar wind conditions. Together with previous observation by Slavin, Anderson, et al. (2012), the quasi-steady convection could occur over a wide range of solar wind conditions in Mercury's magnetosphere. SMC events at Earth require the steady solar wind magnetic field and velocity of average intensity (O'Brien et al., 2002). The relative locations of the NMNL during quasi-steady convection events at Mercury was much closer to the planet than at Earth based upon the scaling between Mercury and Earth. We suggest that the lack of an inner magnetosphere and an ionosphere, which could influence the tail reconnection during extreme conditions, makes the steady convection possible at Mercury. The low solar wind Alfvén Mach number and the lack of steady ring current account for the relatively closer location of the NMNL at Mercury. 


\section{Acknowledgments}

MESSENGER data used in this study were available from the Planetary Data System (PDS): http://pds.jpl.nasa.gov. The MESSENGER project was supported by the NASA Discovery Program under contracts NASW-00002 to the Carnegie Institution of Washington and NAS5-97271 to The Johns Hopkins University Applied Physics Laboratory. WJS and JAS were supported by NASA grants NNX16AJ67G and 80NSSC18K1137. JMR was supported by NASA Discovery Data Analysis grant NNX15AE77G. WJS thanks to Dr. Lihui Chai for helpful discussions.

\section{References}

Akasofu, S.-I. (1964). The development of the auroral substorm. Planetary and Space Science, 12(4), 273 - 282. Retrieved from http:// www.sciencedirect.com/science/article/pii/0032063364901515 doi: https://doi.org/10.1016/0032-0633(64)90151-5

Akhavan-Tafti, M., Slavin, J. A., Le, G., Eastwood, J. P., Strangeway, R. J., Russell, C. T., .. Burch, J. L. (2018). Mms examination of ftes at the earth's subsolar magnetopause. Journal of Geophysical Research: Space Physics, 123(2), 12241241. Retrieved from https://agupubs.onlinelibrary.wiley.com/doi/abs/ 10.1002/2017JA024681 doi: 10.1002/2017JA024681

Alexeev, I. I., Belenkaya, E. S., Yu. Bobrovnikov, S., Slavin, J. A., \& Sarantos, M. (2008). Paraboloid model of mercury's magnetosphere. Journal of Geophysical Research: Space Physics, 113(A12). Retrieved from https:// agupubs.onlinelibrary.wiley.com/doi/abs/10.1029/2008JA013368 doi: 10.1029/2008JA013368

Anderson, B. J., Acuña, M. H., Korth, H., Slavin, J. A., Uno, H., Johnson, C. L., ... McNutt, R. L. (2010, May 01). The magnetic field of mercury. Space Science Reviews, 152(1), 307-339. Retrieved from https://doi.org/10.1007/ s11214-009-9544-3 doi: 10.1007/s11214-009-9544-3

Anderson, B. J., Acuña, M. H., Lohr, D. A., Scheifele, J., Raval, A., Korth, H., \& Slavin, J. A. (2007, Aug 01). The magnetometer instrument on MESSENGER. Space Science Reviews, 131(1), 417-450. Retrieved from https://doi.org/ 10.1007/s11214-007-9246-7 doi: 10.1007/s11214-007-9246-7

Anderson, B. J., Johnson, C. L., Korth, H., Winslow, R. M., Borovsky, J. E., Purucker, M. E., .. McNutt Jr., R. L. (2012). Low-degree structure in mercury's planetary magnetic field. Journal of Geophysical Research: Planets, 117(E12). Retrieved from https://agupubs.onlinelibrary.wiley.com/doi/abs/ 10.1029/2012JE004159 doi: 10.1029/2012JE004159

Andrews, G. B., Zurbuchen, T. H., Mauk, B. H., Malcom, H., Fisk, L. A., Gloeckler, G., ... Raines, J. M. (2007, Aug 01). The energetic particle and plasma spectrometer instrument on the MESSENGER spacecraft. Space Science Reviews, 131(1), 523-556. Retrieved from https://doi.org/10.1007/ s11214-007-9272-5 doi: 10.1007/s11214-007-9272-5

Angelopoulos, V., Runov, A., Zhou, X.-Z., Turner, D. L., Kiehas, S. A., Li, S.S., \& Shinohara, I. (2013). Electromagnetic energy conversion at reconnection fronts. Science, 341(6153), 1478-1482. Retrieved from https://science.sciencemag.org/content/341/6153/1478 doi: $10.1126 /$ science. 1236992

Baker, D. N., Pulkkinen, T. I., Angelopoulos, V., Baumjohann, W., \& McPherron, R. L. (1996). Neutral line model of substorms: Past results and present view. Journal of Geophysical Research: Space Physics, 101 (A6), 12975-13010. Retrieved from https://agupubs.onlinelibrary.wiley.com/doi/abs/ 10.1029/95JA03753 doi: 10.1029/95JA03753

Belian, R. D., Cayton, T. E., \& Reeves, G. D. (1995). Quasi-periodic global substorm generated flux variations observed at geosynchronous orbit. 
. P. D. M. Ashour-Abdalla T. Chang (Ed.), Space plasmas: Coupling between small and medium scale processes (p. 143-148). C.: American Geophysical Union (AGU). Washington, D. 10.1029/GM086p0143

Brambles, O. J., Lotko, W., Zhang, B., Wiltberger, M., Lyon, J., \& Strangeway, R. J. (2011). Magnetosphere sawtooth oscillations induced by ionospheric outflow. Science, 332(6034), 1183-1186. Retrieved from https://science.sciencemag.org/content/332/6034/1183 doi: 10.1126/science.1202869

Cassak, P. A., \& Fuselier, S. A. (2016). Reconnection at Earth's Dayside Magnetopause. In W. Gonzalez \& E. Parker (Eds.), Magnetic reconnection: Concepts and applications (Vol. 427, p. 213-276). doi: 10.1007/978-3-319-26432-5_6

Chen, C. X., \& Wolf, R. A. (1999). Theory of thin-filament motion in earth's magnetotail and its application to bursty bulk flows. Journal of Geophysical Research: Space Physics, 104(A7), 14613-14626. Retrieved from https:// agupubs.onlinelibrary.wiley.com/doi/abs/10.1029/1999JA900005 doi: 10.1029/1999JA900005

Chen, Y., Tóth, G., Cassak, P., Jia, X., Gombosi, T. I., Slavin, J. A., .. Henderson, M. G. (2017). Global three-dimensional simulation of earth's dayside reconnection using a two-way coupled magnetohydrodynamics with embedded particle-in-cell model: Initial results. Journal of Geophysical Research: Space Physics, 122(10), 10,318-10,335. Retrieved from https:// agupubs.onlinelibrary.wiley.com/doi/abs/10.1002/2017JA024186 doi: 10.1002/2017JA024186

Chen, Y., Tóth, G., Jia, X., Slavin, J. A., Sun, W., Markidis, S., .. Raines, J. M. (n.d.). Studying dawn-dusk asymmetries of mercury's magnetotail using mhdepic simulations. Journal of Geophysical Research: Space Physics, n/a(n/a). Retrieved from https://agupubs.onlinelibrary.wiley.com/doi/abs/ 10.1029/2019JA026840 doi: 10.1029/2019JA026840

Cowley, S. (1981). Magnetospheric asymmetries associated with the y-component of the imf. Planetary and Space Science, 29(1), 79 - 96. Retrieved from http://www.sciencedirect. com/science/article/pii/0032063381901410 doi: https://doi.org/10.1016/0032-0633(81)90141-0

DeJong, A. D., Cai, X., Clauer, R. C., \& Spann, J. F. (2007). Aurora and open magnetic flux during isolated substorms, sawteeth, and smc events. Annales Geophysicae, 25(8), 1865-1876. Retrieved from https://www.ann-geophys .net/25/1865/2007/ doi: 10.5194/angeo-25-1865-2007

DeJong, A. D., Ridley, A. J., Cai, X., \& Clauer, C. R. (2009). A statistical study of bris (smcs), isolated substorms, and individual sawtooth injections. Journal of Geophysical Research: Space Physics, 114(A8). Retrieved from https:// agupubs.onlinelibrary.wiley.com/doi/abs/10.1029/2008JA013870 doi: 10.1029/2008JA013870

Dewey, R. M., Raines, J. M., Sun, W., Slavin, J. A., \& Poh, G. (2018). MESSENGER observations of fast plasma flows in mercury's magnetotail. Geophysical Research Letters, 45(19), 10,110-10,118. Retrieved from https:// agupubs.onlinelibrary.wiley.com/doi/abs/10.1029/2018GL079056 doi: 10.1029/2018GL079056

Dewey, R. M., Slavin, J. A., Raines, J. M., Baker, D. N., \& Lawrence, D. J. (2017). Energetic electron acceleration and injection during dipolarization events in mercury's magnetotail. Journal of Geophysical Research: Space Physics, 122(12), 12,170-12,188. Retrieved from https://agupubs.onlinelibrary .wiley.com/doi/abs/10.1002/2017JA024617 doi: 10.1002/2017JA024617

DiBraccio, G. A., Slavin, J. A., Boardsen, S. A., Anderson, B. J., Korth, H., Zurbuchen, T. H., ... Solomon, S. C. (2013). MESSENGER observa- 
tions of magnetopause structure and dynamics at mercury. Geophysical Research: Space Physics, 118(3), 997-1008. https://agupubs.onlinelibrary.wiley.com/doi/abs/10.1002/jgra.50123 doi: 10.1002/jgra.50123

DiBraccio, G. A., Slavin, J. A., Imber, S. M., Gershman, D. J., Raines, J. M., Jackman, C. M., ... Solomon, S. C. (2015). MESSENGER observations of flux ropes in mercury's magnetotail. Planetary and Space Science, 115, 77 - 89. Retrieved from http://www.sciencedirect.com/science/article/pii/ S0032063314004085 (Solar wind interaction with the terrestrial planets) doi: https://doi.org/10.1016/j.pss.2014.12.016

DiBraccio, G. A., Slavin, J. A., Raines, J. M., Gershman, D. J., Tracy, P. J., Boardsen, S. A., ... Solomon, S. C. (2015). First observations of mercury's plasma mantle by MESSENGER. Geophysical Research Letters, 42(22), 9666-9675. Retrieved from https://agupubs.onlinelibrary.wiley.com/doi/abs/ 10.1002/2015GL065805 doi: 10.1002/2015GL065805

Dungey, J. W. (1961, Jan). Interplanetary magnetic field and the auroral zones. Phys. Rev. Lett., 6, 47-48. Retrieved from https://link.aps.org/doi/ 10.1103/PhysRevLett.6.47 doi: 10.1103/PhysRevLett.6.47

Eastwood, J. P., Shay, M. A., Phan, T. D., \& Øieroset, M. (2010, May). Asymmetry of the ion diffusion region hall electric and magnetic fields during guide field reconnection: Observations and comparison with simulations. Phys. Rev. Lett., 104, 205001. Retrieved from https://link.aps.org/doi/10.1103/ PhysRevLett.104.205001 doi: 10.1103/PhysRevLett.104.205001

Echer, E., Korth, A., Zong, Q.-G., Fraünz, M., Gonzalez, W. D., Guarnieri, F. L., ... Reme, H. (2008). Cluster observations of o+ escape in the magnetotail due to shock compression effects during the initial phase of the magnetic storm on 17 august 2001. Journal of Geophysical Research: Space Physics, 113(A5). Retrieved from https://agupubs.onlinelibrary.wiley.com/doi/abs/ 10.1029/2007 JA012624 doi: 10.1029/2007JA012624

Genestreti, K. J., Nakamura, T. K. M., Nakamura, R., Denton, R. E., Torbert, R. B., Burch, J. L., .. Russell, C. T. (2018). How accurately can we measure the reconnection rate em for the mms diffusion region event of 11 july 2017? Journal of Geophysical Research: Space Physics, 123(11), 9130-9149. Retrieved from https://agupubs.onlinelibrary.wiley.com/doi/abs/ 10.1029/2018JA025711 doi: 10.1029/2018JA025711

Gershman, D. J., Slavin, J. A., Raines, J. M., Zurbuchen, T. H., Anderson, B. J., Korth, H., ... Solomon, S. C. (2013). Magnetic flux pileup and plasma depletion in mercury's subsolar magnetosheath. Journal of Geophysical Research: Space Physics, 118(11), 7181-7199. Retrieved from https:// agupubs.onlinelibrary.wiley.com/doi/abs/10.1002/2013JA019244 doi: 10.1002/2013JA019244

Harris, E. G. (1962, Jan 01). On a plasma sheath separating regions of oppositely directed magnetic field. Il Nuovo Cimento (1955-1965), 23(1), 115-121. Retrieved from https://doi.org/10.1007/BF02733547 doi: 10.1007/BF02733547

Henderson, M. G., Reeves, G. D., Skoug, R., Thomsen, M. F., Denton, M. H., Mende, S. B., ... Singer, H. J. (2006). Magnetospheric and auroral activity during the 18 april 2002 sawtooth event. Journal of Geophysical Research: Space Physics, 111(A1). Retrieved from https://agupubs.onlinelibrary .wiley.com/doi/abs/10.1029/2005JA011111 doi: 10.1029/2005JA011111

Huang, C.-S., Foster, J. C., Reeves, G. D., Le, G., Frey, H. U., Pollock, C. J., \& Jahn, J.-M. (2003). Periodic magnetospheric substorms: Multiple space-based and ground-based instrumental observations. Journal of Geophysical Research: Space Physics, 108(A11). Retrieved from https:// agupubs.onlinelibrary.wiley.com/doi/abs/10.1029/2003JA009992 doi: 


\subsection{9/2003JA009992}

Huba, J. D. (2005). Hall magnetic reconnection: Guide field dependence. Physics of Plasmas, 12(1), 012322. 1.1834592 doi: $10.1063 / 1.1834592$

Imber, S. M., \& Slavin, J. A. (2017). MESSENGER observations of magnetotail loading and unloading: Implications for substorms at mercury. Journal of Geophysical Research: Space Physics, 122(11), 11,402-11,412. Retrieved from https://agupubs.onlinelibrary.wiley.com/doi/abs/10.1002/ 2017JA024332 doi: 10.1002/2017JA024332

Imber, S. M., Slavin, J. A., Auster, H. U., \& Angelopoulos, V. (2011). themis survey of flux ropes and traveling compression regions: Location of the near-earth reconnection site during solar minimum. Journal of Geophysical Research: Space Physics, 116(A2). Retrieved from https:// agupubs.onlinelibrary.wiley.com/doi/abs/10.1029/2010JA016026 doi: 10.1029/2010JA016026

Jasinski, J. M., Slavin, J. A., Raines, J. M., \& DiBraccio, G. A. ～(2017). Mercury's solar wind interaction as characterized by magnetospheric plasma mantle observations with MESSENGER. Journal of Geophysical Research: Space Physics, 122(12), 12,153-12,169. Retrieved from https:// agupubs.onlinelibrary.wiley.com/doi/abs/10.1002/2017JA024594 doi: 10.1002/2017JA024594

Jia, X., Slavin, J. A., Poh, G., DiBraccio, G. A., Toth, G., Chen, Y., ... Gombosi, T. I. (2019). MESSENGER observations and global simulations of highly compressed magnetosphere events at mercury. Journal of Geophysical Research: Space Physics, 124(1), 229-247. Retrieved from https:// agupubs.onlinelibrary.wiley.com/doi/abs/10.1029/2018JA026166 doi: 10.1029/2018JA026166

Johnson, C. L., Purucker, M. E., Korth, H., Anderson, B. J., Winslow, R. M., Al Asad, M. M. H., ... Solomon, S. C. (2012). MESSENGER observations of mercury's magnetic field structure. Journal of Geophysical Research: Planets, 117(E12). Retrieved from https://agupubs.onlinelibrary.wiley.com/doi/ abs/10.1029/2012JE004217 doi: 10.1029/2012JE004217

Karimabadi, H., Daughton, W., \& Scudder, J. (2007). Multi-scale structure of the electron diffusion region. Geophysical Research Letters, 34(13). Retrieved from https://agupubs.onlinelibrary.wiley.com/doi/abs/10.1029/ 2007GL030306 doi: 10.1029/2007GL030306

Lennartsson, W., \& Shelley, E. G. (1986). Survey of 0.1- to 16-kev/e plasma sheet ion composition. Journal of Geophysical Research: Space Physics, 91(A3), 3061-3076. Retrieved from https://agupubs.onlinelibrary.wiley.com/ doi/abs/10.1029/JA091iA03p03061 doi: 10.1029/JA091iA03p03061

Liu, J., Angelopoulos, V., Runov, A., \& Zhou, X.-Z. (2013). On the current sheets surrounding dipolarizing flux bundles in the magnetotail: The case for wedgelets. Journal of Geophysical Research: Space Physics, 118(5), 20002020. Retrieved from https://agupubs.onlinelibrary.wiley.com/doi/abs/ 10.1002/jgra.50092 doi: 10.1002/jgra.50092

Liu, Y.-H., Li, T. C., Hesse, M., Sun, W. J., Liu, J., Burch, J., .. Huang, K. (2019). Three-dimensional magnetic reconnection with a spatially confined x-line extent: Implications for dipolarizing flux bundles and the dawn-dusk asymmetry. Journal of Geophysical Research: Space Physics, 124(4), 28192830. Retrieved from https://agupubs.onlinelibrary.wiley.com/doi/abs/ 10.1029/2019JA026539 doi: 10.1029/2019JA026539

Ma, Z. W., Otto, A., \& Lee, L. C. (1994). Core magnetic field enhancement in single $\mathrm{x}$ line, multiple $\mathrm{x}$ line and patchy reconnection. Journal of Geophysical Research: Space Physics, 99(A4), 6125-6136. Retrieved from https : //agupubs . onlinelibrary.wiley.com/doi/abs/10.1029/93JA03480 
doi: 10.1029/93JA03480

McPherron, R. L., Russell, C. T., \& Aubry, M. P. (1973). Satellite studies of magnetospheric substorms on august 15, 1968: 9. phenomenological model for substorms. Journal of Geophysical Research (1896-1977), 78(16), 3131-3149. Retrieved from https://agupubs.onlinelibrary.wiley.com/doi/abs/ 10.1029/JA078i016p03131 doi: 10.1029/JA078i016p03131

Milan, S. E., Cowley, S. W. H., Lester, M., Wright, D. M., Slavin, J. A., Fillingim, M., ... Singer, H. J. (2004). Response of the magnetotail to changes in the open flux content of the magnetosphere. Journal of Geophysical Research: Space Physics, $109(\mathrm{~A} 4)$ Retrieved from https:// agupubs.onlinelibrary.wiley.com/doi/abs/10.1029/2003JA010350 doi: 10.1029/2003JA010350

Moore, T. E., Peterson, W. K., Russell, C. T., Chandler, M. O., Collier, M. R., Collin, H. L., ... Pollock, C. J. (1999). Ionospheric mass ejection in response to a cme. Geophysical Research Letters, 26(15), 2339-2342. Retrieved from https://agupubs.onlinelibrary.wiley.com/doi/abs/10.1029/ 1999GL900456 doi: 10.1029/1999GL900456

Nagai, T., Fujimoto, M., Saito, Y., Machida, S., Terasawa, T., Nakamura, R., ... Kokubun, S. (1998). Structure and dynamics of magnetic reconnection for substorm onsets with geotail observations. Journal of Geophysical Research: Space Physics, 103(A3), 4419-4440. Retrieved from https : //agupubs. onlinelibrary . wiley.com/doi/abs/10.1029/97JA02190 doi: 10.1029/97JA02190

Nakamura, T. K. M., Genestreti, K. J., Liu, Y.-H., Nakamura, R., Teh, W.-L., Hasegawa, H., ... Giles, B. L. (2018). Measurement of the magnetic reconnection rate in the earth's magnetotail. Journal of Geophysical Research: Space Physics, 123(11), 9150-9168. Retrieved from https:// agupubs.onlinelibrary.wiley.com/doi/abs/10.1029/2018JA025713 doi: 10.1029/2018JA025713

Ness, N. F., Behannon, K., Lepping, R., \& Whang, Y. (1976). Observations of mercury's magnetic field. Icarus, 28(4), 479 - 488. Retrieved from http://www.sciencedirect.com/science/article/pii/0019103576901214 doi: https://doi.org/10.1016/0019-1035(76)90121-4

Ness, N. F., Behannon, K. W., Lepping, R. P., Whang, Y. C., \& Schatten, K. H. (1974). Magnetic field observations near mercury: Preliminary results from mariner 10. Science, 185(4146), 151-160. Retrieved from https://science.sciencemag.org/content/185/4146/151 doi: 10.1126/science.185.4146.151

O'Brien, T. P., Thompson, S. M., \& McPherron, R. L. (2002). Steady magnetospheric convection: Statistical signatures in the solar wind and ae. Geophysical Research Letters, 29(7), 34-1-34-4. Retrieved from https:// agupubs.onlinelibrary.wiley.com/doi/abs/10.1029/2001GL014641 doi: 10.1029/2001GL014641

Ohtani, S.-i., Shay, M. A., \& Mukai, T. (2004). Temporal structure of the fast convective flow in the plasma sheet: Comparison between observations and twofluid simulations. Journal of Geophysical Research: Space Physics, 109(A3). Retrieved from https://agupubs.onlinelibrary.wiley.com/doi/abs/ 10.1029/2003JA010002 doi: 10.1029/2003JA010002

Owen, C. J., Slavin, J. A., Richardson, I. G., Murphy, N., \& Hynds, R. J. (1995) Average motion, structure and orientation of the distant magnetotail determined from remote sensing of the edge of the plasma sheet boundary layer with e i, $35 \mathrm{kev}$ ions. Journal of Geophysical Research: Space Physics, 100(A1), 185-204. Retrieved from https://agupubs.onlinelibrary.wiley .com/doi/abs/10.1029/94JA02417 doi: 10.1029/94JA02417

Partamies, N., Pulkkinen, T., McPherron, R., McWilliams, K., Bryant, C., Tan- 
skanen, E., ... Thomsen, M. (2009). Statistical survey on sawtooth events, smcs and isolated substorms. Advances in Space Research, 44(3), 376 - 384. Retrieved from http://www.sciencedirect.com/science/article/pii/ S0273117709001963 doi: https://doi.org/10.1016/j.asr.2009.03.013

Petrinec, S. M., \& Russell, C. T. (1996). Near-earth magnetotail shape and size as determined from the magnetopause flaring angle. Journal of Geophysical Research: Space Physics, $101(\mathrm{A1}), 137-152 . \quad$ Retrieved from https : //agupubs . onlinelibrary .wiley.com/doi/abs/10.1029/95JA02834 doi: 10.1029/95JA02834

Poh, G., Slavin, J. A., Jia, X., DiBraccio, G. A., Raines, J. M., Imber, S. M., ... Solomon, S. C. (2016). MESSENGER observations of cusp plasma filaments at mercury. Journal of Geophysical Research: Space Physics, 121(9), 82608285. Retrieved from https://agupubs.onlinelibrary.wiley.com/doi/abs/ 10.1002/2016JA022552 doi: 10.1002/2016JA022552

Poh, G., Slavin, J. A., Jia, X., Raines, J. M., Imber, S. M., Sun, W.-J., ... Smith, A. W. (2017a). Coupling between mercury and its nightside magnetosphere: Cross-tail current sheet asymmetry and substorm current wedge formation. Journal of Geophysical Research: Space Physics, 122(8), 8419-8433. Retrieved from https://agupubs.onlinelibrary.wiley.com/doi/abs/10.1002/ 2017JA024266 doi: 10.1002/2017JA024266

Poh, G., Slavin, J. A., Jia, X., Raines, J. M., Imber, S. M., Sun, W.-J., .. Smith, A. W. (2017b). Mercury's cross-tail current sheet: Structure, x-line location and stress balance. Geophysical Research Letters, 44(2), 678-686. Retrieved from https://agupubs.onlinelibrary.wiley.com/doi/abs/10.1002/ 2016GL071612 doi: 10.1002/2016GL071612

Pritchett, P. L., \& Coroniti, F. V. ～(2004). Three-dimensional collisionless magnetic reconnection in the presence of a guide field. Journal of Geophysical Research: Space Physics, 109(A1). Retrieved from https:// agupubs.onlinelibrary.wiley.com/doi/abs/10.1029/2003JA009999 doi: 10.1029/2003JA009999

Pulkkinen, T. I., Partamies, N., McPherron, R. L., Henderson, M., Reeves, G. D., Thomsen, M. F., \& Singer, H. J. (2007). Comparative statistical analysis of storm time activations and sawtooth events. Journal of Geophysical Research: Space Physics, 112(A1). Retrieved from https:// agupubs.onlinelibrary.wiley.com/doi/abs/10.1029/2006JA012024 doi: 10.1029/2006JA012024

Pytte, T., McPherron, R. L., Hones Jr., E. W., \& West Jr., H. I. (1978). Multiplesatellite studies of magnetospheric substorms: Distinction between polar magnetic substorms and convection-driven negative bays. Journal of Geophysical Research: Space Physics, 83(A2), 663-679. Retrieved from https:// agupubs.onlinelibrary.wiley.com/doi/abs/10.1029/JA083iA02p00663 doi: 10.1029/JA083iA02p00663

Raines, J. M., Gershman, D. J., Zurbuchen, T. H., Sarantos, M., Slavin, J. A., Gilbert, J. A., ... Solomon, S. C. (2013). Distribution and compositional variations of plasma ions in mercury's space environment: The first three mercury years of MESSENGER observations. Journal of Geophysical Research: Space Physics, 118(4), 1604-1619. Retrieved from https:// agupubs.onlinelibrary.wiley.com/doi/abs/10.1029/2012JA018073 doi: 10.1029/2012JA018073

Richardson, I. G., Cowley, S. W. H., Hones Jr., E. W., \& Bame, S. J. Plasmoid-associated energetic ion bursts in the deep geomagnetic tail: Properties of plasmoids and the postplasmoid plasma sheet. Journal of Geophysical Research: Space Physics, 92(A9), 9997-10013. Retrieved from https:// agupubs .onlinelibrary . wiley.com/doi/abs/10.1029/JA092iA09p09997 doi: 10.1029/JA092iA09p09997 
Rosenbauer, H., Grünwaldt, H., Montgomery, M. D., Paschmann, G., \& Sckopke, N. (1975). Heos 2 plasma observations in the distant polar magnetosphere: The plasma mantle. Journal of Geophysical Research (1896-1977), 80(19), 27232737. Retrieved from https://agupubs .onlinelibrary.wiley.com/doi/abs/ 10.1029/JA080i019p02723 doi: 10.1029/JA080i019p02723

Russell, C. T., Baker, D. N., \& Slavin, J. A. (1988). The magnetosphere of Mercury. In F. Vilas, C. R. Chapman, \& M. S. Matthews (Eds.), Mercury, university of arizona press (p. 514-561).

Sarantos, M., \& Slavin, J. A. (2009). On the possible formation of alfvén wings at mercury during encounters with coronal mass ejections. Geophysical Research Letters, 36(4). Retrieved from https://agupubs.onlinelibrary.wiley.com/ doi/abs/10.1029/2008GL036747 doi: 10.1029/2008GL036747

Sckopke, N., Paschmann, G., Rosenbauer, H., \& Fairfield, D. H. (1976). Influence of the interplanetary magnetic field on the occurrence and thickness of the plasma mantle. Journal of Geophysical Research (1896-1977), 81(16), 26872691. Retrieved from https://agupubs.onlinelibrary.wiley.com/doi/abs/ 10.1029/JA081i016p02687 doi: 10.1029/JA081i016p02687

Scurry, L., Russell, C. T., \& Gosling, J. T. (1994). Geomagnetic activity and the beta dependence of the dayside reconnection rate. Journal of Geophysical Research: Space Physics, 99(A8), 14811-14814. Retrieved from https://agupubs.onlinelibrary.wiley.com/doi/abs/10.1029/94JA00794 doi: 10.1029/94JA00794

Sergeev, V. A., Pellinen, R. J., \& Pulkkinen, T. I. (1996, Feb 01). Steady magnetospheric convection: A review of recent results. Space Science Reviews, 75(3), 551-604. Retrieved from https://doi.org/10.1007/BF00833344 doi: 10 $.1007 / \mathrm{BF} 00833344$

Shay, M. A., \& Swisdak, M. (2004, Oct). Three-species collisionless reconnection: Effect of $\mathrm{O}^{+}$on magnetotail reconnection. Phys. Rev. Lett., 93, 175001. Retrieved from https://link.aps.org/doi/10.1103/PhysRevLett.93.175001 doi: 10.1103/PhysRevLett.93.175001

Shue, J.-H., Song, P., Russell, C. T., Steinberg, J. T., Chao, J. K., Zastenker, G., ... Kawano, H. (1998). Magnetopause location under extreme solar wind conditions. Journal of Geophysical Research: Space Physics, 103(A8), 1769117700. Retrieved from https://agupubs.onlinelibrary.wiley.com/doi/ abs/10.1029/98JA01103 doi: 10.1029/98JA01103

Siscoe, G. L., Ness, N. F., \& Yeates, C. M. (1975). Substorms on mercury? Journal of Geophysical Research (1896-1977), 80(31), 4359-4363. Retrieved from https://agupubs.onlinelibrary.wiley.com/doi/abs/10.1029/ JA080i031p04359 doi: 10.1029/JA080i031p04359

Slavin, J. A., Acuña, M. H., Anderson, B. J., Baker, D. N., Benna, M., Boardsen, S. A., ... Zurbuchen, T. H. (2009). MESSENGER observations of magnetic reconnection in mercury's magnetosphere. Science, 324 (5927), 606-610. Retrieved from http://science.sciencemag.org/content/324/5927/606 doi: 10.1126/science.1172011

Slavin, J. A., Anderson, B. J., Baker, D. N., Benna, M., Boardsen, S. A., Gloeckler, G., ... Zurbuchen, T. H. (2010). MESSENGER observations of extreme loading and unloading of mercury's magnetic tail. Science, 329(5992), 665-668. Retrieved from http://science.sciencemag.org/content/329/5992/665 doi: 10.1126/science.1188067

Slavin, J. A., Anderson, B. J., Baker, D. N., Benna, M., Boardsen, S. A., Gold, R. E., ... Zurbuchen, T. H. (2012). MESSENGER and mariner 10 flyby observations of magnetotail structure and dynamics at mercury. Journal of Geophysical Research: Space Physics, 117(A1). Retrieved from https:// agupubs.onlinelibrary.wiley.com/doi/abs/10.1029/2011JA016900 doi: 10.1029/2011JA016900 
Slavin, J. A., DiBraccio, G. A., Gershman, D. J., Imber, S. M., Poh, G. K., Raines, J. M., ... Solomon, S. C. (2014). MESSENGER observations of mercury's dayside magnetosphere under extreme solar wind conditions. Journal of Geophysical Research: Space Physics, 119(10), 8087-8116. Retrieved from https://agupubs.onlinelibrary.wiley.com/doi/abs/10.1002/ 2014JA020319 doi: 10.1002/2014JA020319

Slavin, J. A., \& Holzer, R. E. (1979). The effect of erosion on the solar wind standoff distance at mercury. Journal of Geophysical Research: Space Physics, 84(A5), 2076-2082. Retrieved from https://agupubs.onlinelibrary.wiley .com/doi/abs/10.1029/JA084iA05p02076 doi: 10.1029/JA084iA05p02076

Slavin, J. A., Imber, S. M., Boardsen, S. A., DiBraccio, G. A., Sundberg, T., Sarantos, M., ... Solomon, S. C. (2012). MESSENGER observations of a fluxtransfer-event shower at mercury. Journal of Geophysical Research: Space Physics, 117(A12). Retrieved from https://agupubs.onlinelibrary.wiley .com/doi/abs/10.1029/2012JA017926 doi: 10.1029/2012JA017926

Slavin, J. A., Smith, M. F., Mazur, E. L., Baker, D. N., Hones Jr., E. W., Iyemori, T., \& Greenstadt, E. W.

Isee 3 observations of traveling compression regions in the earth's magnetotail. Journal of Geophysical Research: Space Physics, 98(A9), 15425-15446. Retrieved from https://agupubs.onlinelibrary.wiley.com/doi/abs/10.1029/93JA01467 doi: 10.1029/93JA01467

Slavin, J. A., Tanskanen, E. I., Hesse, M., Owen, C. J., Dunlop, M. W., Imber, S., ... Glassmeier, K.-H. (2005). Cluster observations of traveling compression regions in the near-tail. Journal of Geophysical Research: Space Physics, 110(A6). Retrieved from https://agupubs.onlinelibrary.wiley.com/doi/ abs/10.1029/2004JA010878 doi: 10.1029/2004JA010878

Smith, A. W., Jackman, C. M., Frohmaier, C. M., Fear, R. C., Slavin, J. A., \& Coxon, J. C. (2018). Evaluating single spacecraft observations of planetary magnetotails with simple monte carlo simulations: 2. magnetic flux rope signature selection effects. Journal of Geophysical Research: Space Physics, 123(12), 10,124-10,138. Retrieved from https://agupubs.onlinelibrary.wiley.com/ doi/abs/10.1029/2018JA025959 doi: 10.1029/2018JA025959

Smith, A. W., Slavin, J. A., Jackman, C. M., Poh, G.-K., \& Fear, R. C. (2017). Flux ropes in the hermean magnetotail: Distribution, properties, and formation. Journal of Geophysical Research: Space Physics, 122(8), 8136-8153. Retrieved from https://agupubs.onlinelibrary.wiley.com/doi/abs/ 10.1002/2017JA024295 doi: 10.1002/2017JA024295

Solomon, S. C., McNutt, R. L., Gold, R. E., Acuña, M. H., Baker, D. N., Boynton, W. V., ... Zuber, M. T. (2001). The MESSENGER mission to mercury: scientific objectives and implementation. Planetary and Space Science, 49(14), 1445 - 1465. Retrieved from http://www.sciencedirect.com/ science/article/pii/S003206330100085X (Returns to Mercury) doi: https://doi.org/10.1016/S0032-0633(01)00085-X

Sonnerup, B. U. O. (1974). Magnetopause reconnection rate. Journal of Geophysical Research (1896-1977), 79(10), 1546-1549. Retrieved from https:// agupubs . onlinelibrary. wiley.com/doi/abs/10.1029/JA079i010p01546 doi: 10.1029/JA079i010p01546

Sonnerup, B. U. O. (1979). Magnetic field reconnection. In L. J. Lanzerotti, C. F. Kennel, \& E. N. Parker (Eds.), Solar system plasma physics (p. 45-108).

Sonnerup, B. U. O., \& Cahill, L. J. (1967). Magnetopause structure and attitude from explorer 12 observations. Journal of Geophysical Research, 72(1), 171183. Retrieved from https://agupubs.onlinelibrary.wiley.com/doi/abs/ 10.1029/JZ072i001p00171 doi: 10.1029/JZ072i001p00171

Sonnerup, B. U. O., Paschmann, G., Papamastorakis, I., Sckopke, N., Haerendel, G., Bame, S. J., ... Russell, C. T. $\quad$ (1981). Evidence for magnetic 
field reconnection at the earth's magnetopause.

Journal of Geophysical Research: Space Physics, 86(A12), 10049-10067.

Retrieved from https:// agupubs . onlinelibrary .wiley.com/doi/abs/10.1029/JA086iA12p10049 doi: 10.1029/JA086iA12p10049

Sonnerup, B. U. O., \& Scheible, M. (1998). Minimum and maximum variance analysis. Analysis methods for multi-spacecraft data, 185-220.

Sun, W. J., Fu, S. Y., Parks, G. K., Liu, J., Yao, Z. H., Shi, Q. Q., .. Xiao, T. (2013). Field-aligned currents associated with dipolarization fronts. Geophysical Research Letters, 40(17), 4503-4508. Retrieved from https:// agupubs.onlinelibrary.wiley.com/doi/abs/10.1002/grl.50902 doi: $10.1002 /$ grl.50902

Sun, W. J., Fu, S. Y., Slavin, J. A., Raines, J. M., Zong, Q. G., Poh, G. K., \& Zurbuchen, T. H. (2016). Spatial distribution of mercury's flux ropes and reconnection fronts: MESSENGER observations. Journal of Geophysical Research: Space Physics, 121(8), 7590-7607. Retrieved from https:// agupubs.onlinelibrary.wiley.com/doi/abs/10.1002/2016JA022787 doi: 10.1002/2016JA022787

Sun, W. J., Raines, J. M., Fu, S. Y., Slavin, J. A., Wei, Y., Poh, G. K., ... Wan, W. X. (2017). MESSENGER observations of the energization and heating of protons in the near-mercury magnetotail. Geophysical Research Letters, 44(16), 8149-8158. Retrieved from https://agupubs.onlinelibrary.wiley .com/doi/abs/10.1002/2017GL074276 doi: 10.1002/2017GL074276

Sun, W. J., Slavin, J. A., Dewey, R. M., Raines, J. M., Fu, S. Y., Wei, Y., ... Zhao, D. (2018). A comparative study of the proton properties of magnetospheric substorms at earth and mercury in the near magnetotail. Geophysical Research Letters, $O(0)$. Retrieved from https://agupubs.onlinelibrary.wiley.com/ doi/abs/10.1029/2018GL079181 doi: 10.1029/2018GL079181

Sun, W.-J., Slavin, J. A., Fu, S., Raines, J. M., Zong, Q.-G., Imber, S. M., ... Baker, D. N. (2015). MESSENGER observations of magnetospheric substorm activity in mercury's near magnetotail. Geophysical Research Letters, 42(10), 3692-3699. Retrieved from https://agupubs.onlinelibrary.wiley.com/ doi/abs/10.1002/2015GL064052 doi: 10.1002/2015GL064052

Sun, W. J., Slavin, J. A., Tian, A. M., Bai, S. C., Poh, G. K., Akhavan-Tafti, M., ... Burch, J. L. (2019). Mms study of the structure of ion-scale flux ropes in the earth's cross-tail current sheet. Geophysical Research Letters, 46(12), 6168-6177. Retrieved from https://agupubs.onlinelibrary.wiley.com/ doi/abs/10.1029/2019GL083301 doi: 10.1029/2019GL083301

Sundberg, T., Slavin, J. A., Boardsen, S. A., Anderson, B. J., Korth, H., Ho, G. C., ... Solomon, S. C. (2012). MESSENGER observations of dipolarization events in mercury's magnetotail. Journal of Geophysical Research: Space Physics, 117(A12). Retrieved from https://agupubs.onlinelibrary.wiley.com/ doi/abs/10.1029/2012JA017756 doi: 10.1029/2012JA017756

Tanskanen, E. I., Slavin, J. A., Fairfield, D. H., Sibeck, D. G., Gjerloev, J., Mukai, T.,... Nagai, T. (2005). Magnetotail response to prolonged southward imf bz intervals: Loading, unloading, and continuous magnetospheric dissipation. Journal of Geophysical Research: Space Physics, 110(A3). Retrieved from https://agupubs.onlinelibrary.wiley.com/doi/abs/10.1029/ 2004JA010561 doi: 10.1029/2004JA010561

Winslow, R. M., Anderson, B. J., Johnson, C. L., Slavin, J. A., Korth, H., Purucker, M. E., ... Solomon, S. C. (2013). Mercury's magnetopause and bow shock from MESSENGER magnetometer observations. Journal of Geophysical Research: Space Physics, 118(5), 2213-2227. Retrieved from https://agupubs.onlinelibrary.wiley.com/doi/abs/10.1002/jgra. 50237 doi: $10.1002 /$ jgra. 50237

Winslow, R. M., Lugaz, N., Philpott, L. C., Schwadron, N. A., Farrugia, C. J., 
Anderson, B. J., \& Smith, C. W. (2015). Interplanetary coronal mass ejections from messenger orbital observations at mercury. Journal of Geophysical Research: Space Physics, 120(8), 6101-6118. Retrieved from https:// agupubs.onlinelibrary. wiley.com/doi/abs/10.1002/2015JA021200 doi: 10.1002/2015JA021200

Yang, J., Toffoletto, F. R., \& Song, Y. (2010). Role of depleted flux tubes in steady magnetospheric convection: Results of rcm-e simulations. Journal of Geophysical Research: Space Physics, 115(A5). Retrieved from https:// agupubs .onlinelibrary.wiley.com/doi/abs/10.1029/2010JA015731 doi: 10.1029/2010JA015731

Zhang, B., Brambles, O. J., Lotko, W., Ouellette, J. E., \& Lyon, J. G. (2016). The role of ionospheric o+ outflow in the generation of earthward propagating plasmoids. Journal of Geophysical Research: Space Physics, 121(2), 14251435. Retrieved from https://agupubs.onlinelibrary.wiley.com/doi/abs/ 10.1002/2015JA021667 doi: 10.1002/2015JA021667

Zhao, J. T., Sun, W. J., Zong, Q. G., Slavin, J. A., Zhou, X. Z., Dewey, R. M., ... Raines, J. M. (2019). A statistical study of the force balance and structure in the flux ropes in mercury's magnetotail. Journal of Geophysical Research: Space Physics, O(ja). Retrieved from https://agupubs.onlinelibrary .wiley.com/doi/abs/10.1029/2018JA026329 doi: 10.1029/2018JA026329

Zhong, J., Wei, Y., Pu, Z. Y., Wang, X. G., Wan, W. X., Slavin, J. A., ... Li, Y. (2018). MESSENGER observations of rapid and impulsive magnetic reconnection in mercury's magnetotail. The Astrophysical Journal Letters, 860(2), L20. Retrieved from http://stacks.iop.org/2041-8205/860/i=2/a=L20

Zong, Q.-G., Fritz, T. A., Pu, Z. Y., Fu, S. Y., Baker, D. N., Zhang, H., .. Reme, H. (2004). Cluster observations of earthward flowing plasmoid in the tail. Geophysical Research Letters, 31(18). Retrieved from https:// agupubs.onlinelibrary.wiley.com/doi/abs/10.1029/2004GL020692 doi: 10.1029/2004GL020692 
Figure2_23Nov2011_V01.pdf.

This article is protected by copyright. All rights reserved. 
MESSENGER MAG FIPS 2011 DOY 327 (23-Nov)

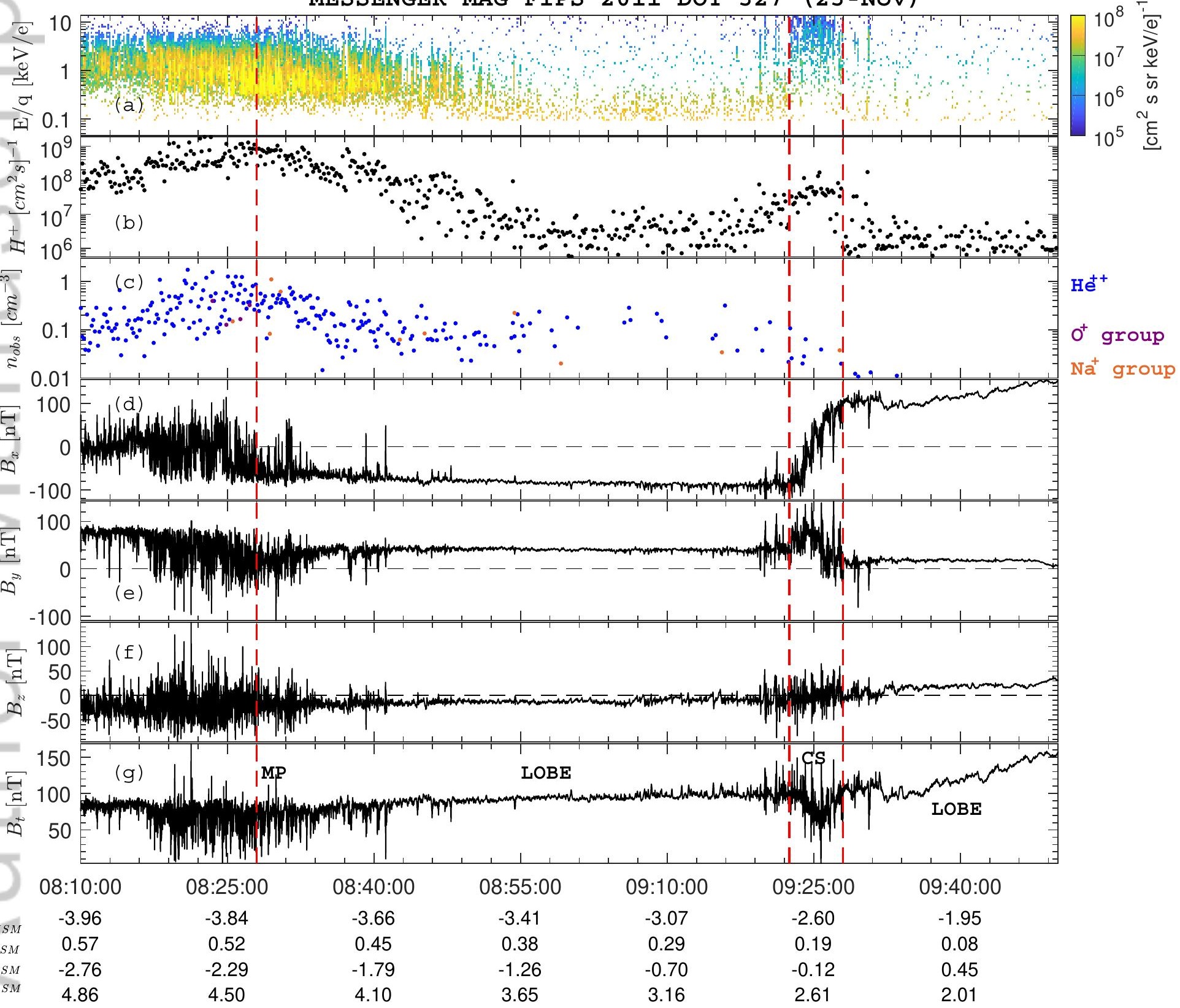


Figure3_11May2012_V01.pdf.

This article is protected by copyright. All rights reserved. 
MESSENGER MAG FIPS 2012 DOY 132 (11-May) 
Figure4_23Nov2011_V01.pdf.

This article is protected by copyright. All rights reserved. 
MESSENGER MAG FIPS 2011 DOY 327 (23-Nov)

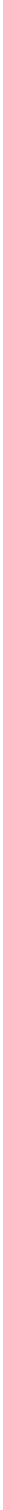


Figure5_quasiperiod_fluxropes_23Nov2011_v01.pdf.

This article is protected by copyright. All rights reserved. 


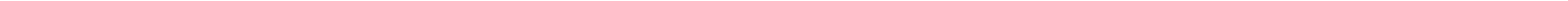


Figure6_Scatter_FRTCRs_BNBTDuration_V03.pdf.

This article is protected by copyright. All rights reserved. 
Figure7_PlasmaSheet_11May2012_V01.pdf.

This article is protected by copyright. All rights reserved. 
MESSENGER MAG FIPS 2012 DOY $132,(11-$ May)

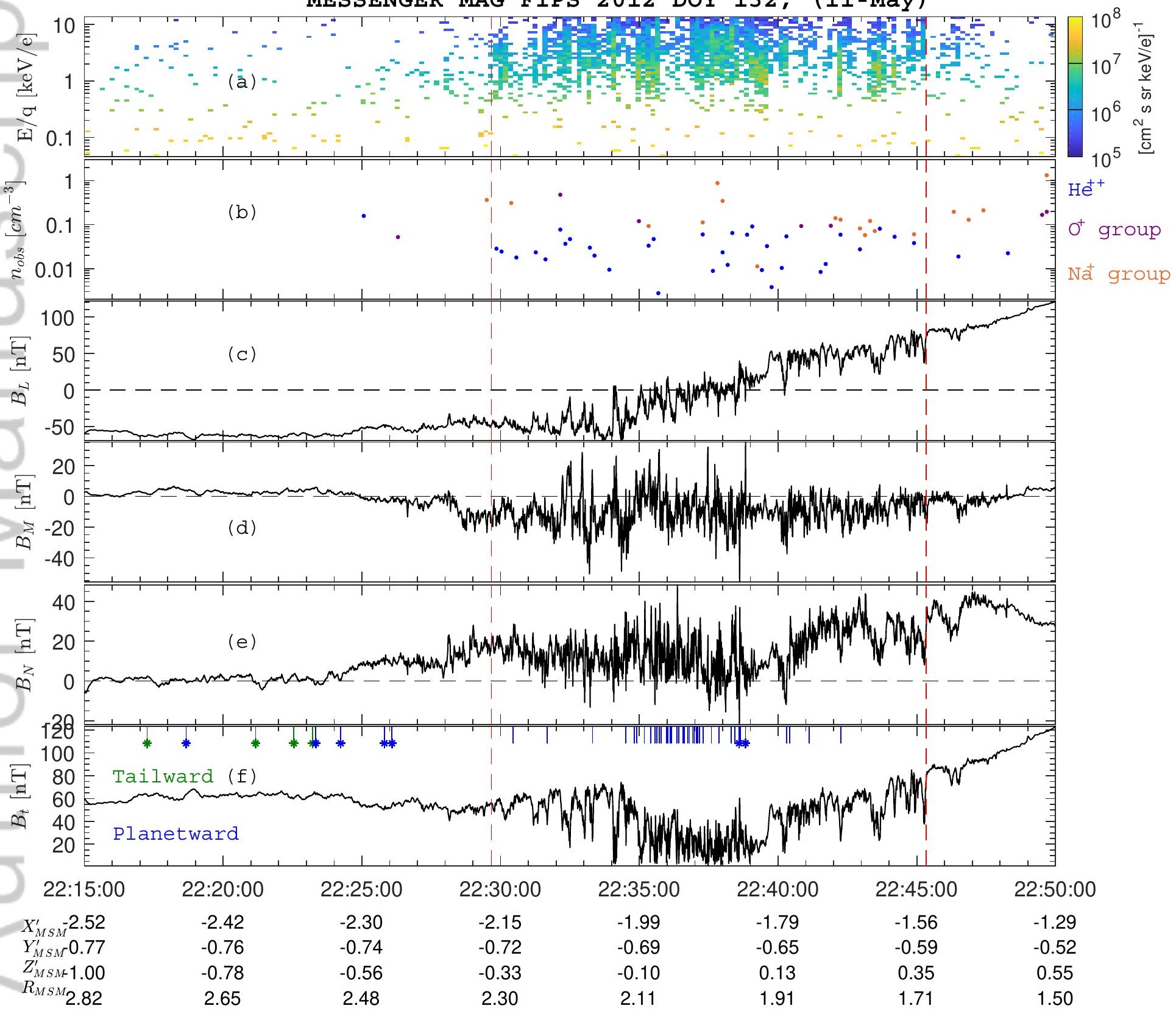


Figure8_RFs_11May2012_V01.pdf.

This article is protected by copyright. All rights reserved. 


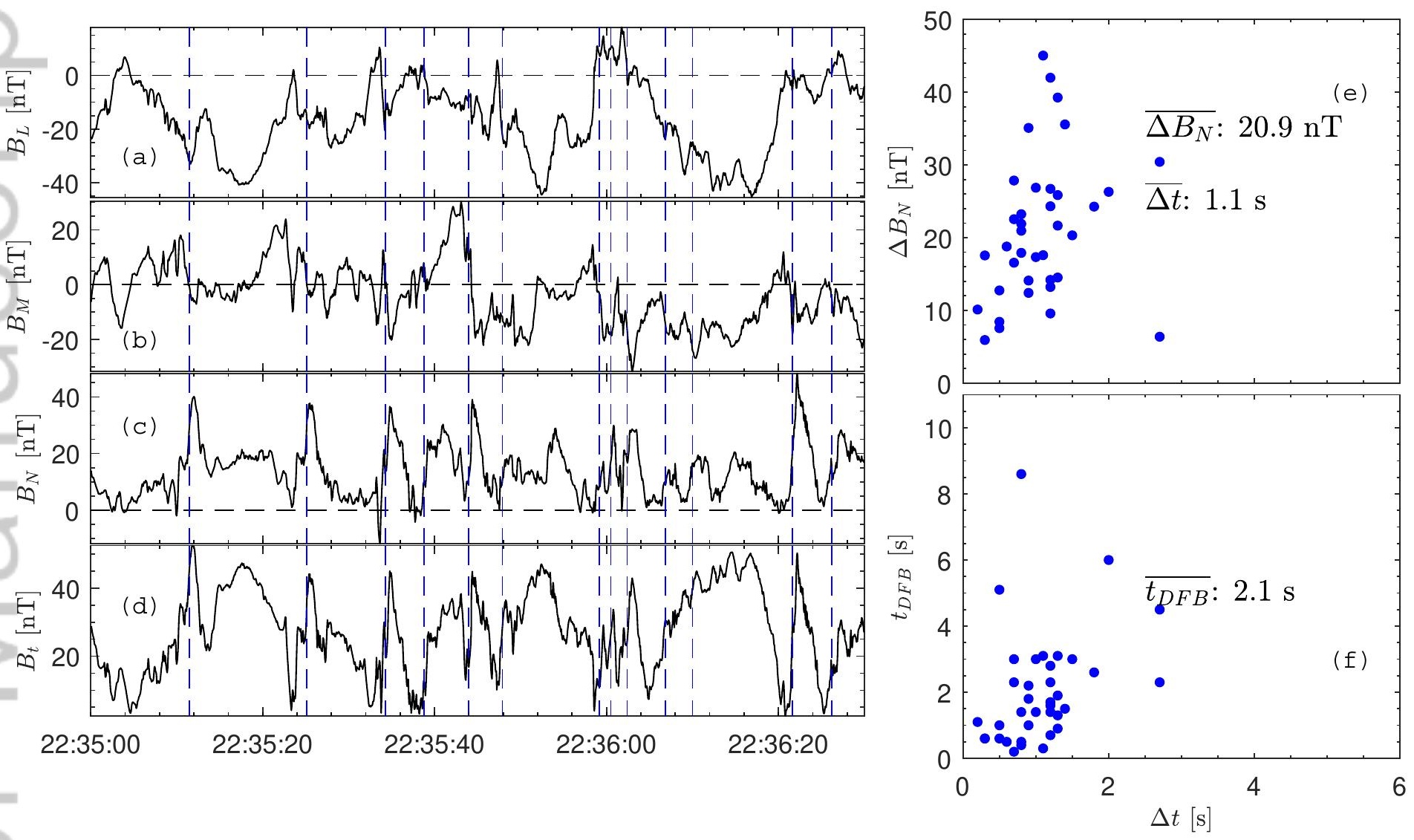


Figure9_HallB_Field.pdf.

This article is protected by copyright. All rights reserved. 


\section{DOY 327 (23-Nov)}

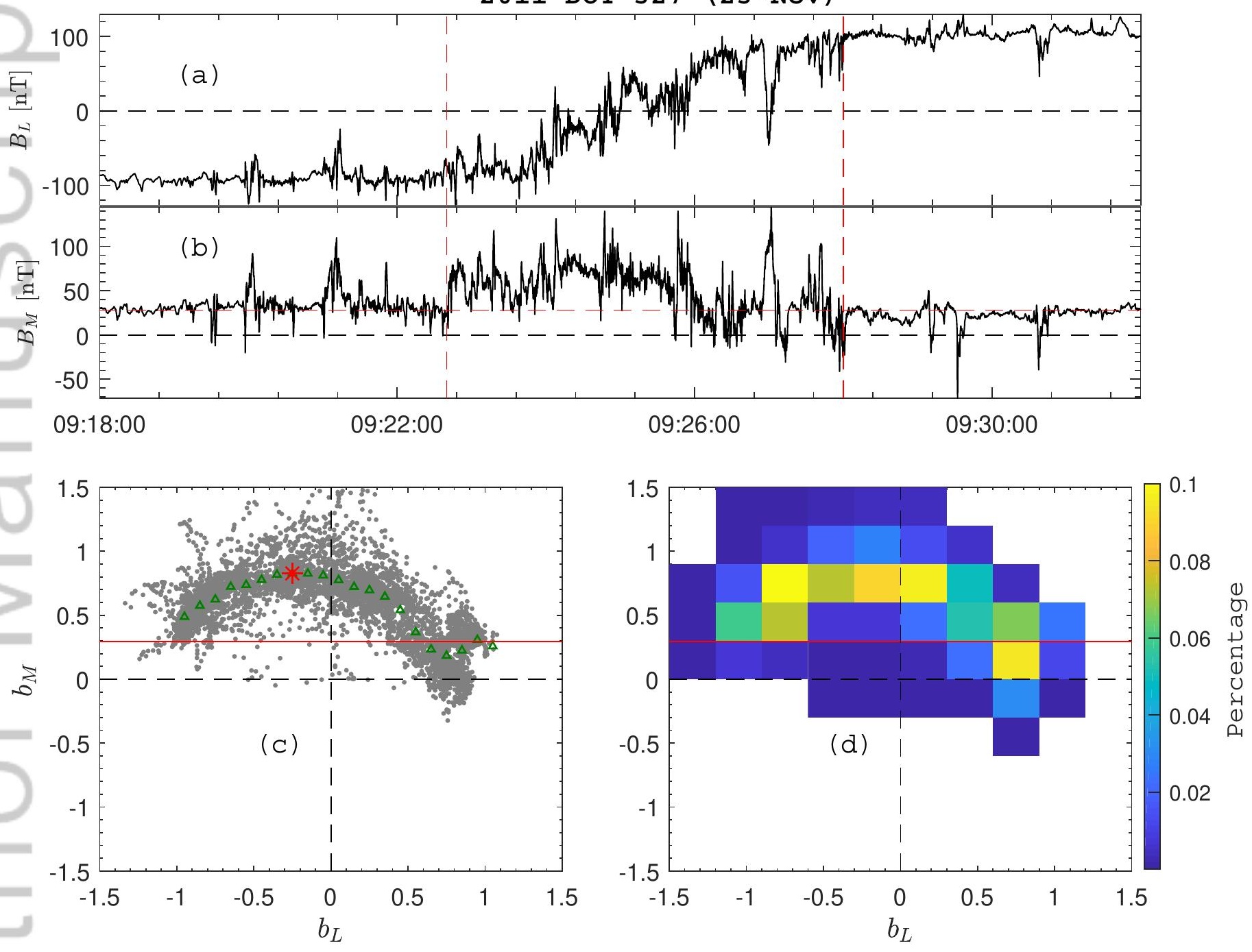


Figure10_HallB_Field.pdf.

This article is protected by copyright. All rights reserved. 
(a)

\section{Guide Field}

$\bullet$
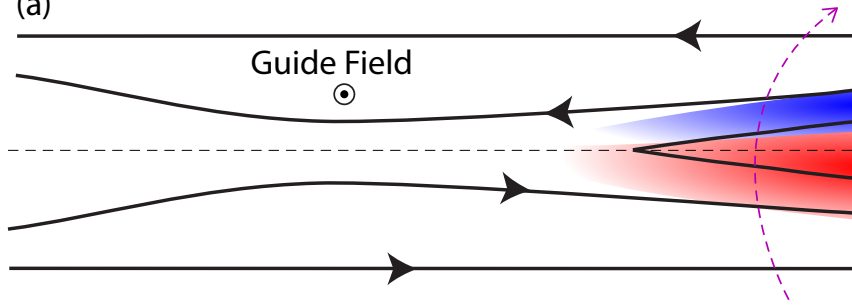

(b) 
Figure11_HCS_Modeling_BN_V01.pdf.

This article is protected by copyright. All rights reserved. 

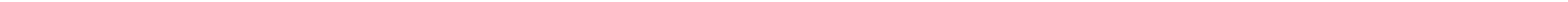
Figure12_Lobe_BField.pdf.
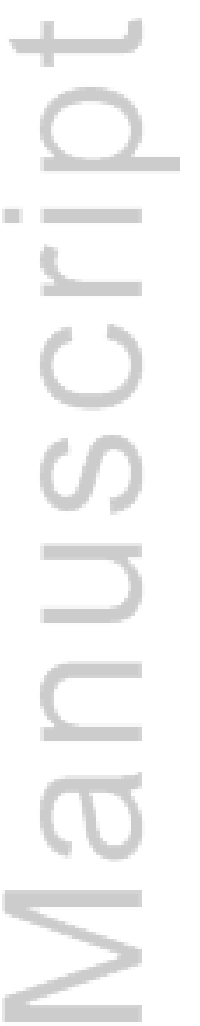

This article is protected by copyright. All rights reserved. 
2011 DOY 327 (23-Nov)

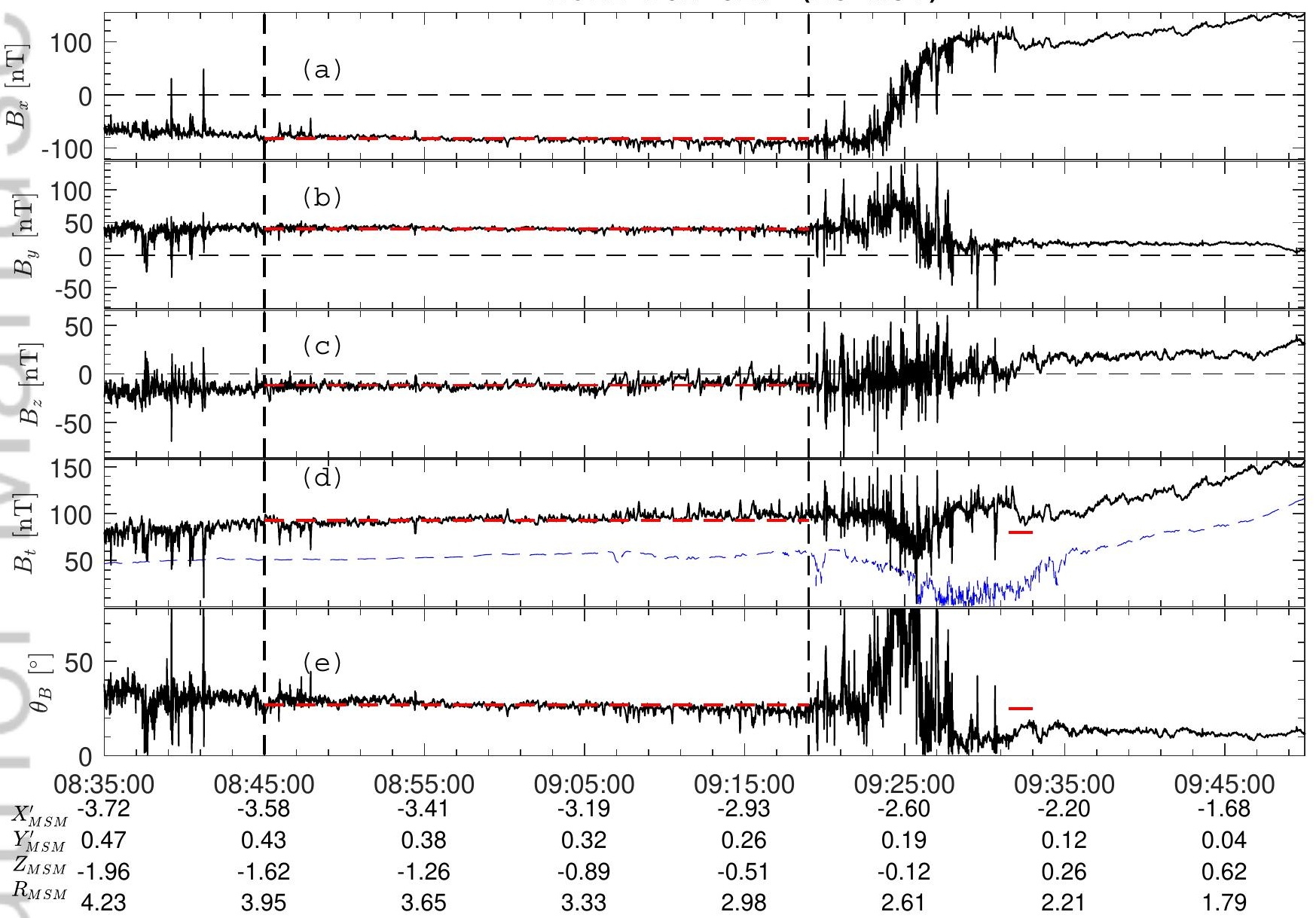


Figure13_Lobe_BField_11May2012.pdf.

This article is protected by copyright. All rights reserved. 


\section{DOY 132 (11-May)}

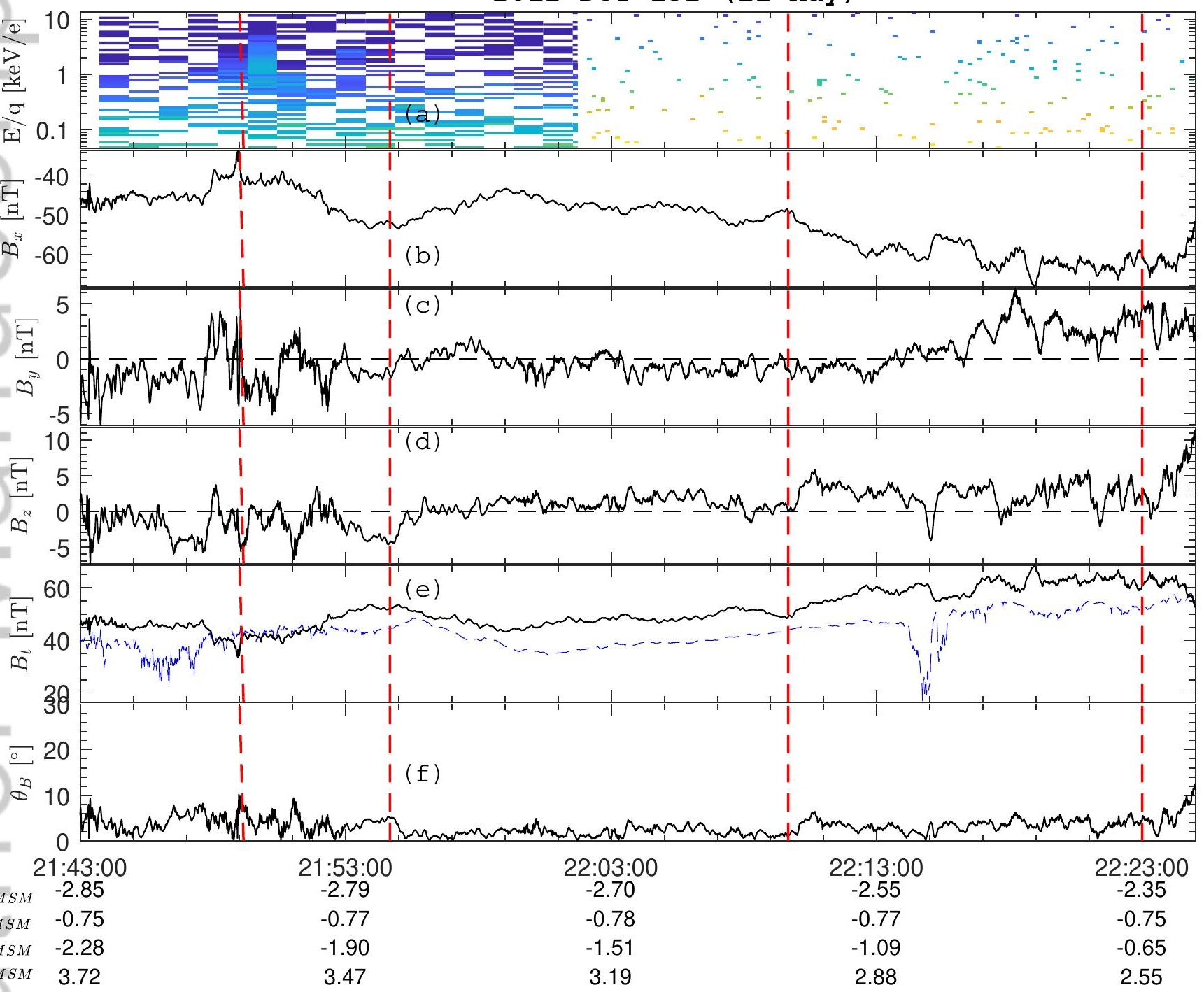


Figure14_Mantle_V_theta_Veb_Potential_V01.pdf.

This article is protected by copyright. All rights reserved. 
Figure15_Mantle.pdf.
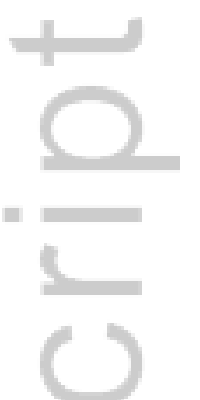

$(0$
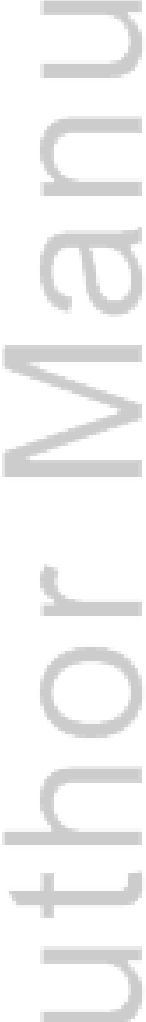

This article is protected by copyright. All rights reserved. 

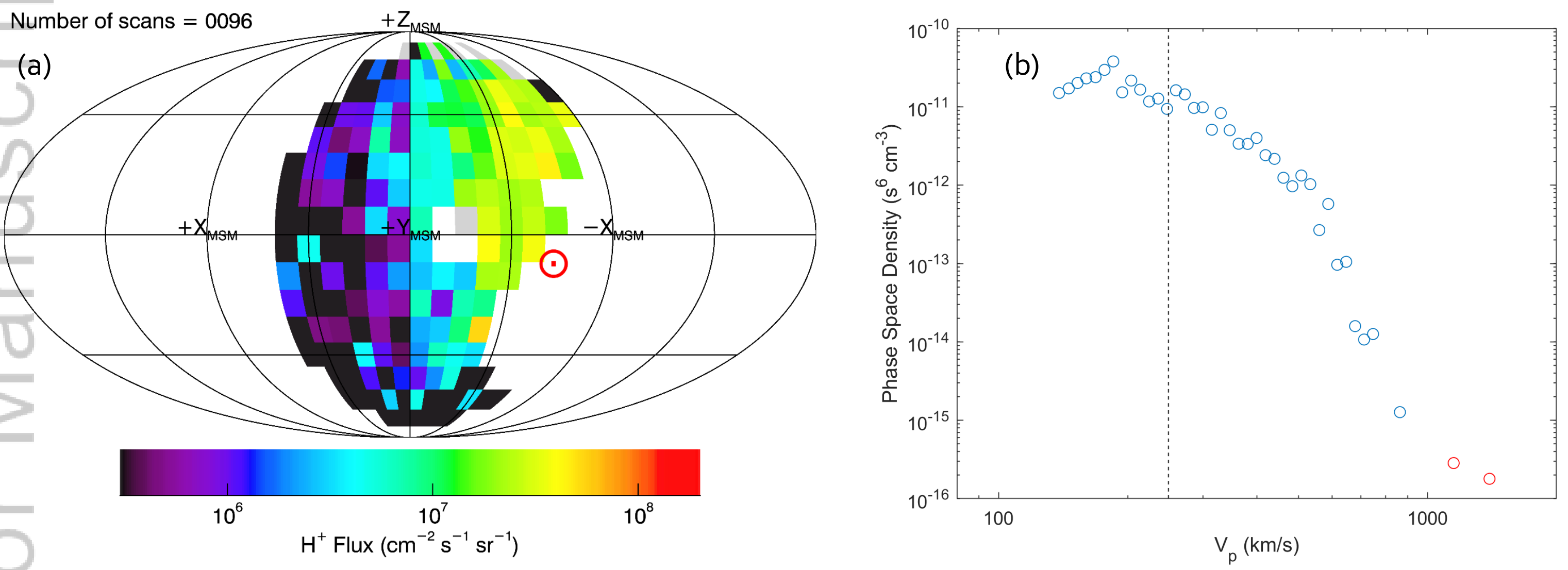
Table 2. Features of Steady Convection events at Mercury and Earth

Steady Convection Events at Mercury Solar Wind Condition

6 October $2008^{\mathrm{b}}$

Average Solar Wind intensity

NMNL Location

Lobe Open Flux Comparing to Average Lobe Open Flux $(\sim 2.5 \mathrm{MWb})^{\mathrm{a}}$

23 November 2011

A CME

$\sim-2.8 \mathrm{R}_{\mathrm{M}}$

$\sim 2.46 \mathrm{MWb} \sim-1.6 \%$

11 May2012

An HSS

$\sim-2.51 \mathrm{R}_{\mathrm{M}}$ to $\sim-2.28 \mathrm{R}_{\mathrm{M}} \sim 4.2 \mathrm{MWb}$

$\sim 68 \%$

SMC Events at Earth ${ }^{c}$

Solar Wind Condition $\quad$ NENL Location $^{\mathrm{d}}$

$\begin{array}{llll}\text { Solar Wind Condition } & \text { NENL Location }^{\mathrm{d}} & \text { Lobe Open Flux } & \text { Comp } \\ \text { Average Solar Wind intensity } & <-60 \mathrm{R}_{\mathrm{E}}\left(\sim 7.5 \mathrm{R}_{\mathrm{M}}\right) & \sim 0.6 \mathrm{GWb} & \sim 0 \%\end{array}$

$\sim 3.2 \mathrm{MWb}$

$\sim 27.2 \%$

${ }^{a}$ Average lobe open flux at Mercury is $2.5 \mathrm{MWb}$ (Imber \& Slavin, 2017), and at Earth is $\sim 0.6 \mathrm{GWb}$ (Milan et al., 2004)

${ }^{b}$ This event was from Slavin, Anderson, et al. (2012).

${ }^{c}$ The statistical properties of SMC at Earth are from DeJong et al. (2007); O'Brien et al. (2002); Yang et al. (2010) etc.

${ }^{\mathrm{d}}$ The scaling factor from Mercury to Earth is 8 (Siscoe et al., 1975).

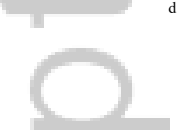


MESSENGER MAG FIPS 2011 DOY 327 (23-Nov)

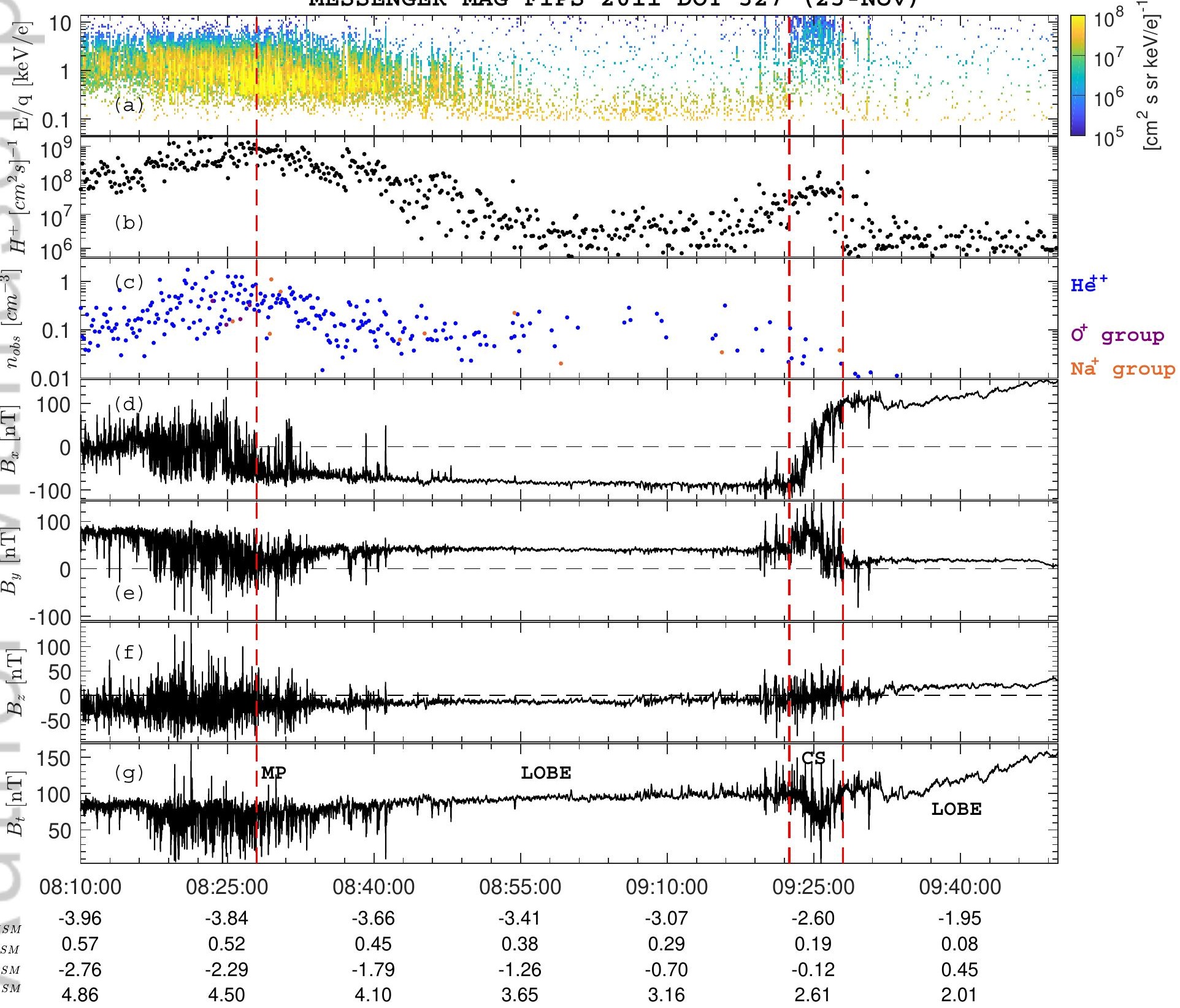


MESSENGER MAG FIPS 2012 DOY 132 (11-May) 
MESSENGER MAG FIPS 2011 DOY 327 (23-Nov)

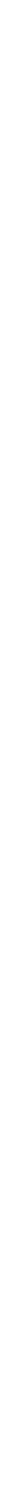




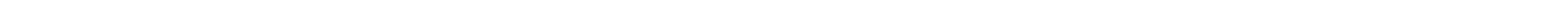


MESSENGER MAG FIPS 2012 DOY $132,(11-$ May)

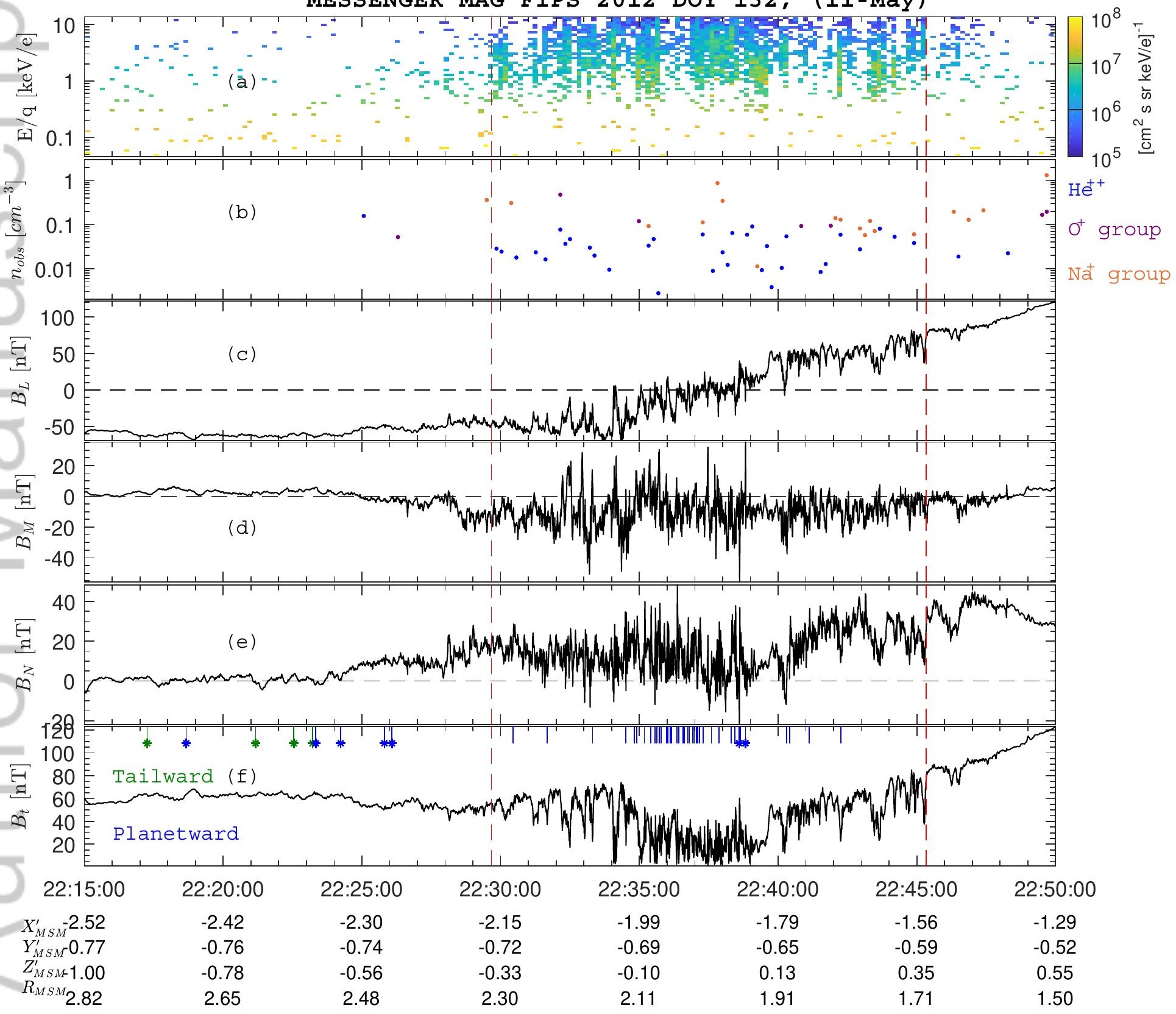




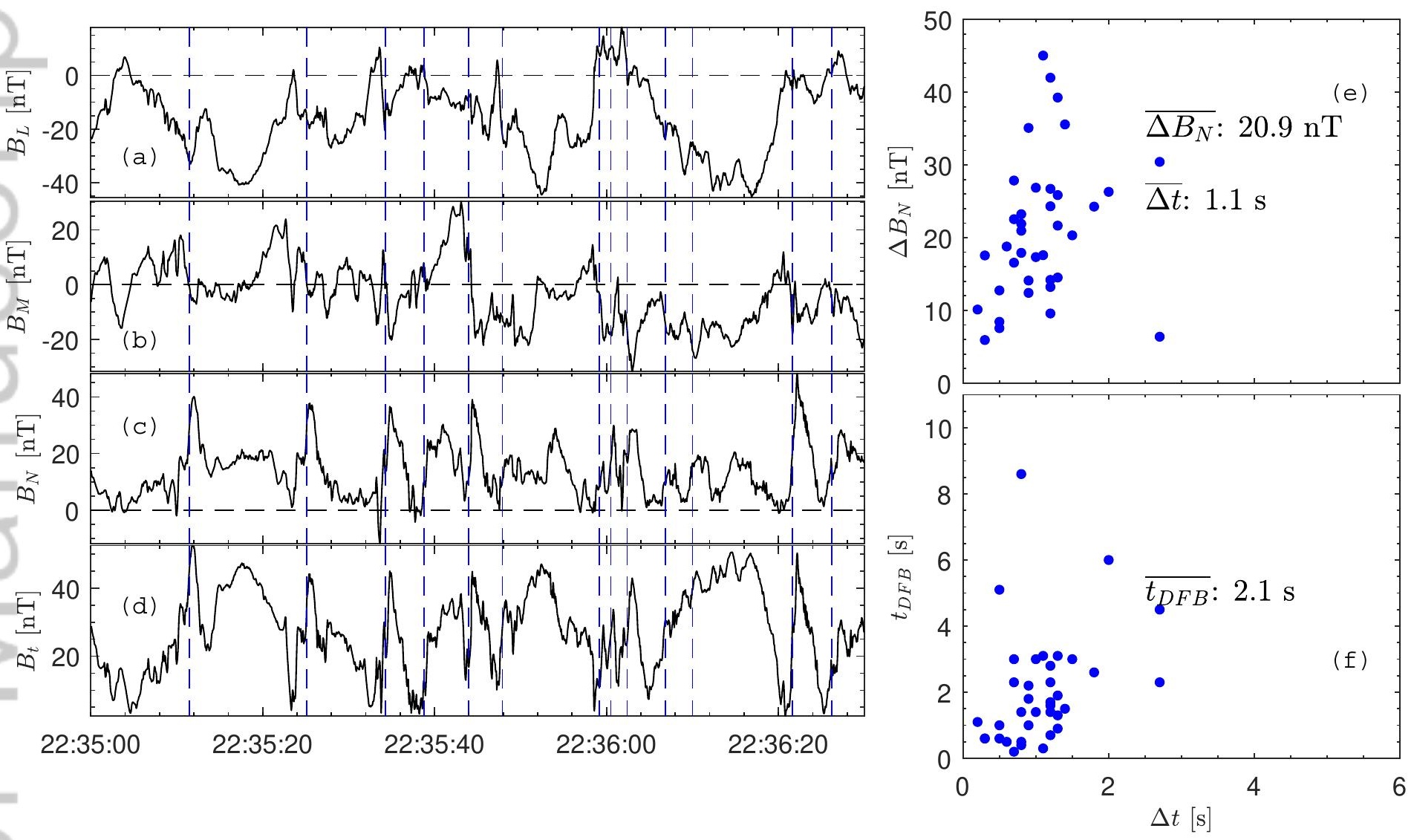




\section{DOY 327 (23-Nov)}

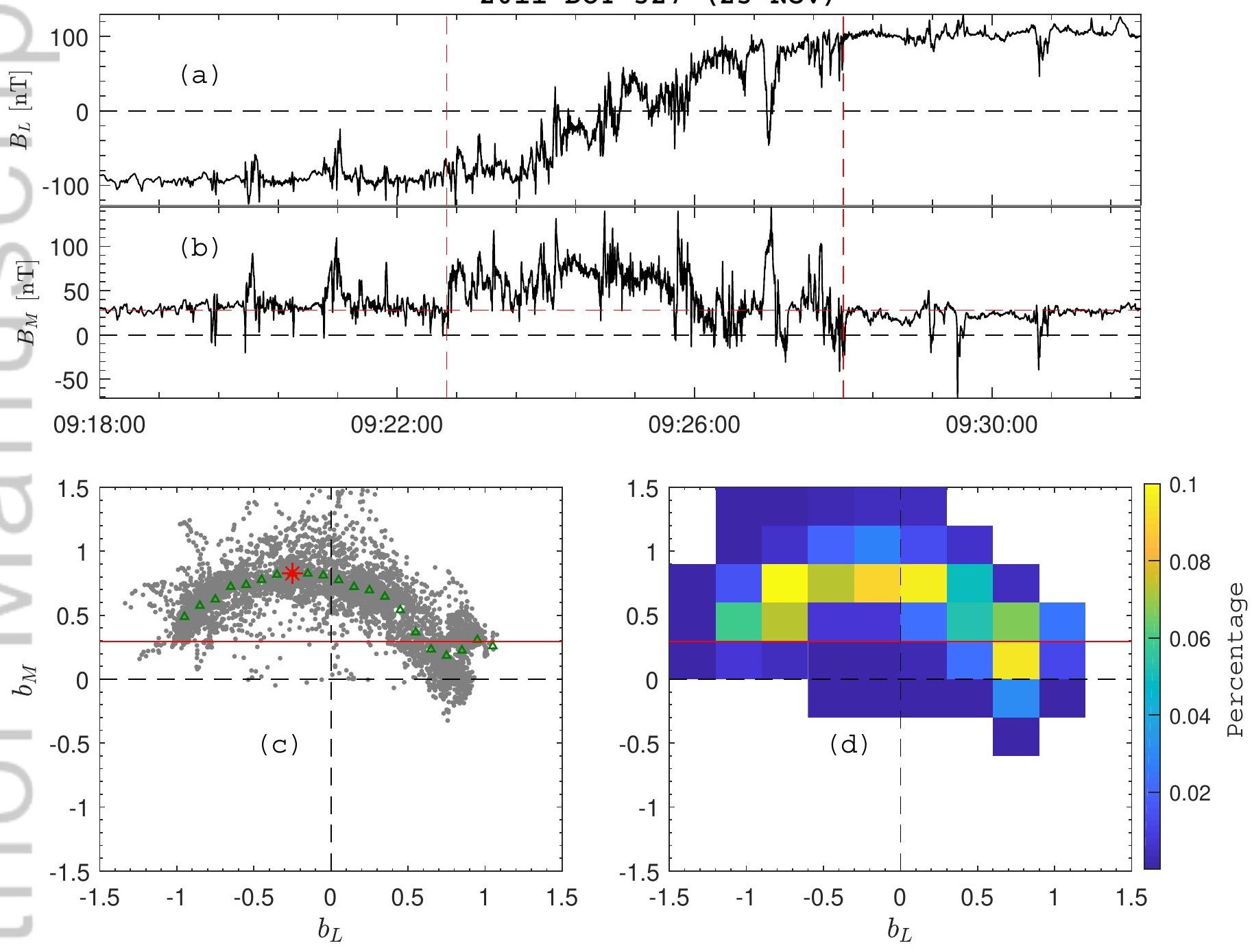


(a)

\section{Guide Field}

$\bullet$
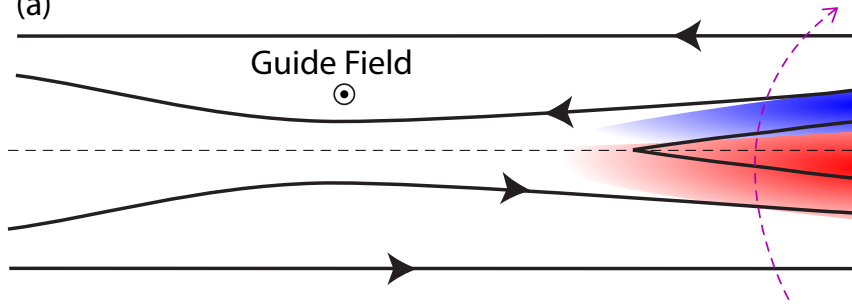

(b) 

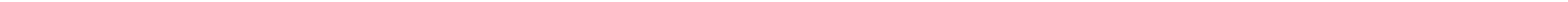
2011 DOY 327 (23-Nov)

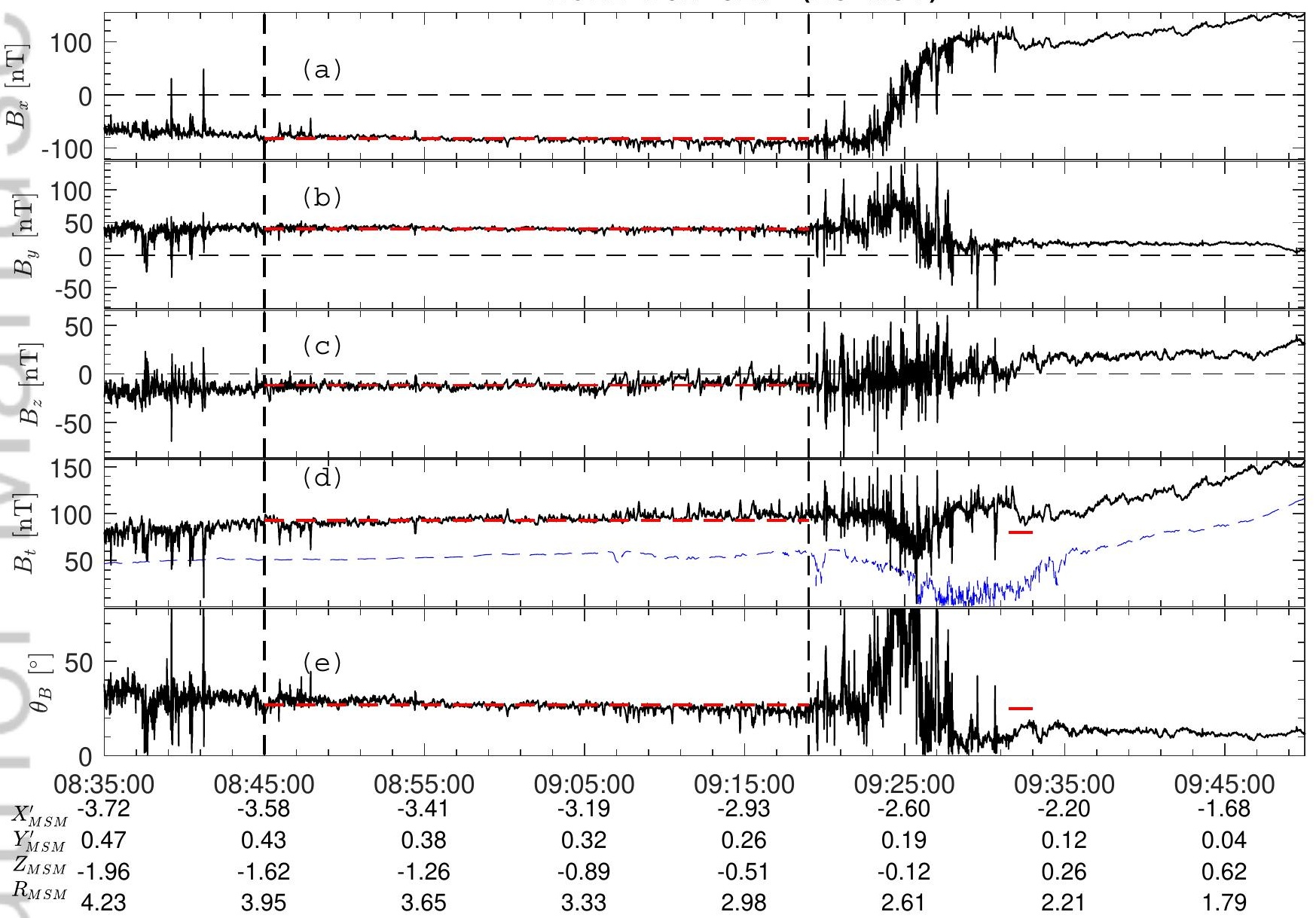




\section{DOY 132 (11-May)}

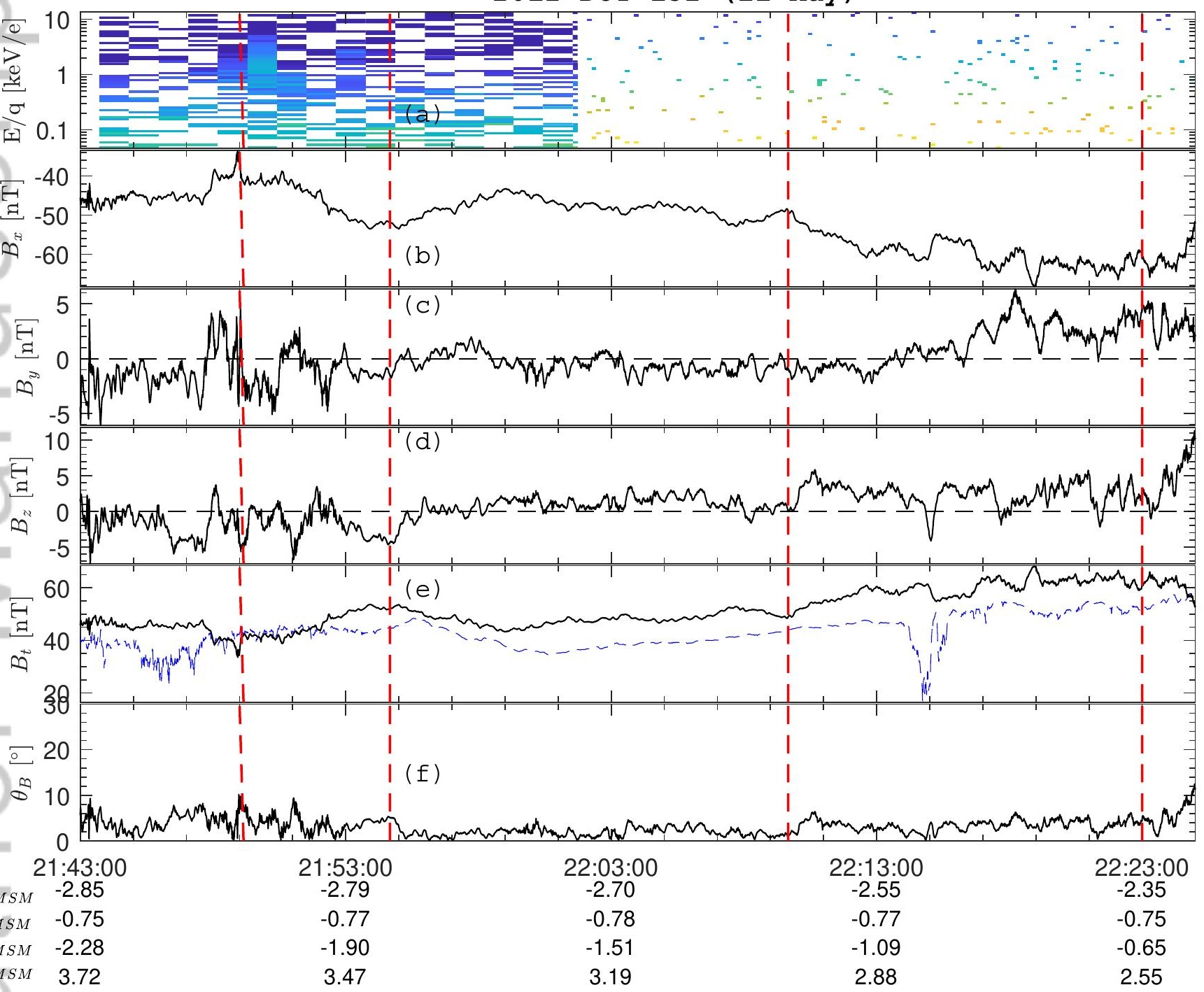



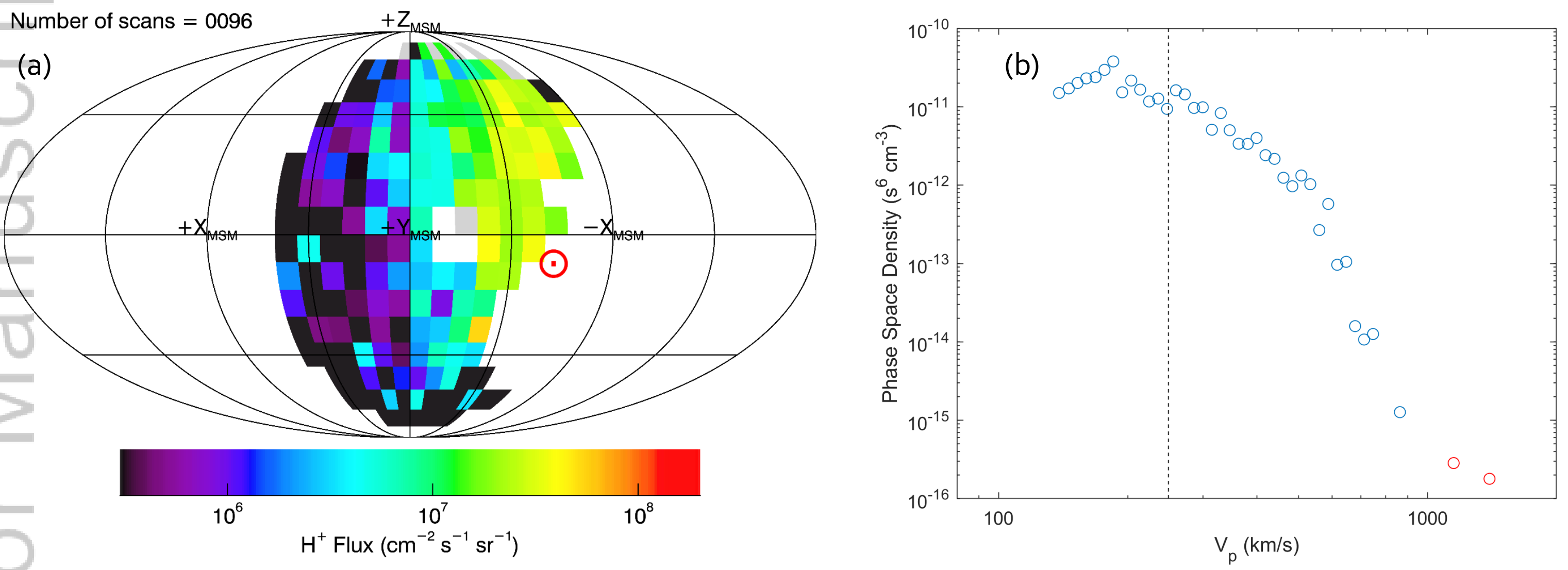